School of Geography, Environment, and Earth Sciences

Victoria University of Wellington

New Zealand

\title{
The influence of culture on ethnic women's use of microfinance for empowerment in Luang Namtha Province, Laos
}

\section{Souasong Tongnamavong}

A thesis submitted to Victoria University of Wellington in partial fulfilment of the requirements for the degree of Master of

Development Studies 



\section{Abstract}

The empowerment of women is essential but remains challenging globally. It has been supported by microfinance projects, particularly in recent decades across poor countries. Yet the contribution of microfinance to women's empowerment remains controversial given the complexity of empowerment itself. It is commonly acknowledged that empowerment starts from within individuals themselves. It is also acknowledged that culture plays a significant role in women's empowerment. Despite these acknowledgements, the complexities remain unclear in the Lao context which is the target of this study.

This study examines the contribution of microfinance to the empowerment of women in Laos with a focus on the elements of culture. It is based on a feminist perspective, with a focus on a personal empowerment conceptual framework which enables the core values of empowerment to be examined. The examination is undertaken by employing semistructured interviews and focus groups for data collection with three ethnic groups in northern Laos, namely Hmong, Khmu and Tai-Yuan, participating in the Access to Finance for the Poor (AFP) project.

The results show that the approach of the AFP project tends to meet women's practical needs rather than their strategic needs. This is because the project's approach appears to be influenced by the national approach of Laos in which the provision of opportunities for women is emphasised. It is also because ethnic women's participation in the project is driven by so many practical needs that it makes their strategic needs invisible. The results also revealed that the women's strategic needs are diverse, due to their particular culture and traditions. The results showed several gaps in the empowerment of women in the country. If these gaps are to be closed, there is a need for efforts from many stakeholders, particularly in the education sector, as the gender agenda needs to be integrated into its national curriculum.

Keywords: women's empowerment, microfinance, village banks, culture, Laos 
This page is intentionally left blank. 


\section{Acknowledgments}

This thesis is a result of the contributions from several organisations and people who I would like to acknowledge and say thank you to from the bottom of my heart in a formal to informal order.

I would like to firstly thank the Ministry of Foreign Affairs and Trade of New Zealand for generously providing me with the scholarship which has helped sustain my academic study through both the coursework and thesis years. Without your financial support, this thesis would not exist.

Thank you Victoria International, particularly Tim Lawther, who regularly encouraged me in each academic monitoring meeting to study better. Your care and attention were inspiring. Each individual meeting with you echoed the belief that I can be successful. Clemmie Newton, despite your new appointment at Victoria International, I am grateful that you helped arrange a proofreader for me successfully.

Thank you Student Learning of Victoria University of Wellington (VUW) for your excellent academic support, particularly during my coursework study. Your great staff members helped me through daunting assignments, providing guidance on essay structure, argument, and language polishing, during the beginning of my study at VUW in particular.

I am particularly indebted to Prof. John Overton, my supervisor, for your effort and excellent advice which made this research possible, smooth, and kept it on track. I feel that saying thank you is never enough to express my gratitude toward your contribution to my academic success. My confidence through the research process was maintained due to your encouragement and positive attitude. I usually felt optimistic after each meeting with you. In addition to academic achievements, you helped increase my self-confidence, independency, and optimistic attitude. My very first class with you already created echoes of my inspiration for success. Without you, I may have been through more suffering and hurdles.

The fieldwork of this research was enabled with support from the Bank of Laos, particularly the Financial Institutions Supervision Department, and Deutsche Gesellschaft für Internationale Zusammenarbeit, GmbH (GIZ). Your hearty and friendly support made me feel so welcomed. Your great staff from central to local offices, particularly GIZ office in Luang Namtha province, were very supportive. A simple thank you is never enough for all the help all of you gave me throughout the fieldwork. 
Rohini Biradavolu, my librarian: I want to thank you for assisting me through several writing assignments. Your help regarding citation and references means a lot to me. I sometimes accidentally approached you without making any appointment in advance, but you were so welcoming and were willing to sit with me, be informative, and supportive. I was amazed with your ability to help solve some of my technical computer problems. That was amazing, and I hope you were surprised too.

My general thank you is for any organisations and individuals not mentioned in this acknowledgement. I understand that your support and participation in this research are equally important.

Finally, I cannot forget to thank my family, particularly my brothers and sisters at home who helped sustain the family's wellbeing and happiness during my absence. I know I cannot give up because all of you are waiting for me at home. You were so encouraging any time I felt like giving up.

Wellington, 04 March 2019

Souasong Tongnamavong

Master in Development Studies

Victoria University of Wellington

New Zealand 
Acronyms

\begin{tabular}{|c|c|}
\hline $\mathrm{ADB}$ & Asian Development Bank \\
\hline AFP & Access to Finance for the Poor \\
\hline ASEAN & Association of Southeast Asian Nations \\
\hline $\mathrm{BoL}$ & Bank of Laos \\
\hline BRAC & Bangladesh Rural Advancement Committee \\
\hline CEDAW & Committee on the Elimination of Discrimination against Women \\
\hline CLFNC & Committee of Lao Front for National Construction \\
\hline DFAT-AG & $\begin{array}{l}\text { Department of Foreign Affairs and Trade of the Australian } \\
\text { Government }\end{array}$ \\
\hline DMVB & Donmoun Village Bank \\
\hline FAO & Food and Agriculture Organisation \\
\hline FISD-BoL & Financial Institution Supervision Department of Bank of Laos \\
\hline GAD & Gender and Development \\
\hline GDP & Gross Domestic Product \\
\hline GIZ Laos-AFP & $\begin{array}{l}\text { Access to Finance for the Poor project of Deutsche Gesellschaft } \\
\text { für Internationale Zusammenarbeit, GmbH (or German Society } \\
\text { for International Cooperation, Ltd.) in Laos }\end{array}$ \\
\hline HDI & Human Development Index \\
\hline JICA & Japan International Cooperation Agency \\
\hline Lao PDR & Lao People's Democratic Republic \\
\hline LDC & Least Developed Country \\
\hline LFNC & Lao Front for National Construction \\
\hline LNTD & Luang Namtha Tourism Department \\
\hline LPRP & Lao People's Revolutionary Party \\
\hline LWU & Lao Women's Union \\
\hline MDGs & Millennium Development Goals \\
\hline MoNRE & Ministry of Natural Resources and Environment \\
\hline MPI & Ministry of Planning and Investment \\
\hline NCAW & National Committee for the Advancement of Women \\
\hline NDTMFIs & Non-Deposit-Taking Microfinance Institutions \\
\hline NDTVB & Namdaeng Tai Village Bank \\
\hline NEM & New Economic Mechanism \\
\hline NGOs & Non-Governmental Organisations \\
\hline NSEDP & National Socio-Economic Development Plan \\
\hline NSOs & Network Support Organisations \\
\hline PAR & Portfolio At Risk \\
\hline
\end{tabular}


SDGs

SHG

SMEs

UN

UNDP

VB

VBC

VNVB

VUW

VUW-HEC

WAD

WID
Sustainable Development Goals

Self-Help Group

Small and Medium Enterprises

United Nations

United Nations Development Programme

Village Bank

Village Bank Committee

Viengnuea Village Bank

Victoria University of Wellington

Human Ethics Committee of Victoria University of Wellington

Women and Development

Women in Development 


\section{Table of Contents}

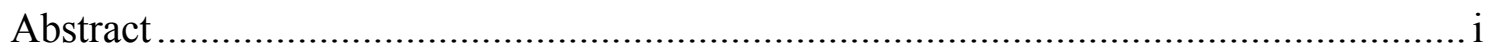

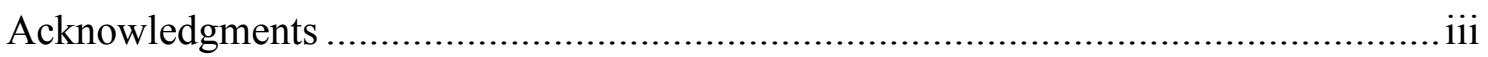

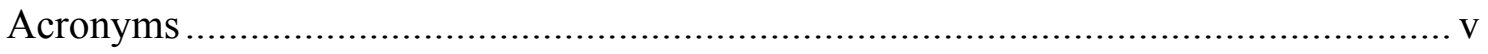

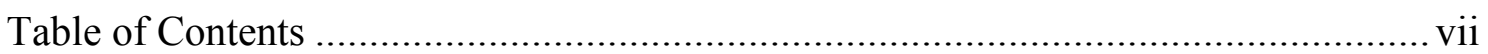

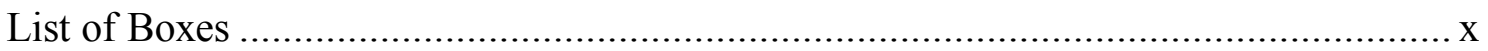

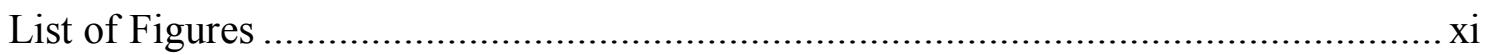

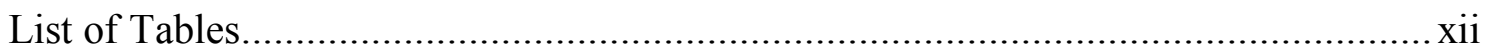

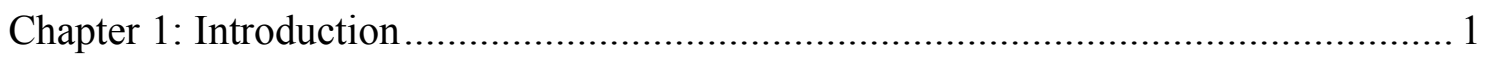

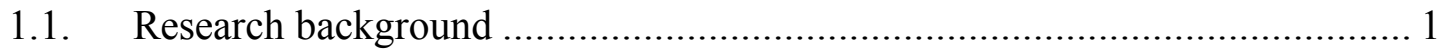

1.2. Research objectives and questions .................................................... 2

1.3. Research approach and methodology .................................................... 3

1.4. Research significance and links to development theory and practice.............. 3

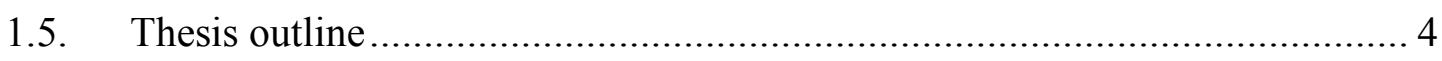

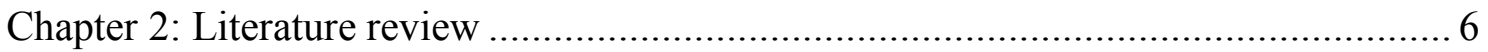

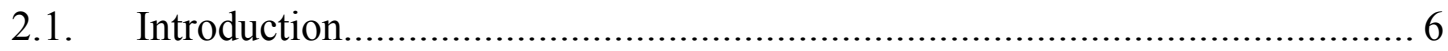

2.2. Gender and Development (GAD) theoretical approach .............................. 6

2.3. The concept of empowerment................................................................. 7

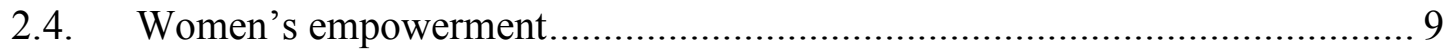

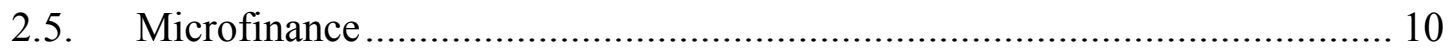

2.6. Microfinance, women's empowerment, and development......................... 12

2.7. Women's personal empowerment theoretical framework........................... 18

2.7.1. Women's personal empowerment ..................................................... 19

2.7.2. Women's personal empowerment as an analytical framework.............. 20

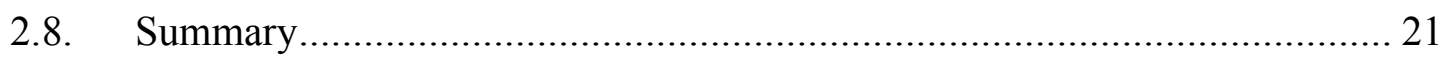

Chapter 3: Research methodology..................................................................... 23

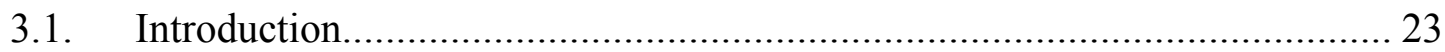

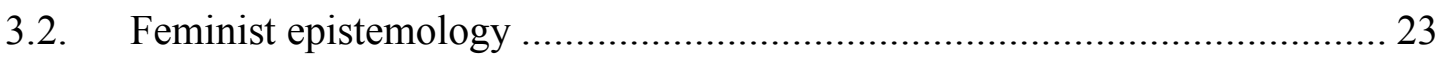

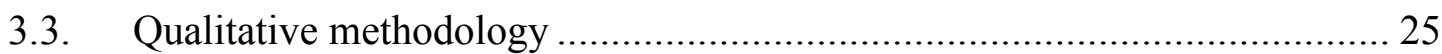

3.4. Location, recruitment of participants, and research timeline ....................... 26

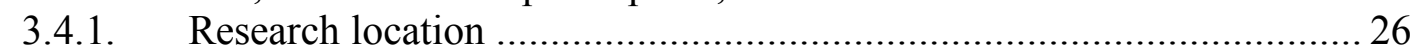

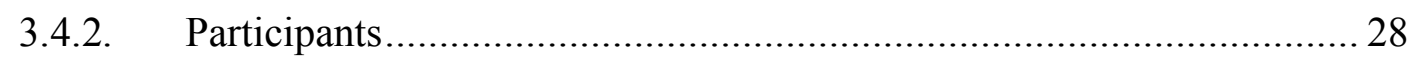

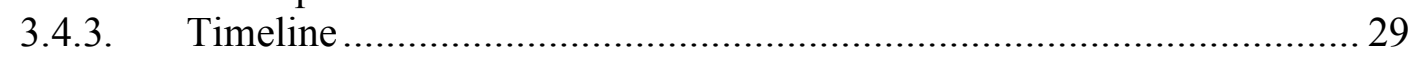




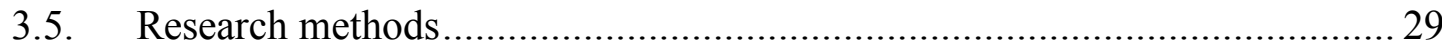

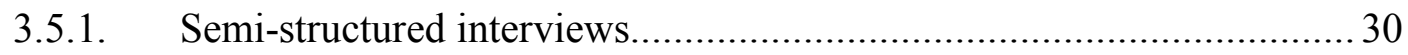

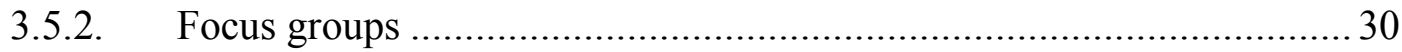

3.6. Data preparation, analysis, and interpretation......................................... 32

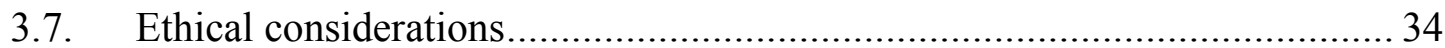

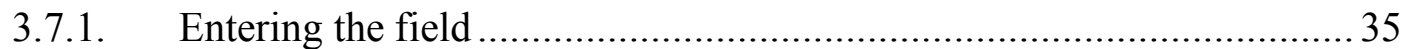

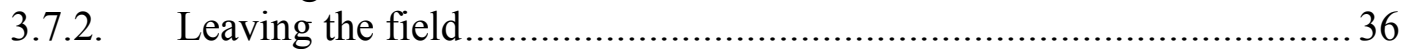

3.7.3. Interpreting the meanings and use of language .............................. 37

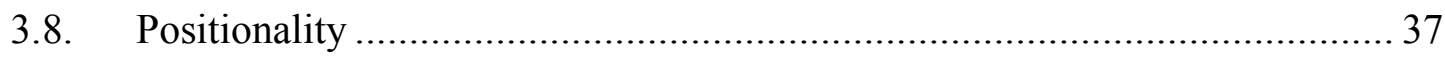

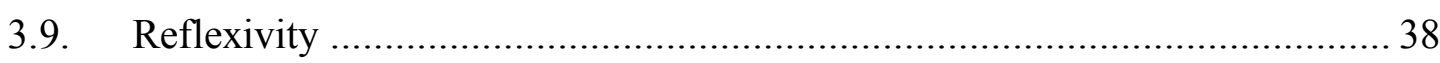

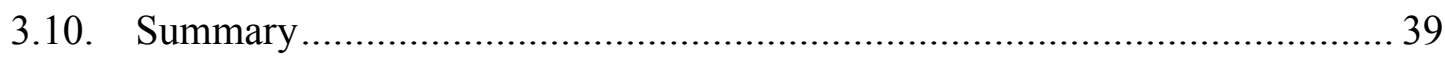

Chapter 4: The social, economic, and political contexts of Laos ............................ 40

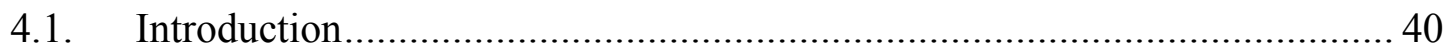

4.2. Geography, people and culture .......................................................... 40

4.3. Political system and socioeconomic development .................................... 46

4.3.1. Political system.......................................................................... 46

4.3.2. Economic reform and its effects on women.................................... 47

4.3.3. Economic growth and gender equality ........................................ 49

4.4. National policies on gender equality and international cooperation.............. 53

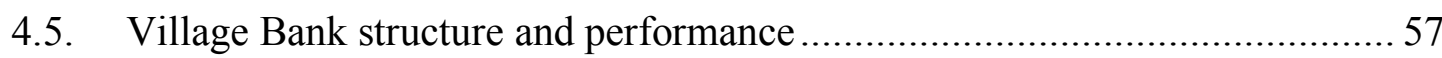

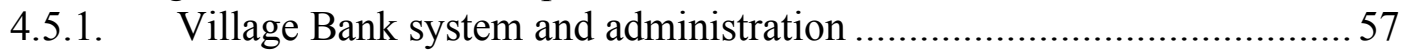

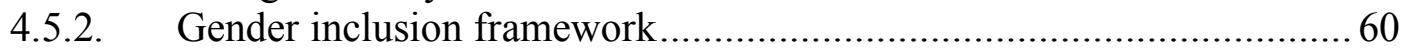

4.5.3. Village Bank performance on women's empowerment .......................6 61

4.6. Three Village Banks in Luang Namtha Province — case studies .................. 63

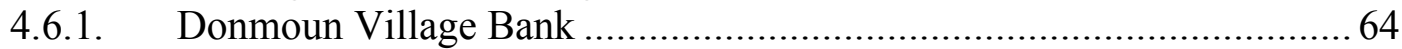

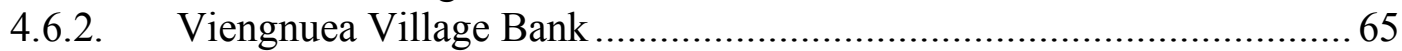

4.6.3. Namdaeng Tai Village Bank ............................................................ 66

4.7. Feminist perspectives on Laos' initiatives on women's empowerment......... 69

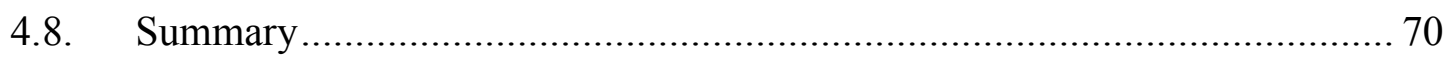

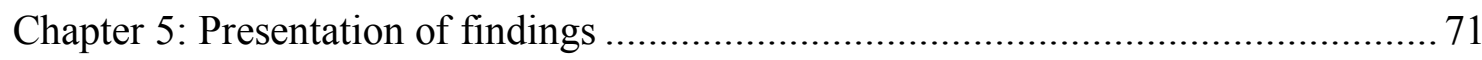

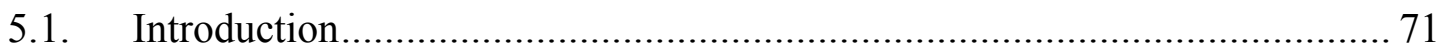

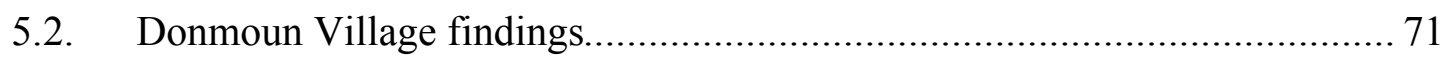

5.2.1. Khmu women's perceptions of empowerment................................. 71

5.2.2. VB benefits in respect of women's family obligations....................... 72

5.2.3. Benefits for VBC members versus ordinary VB members................... 73

5.2.4. The effect of the VB on women's mobility ....................................... 73

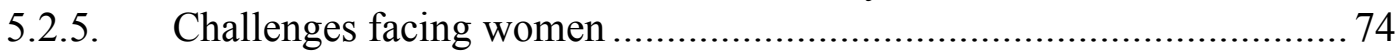

5.2.6. Women's strategies to overcome these challenges............................. 75

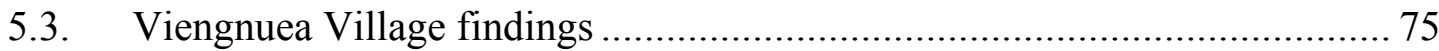

5.3.1. Tai-Yuan women's perceptions of empowerment ............................. 75

5.3.2. The effect of the VB on villagers' security ....................................... 76

5.3.3. The effect of the VB on VBC members............................................ 77

5.3.4. The limited effect of the VB on ordinary VB members ...................... 78 
5.3.5. Spiritual practice, technologies, and gender equality ......................... 79

5.3.6. Challenges facing Tai-Yuan women ................................................ 80

5.3.7. Tai-Yuan women's strategies for overcoming the challenges .............. 81

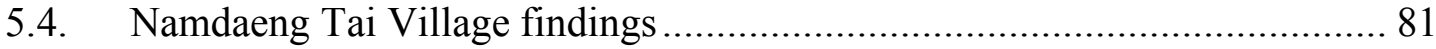

5.4.1. Hmong women's perceptions of empowerment.............................. 81

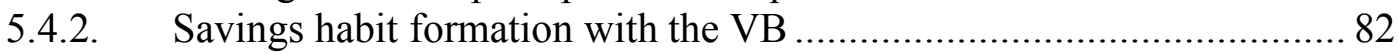

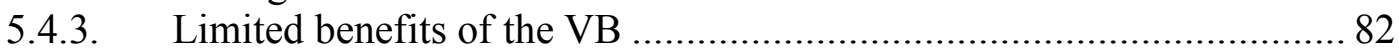

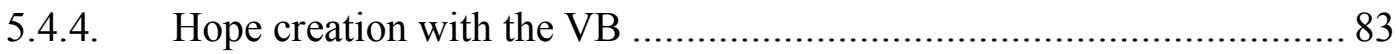

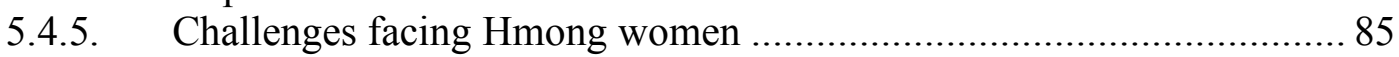

5.4.6. Hmong women's strategies for overcoming the challenges ................. 90

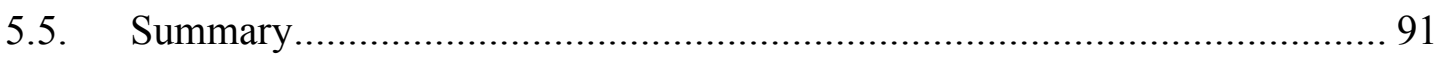

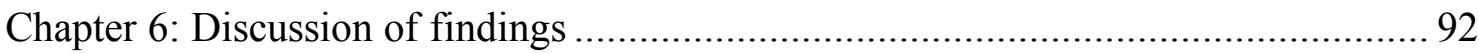

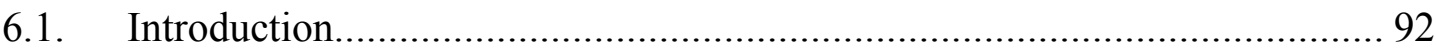

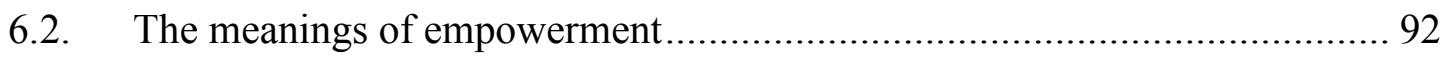

6.3. Practical and strategic gender needs................................................. 95

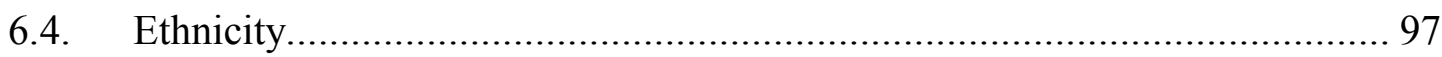

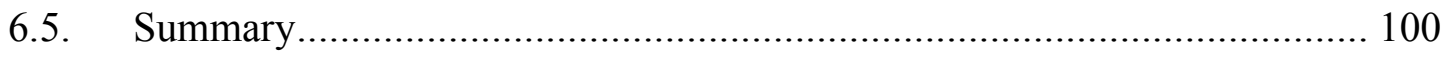

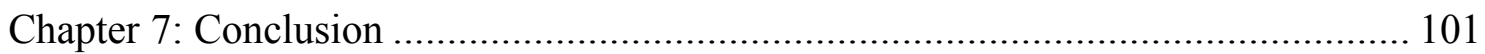

7.1. Contribution of microfinance to the empowerment of women of different

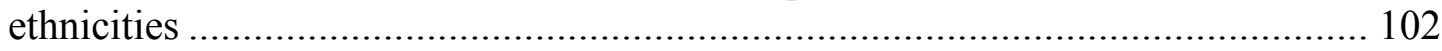

7.2. Areas for further expansion of microfinance .......................................... 103

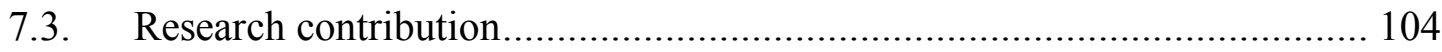

7.4. Research limitation and areas for further study .................................... 105

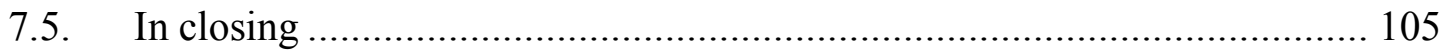

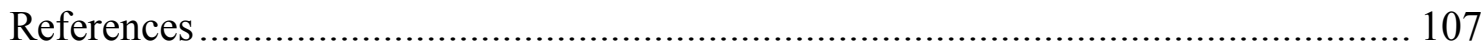

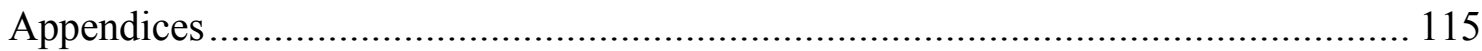




\section{List of Boxes}

Box 2.1. Some definitions of empowerment .............................................................. 8

Box 2.2. Some definitions of women's empowerment............................................... 9 


\section{List of Figures}

Figure 2.1. Women's personal empowerment analytical framework......................... 21

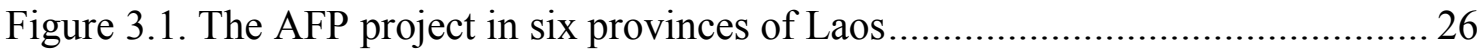

Figure 3.2. Population of Luang Namtha province by ethnicity …........................... 27

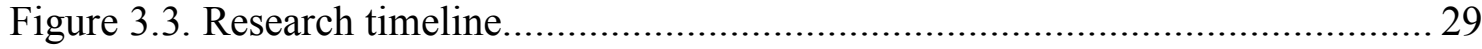

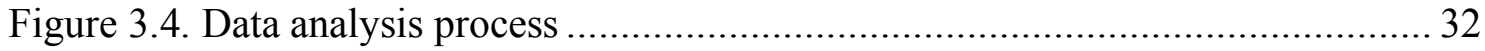

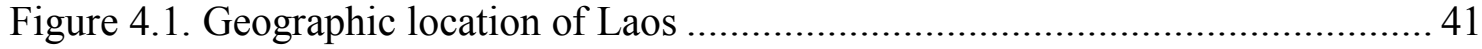

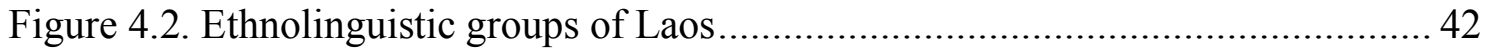

Figure 4.3. Economic structure between 2010 and 2015 ........................................ 50

Figure 4.4. The trends of employment in the three sectors from 2010 to 2015 ............ 51

Figure 4.5. Percentage of females in government organisations.............................. 52

Figure 4.6. The system of the AFP project ..................................................... 58

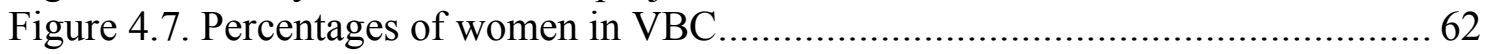

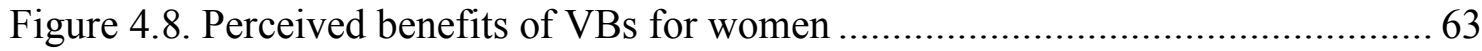

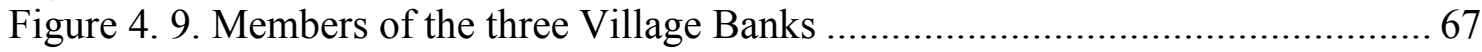

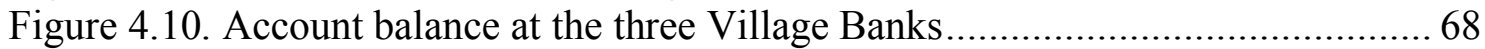

Figure 4.11. Types of loans taken by VB members .................................................. 69 


\section{List of Tables}

Table 2.1. Personal empowerment core values .................................................... 19

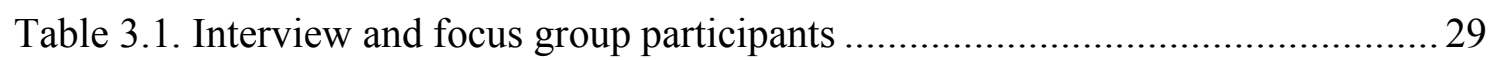

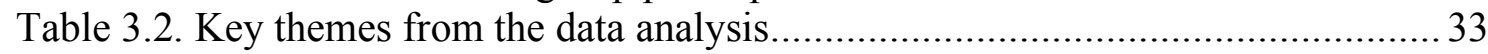

Table 4.1. Ethic groups of four ethnolinguistic families ....................................... 43

Table 4.2. Gender equality and advancement of women indicators...............................53

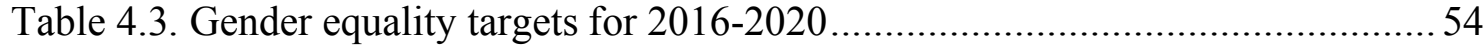

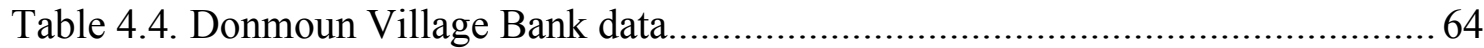

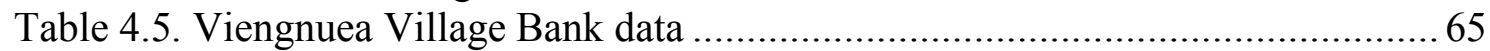

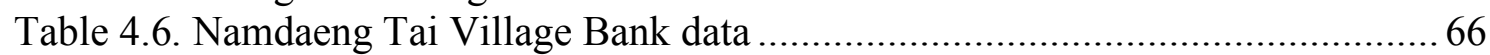




\section{Chapter 1: Introduction}

\subsection{Research background}

Since the 1980s, women's empowerment has been included in the international development agenda, and increasingly recognised for its contribution to the social and economic development necessary for poverty reduction (Malhotra \& Schuler, 2005). It is believed that the empowerment of women brings about all sorts of positive changes in society, including the closure of the gap between the rich and the poor as well as between men and women, and the promotion of sustainable, inclusive and equitable development (Nanji, 2014). These views have resulted in the ubiquitous adoption of empowerment among development interventions in order to raise public awareness and promote public involvement in policy making (Connel, 1999; Rowlands, 1999).

Despite these initiatives and efforts, the empowerment of women remains a challenge globally. Women constitute 70 percent of the world's poor (UNDP, 1995, p. 4), and form the world poorest (Noreen, 2011). Gender inequality remains common across the world, particularly in poor countries with the widest gender gaps which will likely take hundreds of years to close. The Human Development Index (HDI) rating for women remains lower than that for men across regions (UNDP, 2018). Women face significant challenges in empowerment through life. These challenges are diverse, ranging from cultural norms, social structures, and political ideologies to the orientation of development agencies (Doane \& Doneys, 2015; Geleta, 2016; Khadka, 2014; Mason \& Smith, 2003; Smith, 1989; UNDP, 2018).

After its emergence in the eighteenth century, microfinance has continued to grow and become a common tool in enabling the poor to have access to the finance, skills and knowledge necessary for poverty reduction. The industry has operated in both public and private sectors, as well as across many NGOs. It has penetrated to many of the poor and to the poorest segments of society, particularly women in developing countries (Meyer \& Nagarajan, 2006). In Laos, microfinance emerged in the 1990s, following the country's economic liberalisation in the late 1980s (MFA, n.d.). The industry continues to grow and diversify its forms and services in contributing to poverty reduction.

There have been studies on microfinance and its effect in the country. The studies tend to focus on the contribution of microfinance to poverty reduction and economic empowerment of women (Phonesavanh, 2015; Prochaska, Hauger, \& Fischer, 2012; 
Sayvaya, 2012; Sengsourivong \& Mieno, 2014). However, there is no guarantee that women will be empowered just by reducing gender disparities and increasing women's economic gains (Kabeer, 2015; Lucy, Ghosh, \& Kujawa, 2008; Oxaal \& Baden, 1997). Women's empowerment goes beyond the gender inequality commonly known as vertical disparity. It needs to be explored from a horizontal perspective focusing on age, ethnicity, class, and race (Kabeer, 2015).

Laos is faced with multiple development challenges. The country has experienced a significant advancement of women's empowerment at the central level but very little at the local or grassroots levels (ADB \& World Bank, 2012). The country's multiethnicity poses challenges for the government, development agencies, microfinance institutions, as well as the women themselves in achieving empowerment. Culture is one component that plays a significant role in gender relations (Baden \& Milward, 1995; Buvinic, 1983; Khadka, 2014; Loutfi, 1987; Noreen, 2011; Rogers, 2005). Cultural norms and values shape a common and normative belief in the way families are organised, wealth is allocated, and how the relations between men and women are defined (Smith, 1989). People make decisions based on their beliefs and on norms which have existed in their societies for many centuries (Mason \& Smith, 2003).

With the focus of current studies on microfinance and poverty (Prochaska, et al., 2012; Sayvaya, 2012; Sengsourivong \& Mieno, 2014), there has been no specific study conducted on the effect of cultural norms and values on women's use of microfinance for their own empowerment. This is noted by Doane and Doneys (2015) who state that empowerment in Laos needs to be assessed on invisible factors. Therefore, this study aims to investigate the interactions between culture, microfinance, and women's personal empowerment. This study examines three ethnic groups of women in Luang Namtha province of Laos participating in the Access to Finance for the Poor (AFP) project which supports village banks. It explores the cultural norms and values that drive women's perceptions of empowerment and use of the financial services, and explores other influential factors driving women's utilisation of microfinance for personal empowerment. Luang Namtha province was chosen as the research location given its richness in the ethnic groups involved in the project.

\subsection{Research objectives and questions}

The central aim of this research is to explore the contribution of microfinance to women's personal empowerment. It looks at the "power from within" women by investigating 
different women's meanings of empowerment, the extent to which microfinance contributes to the women's achievement of power, and any influential factors (particularly cultural norms and values) that drive the empowerment matrix.

To respond to this aim, a central research question was raised: "How has microfinance contributed to the personal empowerment of ethnic women in Luang Namtha, Laos?". To answer this key question, four sub-research questions were developed as follows:

1. What does empowerment mean for different ethnic women in Laos?

2. How has microfinance impacted their lives?

3. What are the enablers that help microfinance contribute to women's empowerment in Laos?

4. What are the challenges facing the women in using microfinance as a means for achieving empowerment? What are their strategies to overcome these challenges?

\subsection{Research approach and methodology}

As this research is to explore the perceptions and personal experiences of women in terms of empowerment, a feminist approach is employed to investigate the contribution of microfinance to women's empowerment. This study takes a qualitative approach by using semi-structured interviews and focus groups given that having these two data collection methods can provide rich data. Details of the research approach and methodology is provided in chapter 3 of this thesis.

\subsection{Research significance and links to development theory and practice}

This research links to development theory, and debates around microfinance, the empowerment of marginalised women, and development. It helps reveal the multilayered and complicated nature of empowerment (Drolet, 2005; Mayoux, 1998; Rowlands, 1997), and suggests an improved approach toward reducing women's poverty in rural Laos. As qualitative research, it provides in-depth insights into women's realities; thus adding to the discussion about the concepts of empowerment, social context, as well as ethnicity or culture within the country and other regions around the world (Creswell, 2014; Mason \& Smith, 2003). This research also provides recommendations for a national gender approach, as well as for programmes designed to effectively meet the needs of marginalised women in order to help them empower themselves. 


\subsection{Thesis outline}

This research consists of seven chapters. Chapter 2 reviews the literature relevant to microfinance and women's empowerment. The chapter begins with reviewing the Gender and Development (GAD) approach which is a foundation for women's empowerment. Then it explores the concept of empowerment, women's empowerment, and microfinance. The substantial part of the chapter is focused on reviewing the contribution of microfinance to women's empowerment. It ends with an argument for the need to study the power from within women.

Chapter 3 provides some information about the philosophy, methodology, and reflections from the fieldwork of this study. It explains the need to use feminism as the epistemology of this study. Then it explores qualitative methodology by focusing on interviews and focus groups as the two data collection methods of this study. Information on the research location, participant recruitment, data analysis and interpretation methods are also provided. The chapter ends with some reflections on the fieldwork.

Chapter 4 explores the national context, and the particular project in which the three case studies of this research were chosen from. It provides a general profile of the country and some specific insights regarding the three ethnic groups targeted in this study. It further draws the picture of Laos by exploring political and economic dimensions which have an influence on women. The national approach toward gender equality is also explored in this chapter. The chapter also explores the organisation, operation, and performance of women's empowerment in the project. It ends with some specific information on the three Village Banks chosen as case studies in this research.

Chapter 5 presents the findings of this research. It presents the findings using a thematic structure which is also based on ethnicity. The themes are organised based on the sequence of the sub-research questions. Findings with respect to the Village Banks are presented in a chronological order based on their establishment.

Chapter 6 discusses some important themes of the findings. It links the findings to extant literature in order to make arguments and produce potential answers to the research questions.

Chapter 7 answers the research questions directly, provides suggestions on areas for further development of microfinance, and explains the research contribution to theory and practice. It notes the limitations of the study, and raises some areas that need further 
investigation. The chapter ends with a personal message which derives from the experience of the research. 


\section{Chapter 2: Literature review}

\subsection{Introduction}

This chapter explores the fundamental theoretical backgrounds of women's empowerment, microfinance, and development. These reviews are fundamental for the analysis and discussion in the subsequent chapters. The chapter begins with an exploration of the theoretical standpoint of women's empowerment. It explores the concept of empowerment and women's empowerment. Then it introduces microfinance as a commonly used means for empowering women. The substantial part of this chapter examines the effect of microfinance on the empowerment of women, and thus on development. The chapter ends with an argument for the use of a women's personal empowerment approach. This approach is then explained as an analytical framework.

\subsection{Gender and Development (GAD) theoretical approach}

This subsection will explore the theoretical standpoint of women's empowerment. Rowlands (1997) noted that the "empowerment approach" emerged from a GAD (Gender and Development) perspective, and perhaps in combination with bottom-up or "actororiented" perspectives. The GAD approach emerged in the late 1970s and 1980s as a new analytical framework considering the dynamics of gender relations (Rowlands, 1997). The GAD approach emerged in response to the failure and limitations of Women in Development (WID) and Women and Development (WAD) approaches (Visvanathan, Dugan, Nisonoff, \& Wiegersma, 1997). The GAD approach argues that gender relations are at the core of social processes and social organisation and, by extension, of development. Thus, development is a complex process of individual and societal advancement in social, economic, political and cultural respects (Young, 1993, as cited in Rowlands, 1997, p. 26). The approach explains the sexual division of labour by looking at value systems. The analysis of gender relations, which are believed to be negotiated and renegotiated along the process, can lead to the understanding of relevant features such as households, the composition of the labour force, and the informal economy (Rowlands, 1997). The GAD approach suggests that gender inequalities exist in every aspect of women's lives so gender analysis must consider the complexity of people's conditions and environment (Rowlands, 1997).

According to Reeves and Baden (2000), women's empowerment in the GAD paradigm is a process towards meeting women's practical and strategic needs by addressing power 
relations. Therefore, the aim of the GAD approach is to promote gender equality in terms of opportunities and life chances (Drolet, 2005). Men and women have equal rights to make decisions for themselves and for society according to their interest (Drolet, 2005).

It can be seen that women's empowerment from this perspective is more about addressing gender relations as well as the power dynamics between men and women. Moser (1989) equates the "empowerment approach" with gender planning. This study of women's empowerment is informed by this point of view: that there are existing sociocultural structures directing the interactions between men and women, so therefore the empowerment of women can be understood and enabled through the examination of sociocultural structures.

\subsection{The concept of empowerment}

There are variations in the way empowerment is understood and defined. Empowerment can only be defined precisely depending on specific people and contexts (Reeves \& Baden, 2000; Zimmerman, 1990). Any attempt to create a universal definition of empowerment only leads to contradict the very concept of empowerment (Zimmerman, 1990). Therefore, empowerment can be defined differently. Page and Czuba (1999) suggest that empowerment is basically understood as multidimensional, social, and a process. The dimensionality is because empowerment happens within sociological, psychological, economic, and other dimensions. Empowerment is also believed to happen at many levels, including individual, group, and community levels. In addition, empowerment is a social process which can be improved in relationships with others, and develops as it is worked through within specific contexts and with specific people. It is also important to note that there is a connection between the individual and community (Page \& Czuba, 1999). 
- Empowerment is "the ideal combination of change, choice and power. It is a process of change by which disempowered gain control and ability to make decisions that affect their life" (Cheston \& Kuhn, 2002, p. 12).

○ Empowerment is “the process of enhancing an individual's or group's capacity to make purposive choices and to transform those choices into desired actions and outcomes" (Alsop, Bertelsen, \& Holland, 2006, p. 1).

○ Empowerment is "a process by which individuals gain mastery and control over their lives and a critical understanding of their environment" (Rappaport, 1987, p. 122).

○ Empowerment is "the process by which an individual participates in community and gains control and resource mobilisation skill as an outcome" (Perkins \& Zimmerman, 1995, p. 570).

From these definitions of empowerment (in Box 2.1), it is obvious that empowerment is a process that disempowered people take to achieve the capacity to take control over their own lives. However, according to Rappaport (1987), it is crucial for the disempowered to thoroughly be aware of their own environment, and the conditions that keep controlling them. This is consistent with what Freire (1970) argues: that individuals can be empowered when they understand the conditions that keep oppressing them. In his study, Henry (2011, p. 251) employed empowerment principles from key community psychologists who defined empowerment as "the ability of individuals to change the conditions that oppress them by having a psychological sense of personal control and a concern for having a social influence, political power, and legal rights". Therefore, it is argued that any merely resource-providing interventions cannot succeed unless selfconfidence in using the resources is raised, and unless self-realisation is achieved (Presser $\&$ Sen, 2000).

More precisely, some sociologists contend that empowerment is a process towards achieving four aspects of power: "power within", "power to", "power with", and "power over" (Mayoux, 1998, p. 45; Rowlands, 1997, p. 13). These aspects of power were initially introduced by Williams, Seed, and Mwau (1994, as cited in Oxaal \& Baden, 1997, p. 1) who defined "power over" as the ability to make a change against nomination or subordination; "power to" as the ability to make decisions, solve problems, and be creative and enabling; "power with" as the ability to have social networks and participate 
towards common collective goals; and "power within" as self-confidence, self-awareness and assertiveness. The "power from within" includes the ability to recognise and be aware of the conditions, environment, and power relations around oneself, then being confident to take actions to change them (Mayoux, 1998; Rowlands, 1997).

\subsection{Women's empowerment}

From the above section, it can be seen that empowerment can be defined differently according to specific people and contexts. Empowerment also means a process of achieving different aspects of power. This subsection will explore women's empowerment specifically.

\section{Box 2.2. Some definitions of women's empowerment}

○ Women's empowerment is "a process whereby women become able to organise themselves in order to increase their own self-reliance, to assert their independent right to make choices and to control resources which will help them to challenge and eliminate their own subordination" (Keller \& Mbwewe, 1991, as cited in Rowlands, 1999, p. 146).

- Women's empowerment is "the process through which women gained the capacity for exercising strategic forms of agency in relation to their own lives as well as in relation to the larger structures of constraint that positioned them as subordinate to men" (Kabeer, 2012, p. 7).

○ Women's empowerment is "women's sense of self-worth; their right to have and determine choices; their right to have access to opportunities and resources; their right to have the power to control their own lives, both within and outside the home; and their ability to influence the direction of social change to create a more just social and economic order, nationally and internationally" (United Nations, 2001).

○ "Women's empowerment should lead to the liberation of men from false value systems and ideologies of oppression. It should lead to a situation where each one can become a whole being regardless of gender, and use their fullest potential to construct a more humane society for all” (Batliwala, 1994, p. 131). 
Rowlands (1997, p. 13) and Mayoux (1998, p. 45) perceived women's empowerment as a process toward achieving four aspects of power, known as "power within", "power to", "power with", and "power over"; whereas Kabeer (2005) conceptualised the empowerment of women as the interrelations between agency, resources, and achievements. Despite some variations in how empowerment is conceptualised, the three authors believed that a "sense of agency" is the core of the empowerment process, and this can be triggered by the achievement of "power from within". This aspect of power is achieved when women become aware of themselves and their surrounding environment. With this power, women start to see themselves as just as important and valuable, and as deserving of equal respect and opportunities as others. Rowlands (1997) believes that this fundamental aspect of power can encourage women to work towards other aspects of power, including taking collective action under the "power with" to create changes in society. This can be considered as women's achievement of political power that could eventually lead to "power over" where women are able to act without domination and oppression, or even against resistance (Rowlands, 1997).

\subsection{Microfinance}

This subsection will introduce microfinance as a well-known intervention in poverty reduction including women's empowerment. It is well known as a means for helping empower women despite various arguments against it. Before being recognised widely today, the concept of microfinance has a long history which can be traced back to the eighteenth century (Meyer \& Nagarajan, 2006). There were initially financial interestfree schemes for the poor in Europe in the 1700s and 1800s. Gradually, these charitybased arrangements started to evolve, widen, and modify. In Germany, savings and credit cooperatives for rural and urban people were created with the purpose of enabling selfreliance by connecting households with banks to ensure outreach and savings mobilisation among local people. These cooperatives later modified themselves to become more than a thousand banks by 1920 (Robinson, 2001).

Microfinance from the twentieth century is identified as a modern model of microfinance. The birth of modern microfinance is generally attributed to the creation of the Grameen Bank by the economics professor Muhammad Yunus in 1983 in Bangladesh. However, Robinson (2001) argues that modern microfinance was first found in Indonesia where a private bank, Bank Dagong Bali (BDB), was identified as the oldest formal microfinance institution providing savings and loans without relying on any subsidy since 1970. It is 
seen from the study of Meyer and Nagarajan (2006) that modern microfinance has experienced a rapid growth in the twentieth century, particularly in the developing world. There were modes of microfinance emerging in Latin America such as group loans for poor women in microbusiness, village banks with smaller average loan sizes, and other microfinance operations. With the continuous emergence and development, the number of microfinance institutions reached more than 1,000 before 1992 with over 1,000 members per institution (World Bank, 1996).

Therefore, according to Geleta (2016, p. 7), microfinance is defined as "a small-scale financial service that includes lending to and saving from low-income clients who are generally regarded as poor. Some microfinance institutions also provide insurance and payment services". The definition of microfinance has gone through an evolution. Initally it referred to microcredit that non-government organisations or specialised financial institutions lent to poor people and small businesses with some form of collateral (Meyer \& Nagarajan, 2006). Today, the concept includes a much broader range of services like loans, savings, insurance, remittance transfers, and even pensions available at many types of financial institutions and organisations (Meyer \& Nagarajan, 2006).

It is also argued that microfinance has been shaped by three paradigms (Drolet, 2010; Mayoux, 2002). These paradigms include financial self-sustainability, poverty alleviation, and feminist empowerment. Mayoux (2002) contends that microfinance in the financial self-sustainability paradigm operates under a neoliberal perspective in which a huge number of poor people are targeted under terms which lead to the microfinance institution making a profit. Thus, microfinance in this paradigm aims to increase the number of its programmes, and becomes linked to the private banking sector. These private microfinance programmes are funded through international financial markets, rather than by donors and development agencies. This informs the financial delivery policies of the microfinance industry in terms of cost effectiveness. Poor women, in this paradigm, are targeted as new unexplored clients. Therefore, women's empowerment in this paradigm is perceived as "the expansion of individual choice and capacities for selfreliance within existing structures, rather than examining underlying constraints" (Drolet, 2010, p. 218). This paradigm assumes that women's access to finance can reduce their poverty, and enable them to empower themselves. Microfinance in this paradigm aims to address women's practical needs rather than strategic needs (Drolet, 2010). 
Secondly, the poverty alleviation paradigm seems to provide a better version of microfinance compared to the financial self-sustainability paradigm. Microfinance programmes in the poverty alleviation paradigm are subsidised by donors, and provide training and social services to their members with a view of reducing household vulnerability and poverty, and targeting women (Mayoux, 2002). Microfinance in this paradigm believes that once women's practical needs are addressed, it will encourage women to make their way through overcoming gendered issues to achieve empowerment. This paradigm assumes that women's and household's interests are similar so that everyone in the family can benefit from the credit equally. Microfinance in this paradigm, therefore, is focused on household poverty reduction rather than women's empowerment (Mayoux, 2002).

Finally, the feminist empowerment paradigm recognises the complexity of women's empowerment (Mayoux, 2002). Microfinance in this paradigm operates based on equity, empowerment, and equality. Women's empowerment is understood from the GAD perspective in which empowerment goes beyond the economic achievement of women. It is more about power relations in society as a collective rather than women or men as individuals (Mayoux, 2002). Therefore, microfinance in this paradigm aims to support women's strategic interests by evaluating its contributions to women's development rather than economic gains (Tesoriero, 2006).

\subsection{Microfinance, women's empowerment, and development}

Earlier subsections have examined the concept of women's empowerment which was developed under the GAD theoretical approach, and microfinance as a means for women's empowerment. This subsection will review the extent to which microfinance helps empower women, and by extension contributes to development. The contributions of microfinance to women's empowerment and development have been argued from various points of view. Those who argue that development is to serve people, see microfinance as being an opportunity for people to be included in the heart of development processes and policies formation. Those who care for women's rights see microfinance as being able to empower women by focusing on gender issues and development. Those who think poverty reduction is important see microfinance as a solution for improving livelihoods of the poor, particularly during economic hard times. In addition, microfinance is believed to benefit the most disadvantaged regions and to contribute to long-term development (Nader, 2008). 
However, a body of research suggests that women can only be empowered if they become aware of themselves and their environment (Henry, 2011; Rappaport, 1987; Rowlands, 1999). The intervention of microfinance has helped women gain a sense of "power within", which is a power allowing women to identify their capacity and capability within themselves in order to take actions to achieve their goals (Kabeer, 1998). This is the power that leads women to gain another power known as "power to", which allows women to act against existing social structures, and to influence those structures for change (Rowlands, 1999, pp. 143-44). A study conducted in northern Tanzania about the effect of two grassroots NGOs on women's empowerment also confirms that an increase in personal agency can lead to changes at societal level, including political and gender norm changes. A raft of changes can result in social transformation (Goldman \& Little, 2015). Therefore, internal change can be seen as a prerequisite for women to actualise empowerment.

In addition, several studies argue that women's internal achievement is enabled through group-based microfinance. The group-based approach is seen to contribute to psychological empowerment of women (Hansen, 2015). Qualitative analysis in a systematic review of the effectiveness of women's economic Self-Help Group (SHG) programmes in many developing countries shows that SHG programmes have contributed to psychological empowerment of women (Brody, et al., 2015). Another study conducted in Sri Lanka confirms that treatment group women experienced greater psychological empowerment compared to those of the control group. This was thought to be because women's involvement in microfinance programmes exposes them to training and grouping in which the social network provides them with a sense of belonging, identity, and protection (Hansen, 2015). Therefore, group-based microfinance is fundamental for women to develop political empowerment (Brody, et al., 2015).

Despite these findings, other studies argue that group-based microfinance does not always work as expected. There are cases where credit-based groups dissolved after their formation because group members did not feel comfortable coming to and working in the group. Many of them turned to friends, relatives, and money lenders for credit, despite embarrassment and high interest (Banerjee, Duflo, Glennerster, \& Kinnan, 2015). Another study shows that the group-based approach can result in even more male violence against women, given that the approach is to create group supervision and discipline in order to ensure repayment of loans. Without explicit intervention strategies, group-based 
microfinance is just a new exploitative and disempowering structure that continues to exploit and marginalise women (Geleta, 2016).

Similarly, Ahmed, Chowdhury, and Bhuiya (2001) contend that women are suffering emotional stress in microfinance programmes while women's emotional well-being tends to receive no attention. Their study of women participating in a microfinance programme for two years in Matlab of Bangladesh revealed that the programme female members were suffering more emotional stress which was basically linked to their programme membership. In contrast, non-programme members suffered less emotional stress, and the emotional stress they did suffer was linked to other factors such as marital issues. The authors also argue that women usually have high aspirations, so their limited achievements cause anxieties, tensions, domestic violence, and emotional and physical suffering.

Beside these effects, other studies found some effects of microfinance on women's capacity and capability. The findings of a study in Nepal show that the effect of microfinance on aspects of women's empowerment varies (Khadka, 2014; Swain \& Wallentin, 2009). The microfinance programme members were found to have obtained communication and decision-making abilities, given that the women had become confident and gained recognition among family members (Khadka, 2014). Women were found to be able to make decisions either unilaterally or bilaterally with their counterpart family members (Lucy, et al., 2008). A quantitative synthesis of data from a study of SelfHelp Groups (SHGs) in several developing countries contends that the variations in the impacts of SHGs on empowerment are due to programme design and social context. A qualitative synthesis of data from the same study acknowledges benefits to women's financial and decision-making abilities. It also acknowledges improved connections with, and respect and support from, families and community members. It suggests this enables women's economic, social, and political empowerment (Brody, et al., 2015).

Similarly, microfinance has been found to contribute to an increase in women's ownership of property, and by extension to economic empowerment. A Bangladeshi NGO working on women's gender awareness and capacity building was found to have enabled benefits in terms of raising household income and enabling women's land ownership through use of microfinance. Most of the women participating in the programme dominated decision-making in the family. The women took control of the spending of the income from their own businesses, of the loan for investment, and of 
general spending of household income (Lucy, et al., 2008). In addition, there have been studies confirming that women's empowerment can be triggered by women's capability in economic aspects. A study on the successes of the Grameen Bank and the Bangladesh Rural Advancement Committee (BRAC) in empowering women in Bangladesh shows that the achievements of these two organisations in helping women in rural Bangladesh achieve empowerment are attributed to the provision of economic opportunities, and the managerial skills of the organisations (Hashemi, Schuler, \& Riley, 1996). It is not suggested that these programmes addressed sociocultural norms. It has been argued that lack of economic opportunities is a key barrier for women's empowerment. By getting involved in the programmes, aspects of women's empowerment such as mobility, purchase capacity, household decision-making, control over productive assets, and legal and political participation increase. The programmes can also help women achieve transformation given that having access to crucial economic resources can lead to a change in gendered power relations. This in turn enables women to have more decisionmaking power and status in families, so that even in a patriarchal society men will accept women as resource controllers. However, the study suggests that any effort to deal with a patriarchal system should be taken by other NGOs or state agencies rather than microfinance programmes (Hashemi, Schuler, \& Riley, 1996).

Economic achievements can fulfil women's practical needs rather than their strategic needs. Moser (1993) explains:

Practical gender needs are the needs women identify in their socially accepted roles in society. Practical gender needs do not challenge the gender divisions of labour or women's subordinate position in society, although rising out of them. Practical gender needs are a response to immediate perceived necessity, identified within a specific context. They are practical in nature and often are concerned with inadequacies in living conditions such as inadequate water provision, health care, and employment (p. 39).

Strategic gender needs are the needs women identify because of their subordinate position to men in their society. Strategic gender needs vary according to particular contexts. They relate to gender divisions of labour, power, and control, and may include such issues as legal rights, domestic violence, equal wages, and women's control over their bodies. Meeting strategic gender needs helps women to achieve greater equality. It also changes existing roles and therefore challenges women's subordinate position (p. 40). 
A study conducted in Mumbai in India on the effectiveness of a "Credit-Plus" model, in which social and financial services were integrated in order to better serve people, particularly women, revealed that there was no evidence of political actions among the women to challenge social injustice and inequality (Krenz, Gilbert, \& Mandayam, 2014). This was the case despite the model's help in addressing household vulnerability, the inability of women and families to meet their basic needs, as well as in improving relationships in the household and the community. In addition, women's increasingly important role in financial earning, and better interpersonal relationships in the family, appeared to create no change in the labour division between men and women (Krenz, Gilbert, \& Mandayam, 2014). There are studies confirming that microfinance is not capable of empowering women, despite some positive signs of women's increasing role in their children's education, and increasing financial contribution to households and national growth (Nader, 2008; Panjaitan-Drioadisuryo \& Cloud, 1999). Microfinance has been seen as effective in poverty reduction rather than in long-term and sustainable empowerment of women. A similar argument made in a study in rural India was that women borrowed money which contributed to household assets and incomes rather than to women's strategic needs. There was no change in women's status in the household. This is combined with the fact that women are excluded from property ownership so that they become disempowered instead (Garikipati, 2008).

Different studies blame the failures of microfinance on dissimilar factors. Nader (2008) attributes the insignificant achievement of empowerment to women's lack of necessary skills that can make the loan profitable. Another study in rural Bangladesh shows that the rural microfinance programmes tend to benefit men and non-poor people rather than women, given that the credit borrowed by men generates more income than that borrowed by women (Ocasio, 2016). This difference is believed to be caused by education as men are more educated than women. Entrepreneurial skills, which are necessary for success, are seen in men more than in women (Ocasio, 2016). In addition, a study of 26 microfinance projects across 22 African, Eastern European, Latin American, and Asian countries revealed that the institutional environment and women's performance at microfinance institutions act in opposite directions (Boehe \& Cruz, 2013). Women's better performance at microfinance institutions means that the institutional environment is weak, given that women do their best at microfinance institutions in order to keep the door of the microfinance institution open while other sources of support are not available for them. A weak institutional environment excludes women from having access to 
important resources and services such as the financial mainstream and education. In this situation, women are very likely to seek help from people around them, and take high responsibility for their debt where microfinance institutions are the only option left. This can be connected to the motive of microfinance institutions to target women for their financial sustainability purposes, rather than for the purpose of empowering them (Boehe \& Cruz, 2013). This argument is reinforced by a study conducted by Mazhar Siraj (2012) who analysed existing literature and found that microfinance fails to enable long-term and sustainable empowerment of women because of its commercialisation in which women are no longer their priority target. Another study in Indonesia confirms that women can benefit the microfinance programmes by helping reduce delivery costs and debt risks (Panjaitan-Drioadisuryo \& Cloud, 1999).

In addition, other studies found that cultural norms and values are barriers to the empowerment of women. A study on the impact of a microfinance model in Nepal found that women's loans were used to cover daily expenses of the family so there was no significant impact on the relations between men and women. The women could not take control over the loan due to the patriarchal traditions (Khadka, 2014). Even group-based microfinance is challenged by existing social norms and culture despite its various contributions to women's empowerment (Brody, et al., 2015; Swain \& Wallentin, 2009). Men's violence against women may decline during the first period of time when women still keep close and regular contact with other programme members, but it may come back or even become worse for those who no longer depend on the programme for conducting their own income-generating activities. There are also cases when women who earn a modest income suffer violence more regularly than those who earn nothing. Only those who are very successful in economic activities are much more empowered and have more control over their incomes and properties (Schuler, Hashemi, Riley, \& Akhter, 1996). A study targeting women in five Asian countries (India, Malaysia, Pakistan, the Philippines, and Thailand) found that social context has much more influence on women's empowerment than individual characteristics do (Mason \& Smith, 2003). A recent study in Ethiopia also argues that current microfinance programmes underestimate the influence of cultural norms and values on the empowerment of women. This can be seen as a key reason why current microfinance programmes fail to create a transformation among women. Women's ownership of important economic resources cannot lead to their empowerment if the ownership becomes a new form of exploitation of women (Geleta, 2016). 
However, the notion that women's empowerment can be achieved by challenging cultural norms and values has been questioned. Ahmed, et al. (2001) conducted a study on the impact of microfinance on women's emotional well-being in Bangladesh, and found that changing cultural norms from patriarchy to allowing women to be heads of families would just increase women's emotional stress. Meanwhile, the stress would be worse among unmarried women and single parents. This is because these women normally do not have the skills and capabilities necessary to manage their families due to their lack of education (Ahmed, et al., 2001).

From these findings, it can be seen that the question of whether microfinance has an empowering effect on women, and then contributes to development, is hard to answer. This is due to the multilayered and multidimensional nature of empowerment that makes exact measurements of empowerment impossible. In Linda Mayoux's (1998) acknowledgement of gender impact assessment, she explained:

Similar conditions of credit delivery, groups of formally similar structures or similar training programmes may have very different impacts depending on whether they are being implemented within a programme framework emphasising empowerment or emphasising financial sustainability.... Women's access to or control of income cannot be inferred from financial statistics on women's take-up of financial services, repayment levels or enterprise performance... Women are not a homogeneous group as assumed in many impact studies. Programme impacts typically differ between women (pp. 40-41).

She also contends that contextual and programme factors need to be studied thoroughly to understand their effects on different dimensions of empowerment.

\subsection{Women's personal empowerment theoretical framework}

In the earlier subsections, I have explored the effects, either positive or negative, of microfinance on women's empowerment. It has been seen that women's empowerment is contextual and multidimensional, and that this research is not capable of covering all aspects of empowerment. Hence this study only focuses on personal empowerment, which is also known as the "power within", in which women are believed to be initially aware of themselves in the social context, and upon this awareness, actions are taken (Kabeer, 1994, 2005; Rowlands, 1997). This subsection starts by introducing women's personal empowerment and its components. Then it will explore women's personal empowerment as an analytical framework. 


\subsubsection{Women's personal empowerment}

According to Rowlands (1997), internal psychological and psychosocial changes in women are preconditions for the whole remaining process of women's empowerment. These fundamental changes include self-confidence, self-esteem, a sense of agency, and dignity. It is argued that women's confidence and dignity alone cannot trigger the process of empowerment unless a sense of agency is achieved. Women have a sense of agency when they are aware of themselves, are able to act, and can enable changes in their social context. Rowlands (1997) argues that changes in women's self-perception can be achieved, and "internalised oppression" can be mitigated if these fundamental aspects of empowerment are promoted and increased. This can enable the increase in "power within" and "power to" enabling women to create new possibilities and changes in existing social structures. The development of these key inner aspects of empowerment is determined by encouraging and inhibiting factors: "If an empowerment approach is to be effective, it needs to foster the development of the core aspects of empowerment" (Rowlands, 1997, p. 134). The components of personal empowerment are listed in Table 2.1 as follows:

Table 2.1. Personal empowerment core values

\begin{tabular}{cl}
\hline Core values: & $\begin{array}{l}\text { Self-confidence, self-esteem, sense of agency, sense of 'self' in } \\
\text { wider context, and dignity }\end{array}$ \\
\hline Inhibiting factors: & $\begin{array}{l}\text { Fatalism, active opposition by partner, health problems, poverty, } \\
\text { dependency, lack of control over use of time, lack of control over } \\
\text { fertility/constant childbearing, childcare obligations, and male } \\
\text { control over income }\end{array}$ \\
\hline Encouraging & $\begin{array}{l}\text { Activity outside the home, being part of a group and participating } \\
\text { in its activities, ending isolation, travel, wider friendships, time } \\
\text { for 'self', sharing of problems/support, and development of } \\
\text { literacy skills }\end{array}$ \\
\hline Personal changes: & $\begin{array}{l}\text { Increased ability to formulate and express ideas and opinions; } \\
\text { increased ability to participate in and influence new spaces; } \\
\text { increased ability to learn, analyse and act; increased sense that } \\
\text { changes are possible; increased ability to organise own time; } \\
\text { increased ability to obtain/control resources; increased ability to } \\
\text { interact outside the home }\end{array}$ \\
\hline
\end{tabular}

Source: adapted from Rowlands (1997, p. 112)

\section{Explanation of the core values}

This subsection will ensure our understanding about the meaning of each core value given that a proper understanding of these values can enable correct measurements and evaluation: 
- Self-confidence: The term confidence is derived from a Latin word, fidere, which means "to trust". Thus, "self-confidence" means the trust in oneself in terms of one's abilities or aptitude to be successful. Self-confidence makes a person ready to overcome challenges, seize opportunities, and take responsibility for things (Burton, 2015).

- Self-esteem: The term esteem is derived from a Latin word, aestimare, which means "to appraise, value, rate, weigh, estimate". Thus, self-esteem is a cognitive and emotional appraisal of one's own worth (Burton, 2015).

- Sense of agency "refers to the experience of controlling one's own actions, and, through them, events in the outside world" (Haggard \& Chambon, 2012, p. R390). Thus, sense of agency is what most people feel when a particular product or event is the result of their contribution or actions (Haggard \& Chambon, 2012).

- Sense of 'self' in wider context: Sense of self is the perceptions of ourselves that are shaped by our observations of and interactions with others, rather than created in isolation. Our "social reality" is created by others; this means that others shape our thinking, feeling, and actions (Hardin \& Higgins, 1996, as cited in Jhangiani, Tarry, \& Stagnor, 2015). Our sense of self is shaped by the perceptions of others about us, by comparisons with others, by the groups we belong to, and by the audiences we have. Therefore, social situation determines our self-concept and self-esteem (Jhangiani, et al., 2015).

- Dignity: Someone's dignity being honoured means they are treated by others with respect, which usually means their uniqueness and differences are recognised. Some ways of showing respect for others include "listening, engaging others in dialogue, trusting others' wisdom, showing regard for differences, and not being judgemental about others" (Parse, 2016, p. 261).

\subsubsection{Women's personal empowerment as an analytical framework}

Women's personal empowerment can be used as an analytical framework. This framework can help me, as the researcher, explore the spiritual strength and uniqueness that make individual women who they are (Rowlands, 1997). The framework is capable of enabling the researcher to look at women's own experiences of gender subordination and how such subordination is maintained. Women's struggle to achieve power is worth studying given that "such power cannot be given; it has to be self-generated" (Kabeer, 2005; Rowlands, 1997, p. 21). Therefore, by using this framework, I can particularly 
analyse the cultural factors that either enable or hinder the process of women's personal empowerment. The framework is illustrated in Figure 2.1 below.

Figure 2.1. Women's personal empowerment analytical framework

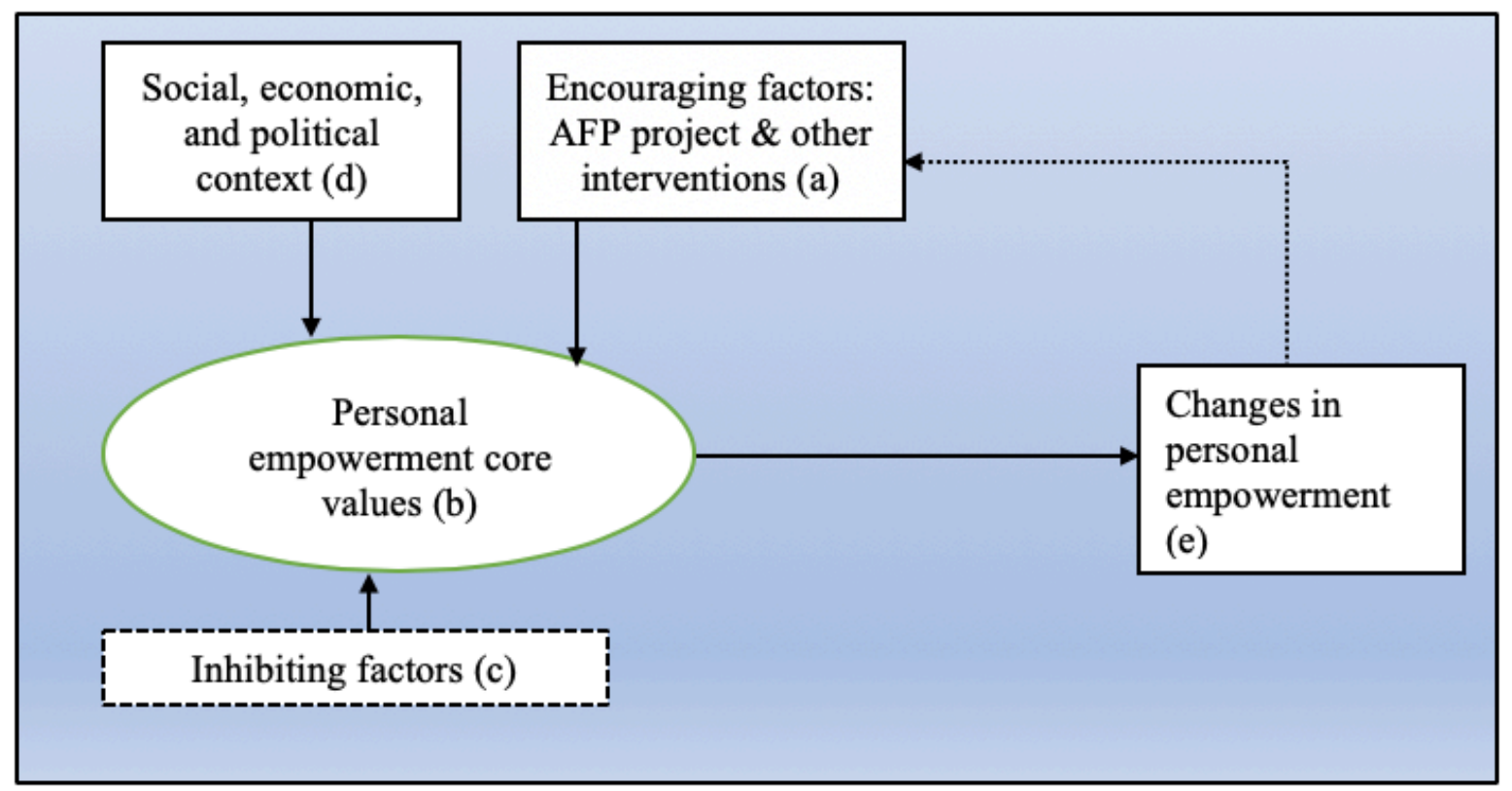

Source: adapted from Rowlands (1997, p. 112)

This research focuses on five components. These components are: (a) encouraging factors, which include the interventions of the AFP project and the Lao Women's Union; (b) personal empowerment core values; (c) inhibiting factors, particularly cultural norms and values; (d) social, economic, and political context; and (e) changes in personal empowerment. This research will look at how component (a) contributes to the achievement of component (b). Then it will examine the effects of components (c) and (d) on the effectiveness of component (a) in achieving component (b). Furthermore, the study will look at how component (b) leads to component (e). Finally, any effect of component (e) on component (a) will be discussed.

\subsection{Summary}

In this chapter, I have shown that the concept of women's empowerment emerged from the Gender and Development perspective. The examination of the concept of empowerment leads to a deeper understanding of women's empowerment. To help women achieve empowerment, microfinance has also been discussed as a popular means used by many development initiatives to address gender inequality and marginalisation. The chapter also reveals that there is no single answer with respect to the contribution of microfinance to the empowerment of women, given that empowerment is multilayered 
and multidimensional. Women's empowerment cannot be measured precisely and universally due to its contextual nature.

This chapter also suggests that women's personal empowerment needs to be studied given that it is the central part of the whole empowerment process. Thus, women's personal empowerment is considered as both the focus and conceptual framework of this study. It is used as an analytical tool due to its capability of exploring gender relations and their effects on the power from within women. This framework will inform the epistemology, methodology, and fieldwork approach in the next chapter. 


\section{Chapter 3: Research methodology}

\subsection{Introduction}

This chapter is focused on the methodology used in this research, the fieldwork, and the methods used for data analysis. It explores feminist epistemology which is used as a basis for data interpretation and knowledge construction in this study. The chapter explains the use of qualitative methodology, and data collection through interviews and focus groups. Research location, participant recruitment, and some reflections on the fieldwork are also included in this chapter. The chapter also reveals the data analysis and interpretation methods.

\subsection{Feminist epistemology}

Epistemology is a branch of philosophy, and has its roots in Greek language: "episteme and logos". The former means knowledge while the latter means explanation. Epistemology is the logic justifying a belief or justifying knowledge and the process of how the belief or knowledge is gained. It presents the question: "How do we know what we know?" (Stone, 2008). It is about how we believe something as the truth (Hull, 2015), and how legitimate knowledge of the world is acquired (O'Leary, 2007).

This study looks at women's personal empowerment from the perspective of feminist epistemology. Feminist epistemology is a social epistemology that studies the relevance between gender and knowledge given that gender is defined by social relations rather than individuals (Grasswick, 2011). More than exploring women's ways of knowing, feminist epistemology examines the connection between gendered power relations and knowledge. Feminist epistemologists believe that gendered power structures determine the production and practices of knowledge (Grasswick, 2011). Gender must be seen as part of the interactions of various components and layers rather than in isolated individuals (Alcoff \& Potter, 1993).

Grasswick (2011) contends that despite variations and even significant differences in views among feminist epistemologists, the concept "situated knowing" has been central in feminist epistemology. This means that one's social location determines and limits one's knowing (Haraway, 1988). The social location shapes knowledge production and practices which matter socially and politically. With situated knowing, the influence of masculine bias on knowledge production and practices can be articulated. Knowledge can 
be obtained from marginalised people given that they can know better within their social location (Grasswick, 2011). Even the objectivity of knowledge can be increased if research is conducted from women's lives (Harding, 1991). By exploring women's lives, researchers can assess the philosophical position of marginalised social groups. This can help researchers identify their own pre-assumptions of a particular culture in research. Harding further contends that doing research from women's lives in households can enable the impact of modernisation and technological developments on women to be identified.

Situated knowing was used as the situated communities approach by Grasswick (2011) in studying the effect of Agent Orange in Vietnam. She argues that the situated communities approach can both give researchers a better epistemic lens and a more effective methodology. According to her, purely objective knowledge is unachievable due to the lack of purely objective knowers, given that every knower gains knowledge within their own social location and through their own experiences. Grasswick explained that situated knowing is, on the one hand, epistemological because it provides researchers with the way to achieve more objective knowledge, and helps explain how individuals and groups view the world in a particular way due to their physical location. On the other hand, situated knowing is methodological because it helps produce knowledge about the world, and improve our interactions with the world.

In the situated communities approach, the research location needs to be studied given that it is as important as the situated knowledges themselves (Harding, 1991). Lorraine Code (2006) also argues that social and physical location is a place to be researched instead of being a place from which knowledge is generated. Therefore, it is seen that the concept situated knowing suggested by feminist epistemologists provides a framework for us to think about how gender and location determine epistemologies, methodologies and needs (Grasswick, 2011). Grasswick argues that research needs to be focused on the situatedness of communities and its complexity if the research goal is to acquire objective knowledge.

Therefore, feminist epistemology informs the women's personal empowerment conceptual framework in the sense that women in Laos would have better knowledge about their own empowerment due to their social and physical location. Different ethnic women in Laos are associated with their own sociocultural and physical environment, which can determine their own situated knowledges about empowerment. The situated 
knowing approach, in which the reality of individual women's lives is explored, will enable me to identify different factors affecting women in empowering themselves. Women's social and physical location will be taken into account in order to increase my understanding about individual women's views of empowerment. Each woman will be considered as an epistemic agent due to their situated knowledges. This situated knowing approach requires appropriate research methodologies to ensure that objective knowledge is obtained (Grasswick, 2011).

It has been argued that a qualitative approach can enable the collection of in-depth data which reflects the reality of women's lives. In contrast, quantitative methodology is focused on quantitative indicators, and does not reflect the processes through which outcomes are produced, nor the socio-economic and cultural components which have an effect on women's empowerment (Geleta, 2016). Hence this study employs qualitative methodology which is informed by feminist epistemology, and is more capable of acquiring rich knowledge about women's empowerment in Laos.

\subsection{Qualitative methodology}

With a qualitative approach, knowledge is produced based upon the construction of meanings from various perspectives as a result of constructivist or participatory research (Creswell, 2003). Qualitative methodology can be used in various approaches to knowledge inquiry such as ethnographies, case studies, and narratives. This allows researchers to conduct open-ended data collection for the purposes of theme development from emerging data (Creswell, 2003). A qualitative approach is used for an exploratory research purpose where the topic has never been studied or there has not been much research done about it. This approach allows researchers to take into account participants' views, and then construct meanings from the data. Thus, a qualitative approach is employed if there is a need for deeper understanding about a concept or phenomenon without foreknown to-be-studied variables (Creswell, 2003). In addition, a qualitative approach is needed when the variables cannot be measured with numbers. The descriptive nature of this approach is more capable of ensuring justice is given to the complexity of reality (Thiel, 2014).

The use of qualitative methodology in this study means that I listen to my research participants in terms of their experiences and opinions about empowerment. This allows me to delve into the reality of people's lives and be flexible so that I can adjust my research approach and position. I realised that the more attention I paid to individual 
women's stories, the deeper understanding I had about the truth of their lives. This helped increase my confidence in interpreting the meaning of their verbal and non-verbal communication. Some participants just simply kept telling their stories without coming back to my questions. Instead of interrupting them and taking them back to the questions, I encouraged them to finish their stories. This is because I usually positioned myself as a learner, so directing my participants in conversations would prevent me from acquiring important information, and from knowing the truth of their lives.

\subsection{Location, recruitment of participants, and research timeline}

\subsubsection{Research location}

The AFP project was initiated in 2009 with a focus on establishing Village Banks (VB) for the people in rural Laos without access to banks. The project is co-owned by GIZ (a German development agency) and Bank of Laos. By 2014, it was present in six provinces, namely Attapeu, Salavan, Champasak, Savannakhet, Luang Namtha and Sayabouly ${ }^{1}$ (see Figure 3.1). Luang Namtha province, in northern Laos, was chosen as the research location of this study given its involvement in the project (Fuchs, 2014).

Figure 3.1. The AFP project in six provinces of Laos

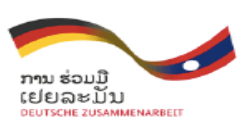

\section{Micro level - Where we are working:}

\begin{tabular}{|l|c|}
\hline Province & Districts \\
\hline Luang Namtha & 3 \\
\hline SAY - Khob & 2 \\
\hline SAY - Hongsa & 4 \\
\hline SVK - Vilabouly & 1 \\
\hline SVK - Sepon/Phin & 2 \\
\hline Salavan & 2 \\
\hline Attapeu & 5 \\
\hline Champassak & 2 \\
\hline Total & 21 \\
\hline
\end{tabular}

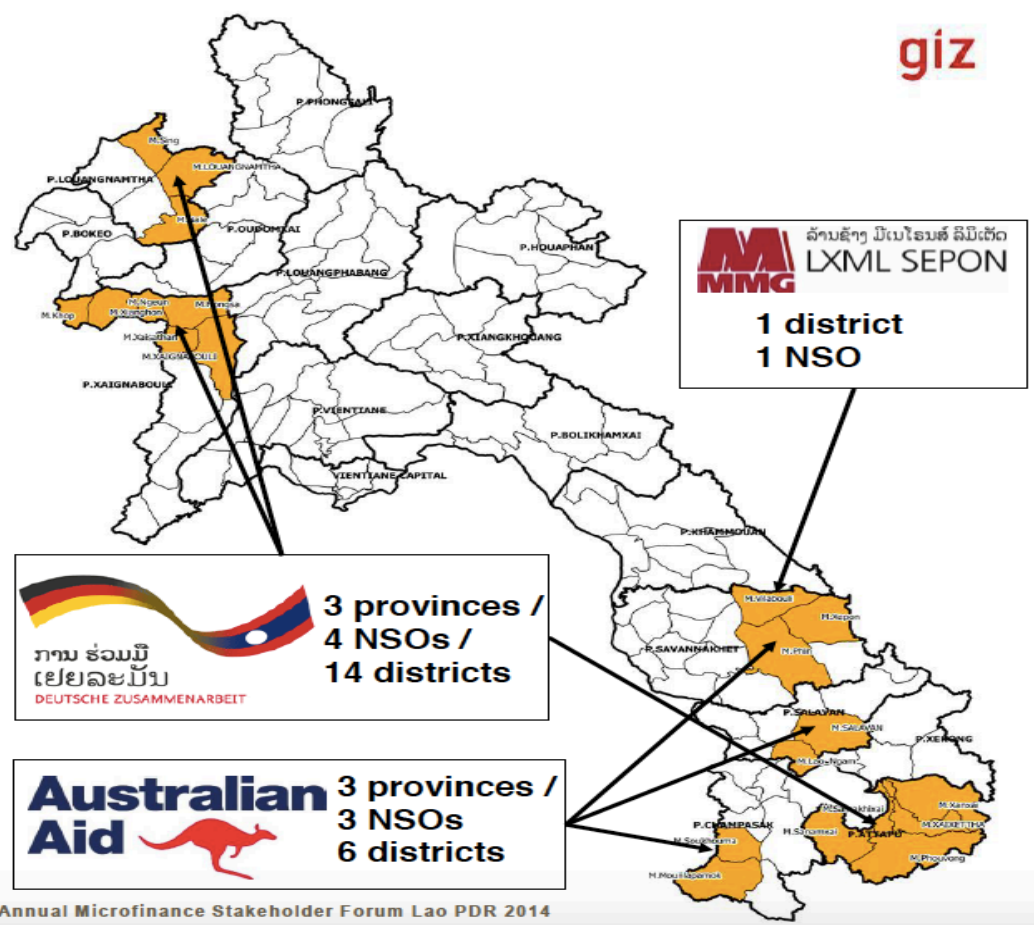

Source: Fuchs (2014), slide 5.

\footnotetext{
${ }^{1}$ Sayabouly and Luang Namtha are two northern provinces; the four southern provinces include Savannakhet, Champasak, Salavan, and Attapeu.
} 
In addition, this multi-ethnic province is where groups of ethnic people participating in the project are commonly found and easily reached. There are more than 20 ethnic groups which form up to 97.7 percent of the population of Luang Namtha province (Luang Namtha Tourism Department [LNTD], n.d.). Akha and Khmu ethnic groups are the majority ethnicities of Luang Namtha's population (see Figure 3.2).

Figure 3.2. Population of Luang Namtha province by ethnicity

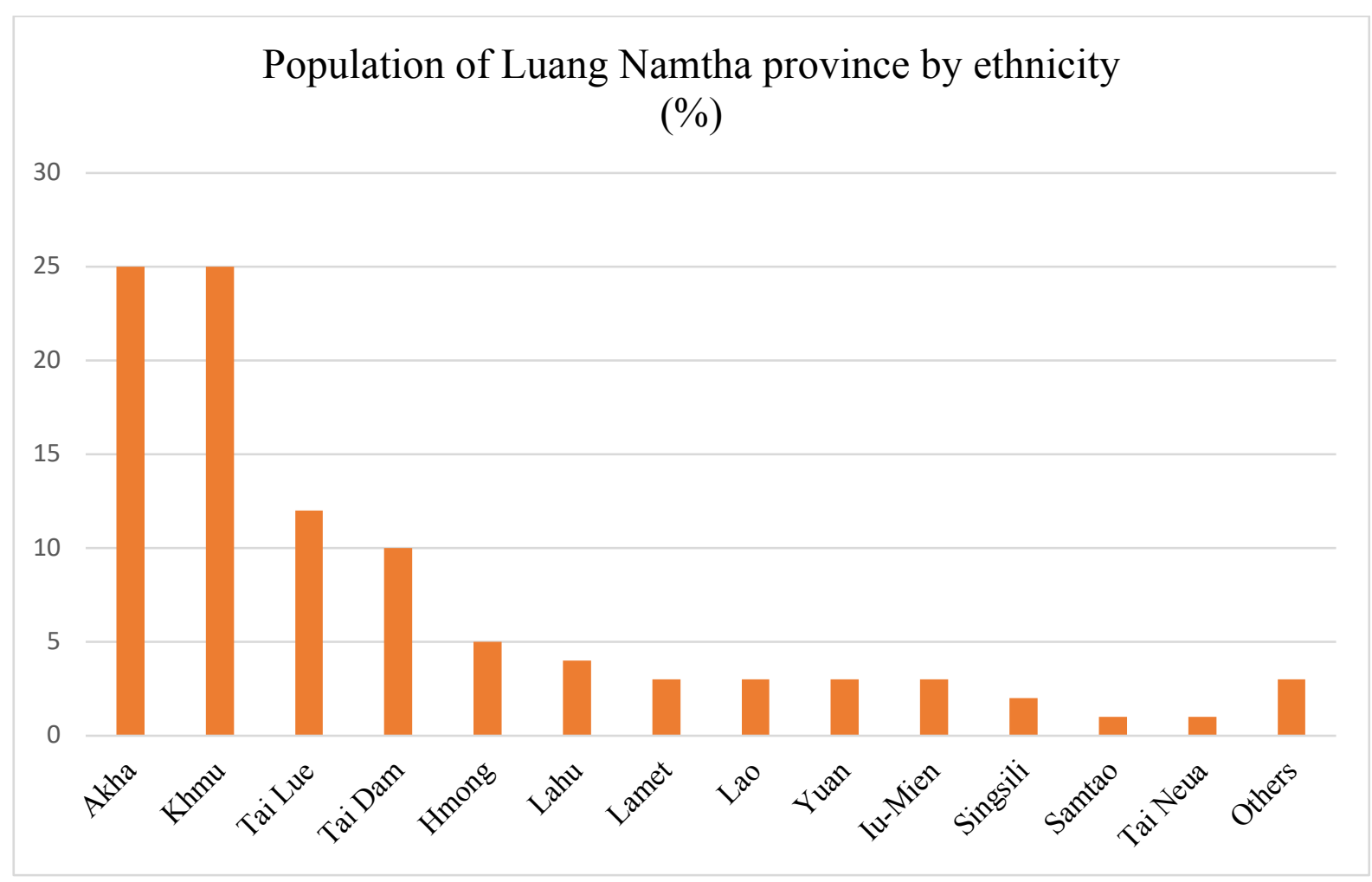

Source: adapted from Government of Laos

(https://www.researchgate.net/figure/Population-of-Luang-Namtha-Province-by-

Ethnicity_tbl1_305409143).

Luang Namtha province is in the north-western corner of Laos (see Figure 4.1). It has an area of 9,325 square kilometres that is mostly covered with low mountains that rise up to 800 to 2,000 metres above sea level. Luang Namtha province has a land border of 140 kilometres with China to the north, and a Mekong River water border of 130 kilometres with Myanmar to the north-west. The province is administratively divided into five districts including Namtha, Nale, Vieng Phoukha, Long, and Sing, and has a total of 380 villages. Luang Namtha's main industries include agriculture, wood processing, lignite and copper mining, handicraft production, transportation and tourism (LNTD, n.d.).

I based my fieldwork in Namtha district. It includes the biggest city of Luang Namtha province, and the most populated city of about 45,000 residents. About 78 percent of 
people in the province are classified as rural, and those aged under 14 years old formed 40 percent of the population in 2005 (LNTD, n.d.). Three villages of the district were targeted based on their ethnic differences as follows:

- Donmoun village is located about ten kilometres to the south of Namtha district centre. This is a Khmu village where I worked with Khmu women.

$\circ$ Viengnuea village is located about six kilometres to the south of the district centre. This is a Tai-Yuan (Yuan) village where I worked with Tai-Yuan women.

- Namdaeng Tai village is located about 25 kilometres from the district centre to the east. This is a Hmong village where I worked with Hmong women.

\subsubsection{Participants}

The main research participants were recruited from women from the three ethnic groups: Tai-Yuan, Khmu, and Hmong. All of the participants are married women, and members of their own Village Bank (VB). Some of them hold a position in the Village Bank Committee (VBC). I changed my recruitment method from systematic sampling to convenience sampling due to the local situation. I was supposed to use systematic sampling in which I would acquire a list of VB members, and then approach the members randomly based on a system of numbering. However, I was faced with the local situation in which people were busily engaged with their farms such as rice growing and rubber tree harvesting. Therefore, I used convenience sampling by which I approached whoever was conveniently available and satisfied my participant criteria, given that using systematic sampling would be time-consuming and put burdens on potential participants.

In addition, two interviews were conducted successfully with two stakeholders of the AFP project in Vientiane, the capital city of Laos. One is from the AFP project of GIZ Laos (GIZ Laos-AFP), and another is from the Financial Institution Supervision Department of the Bank of Laos (FISD-BoL). Unfortunately, Lao Women's Union (LWU) representatives were not interviewed as planned due to their full work schedule. However, I acquired a secondary source of information from the LWU that mostly answers my concerns. Details of the participants are presented in Table 3.1 below. 
Table 3.1. Interview and focus group participants

\begin{tabular}{|c|c|c|c|}
\hline Participant categories & Interviews & Focus groups & Age range \\
\hline Donmoun Village & 6 & 4 & $26-53$ \\
\hline Viengnuea Village & 4 & 6 & $37-52$ \\
\hline Namdaeng Tai Village & 6 & 6 & $28-70$ \\
\hline GIZ Laos-AFP & 1 & 0 & $\mathrm{n} / \mathrm{a}$ \\
\hline FISD-BoL & 1 & 0 & $\mathrm{n} / \mathrm{a}$ \\
\hline
\end{tabular}

Source: Author (2018).

\subsubsection{Timeline}

This research took twelve months to complete. The first three months were spent on preparing the research proposal, the ethics application at VUW, and other fieldwork preparations such as interview questions, advance communication with research locations, and travel arrangements. One month was spent on fieldwork in Vientiane capital city, and on preparing for travel to Luang Namtha province. Another month was spent on fieldwork in Luang Namtha province. The rest of the time was spent on data analysis, thesis writing, and editing.

Figure 3.3. Research timeline

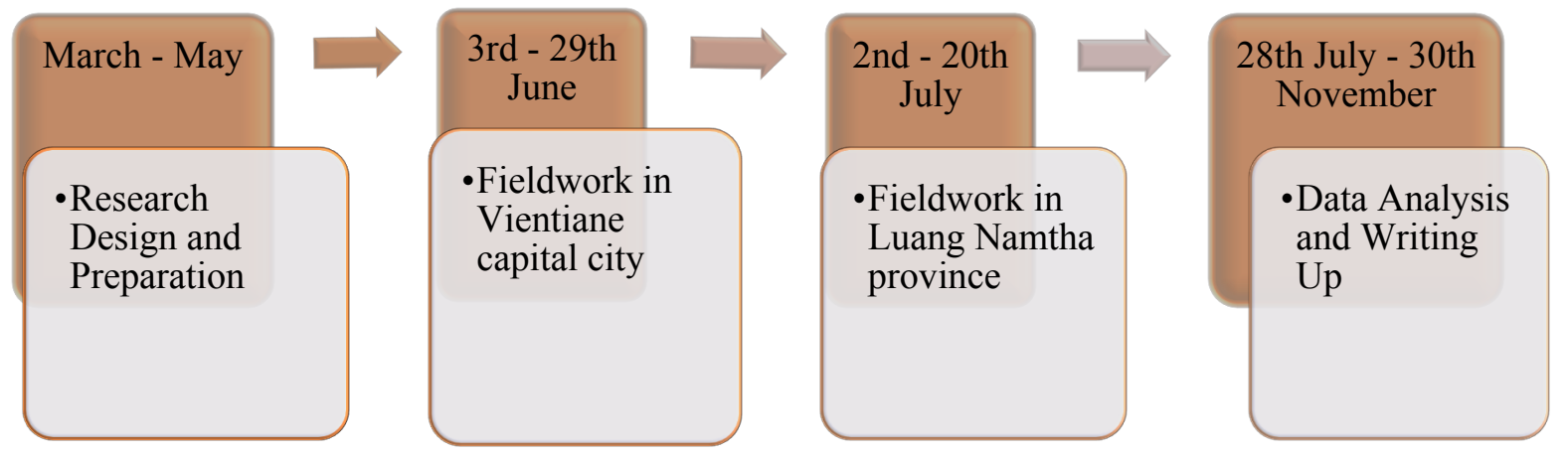

\subsection{Research methods}

The research methods used in this study include semi-structured interviews and focus groups. Each of these methods are explained in detail as follows. 


\subsubsection{Semi-structured interviews}

Semi-structured interviews are a method that links well to a qualitative approach, and are suitable for my research purpose, because they allow the researcher to ask open-ended questions which enable open and fluent conversations between the interviewer and interviewees. This allows the participants to have space for their own interpretation of the questions, and then to form their own answers to the questions from different points of view (Fife, 2005). The interview is normally continued until answers to the researcher's interest are provided (Fife, 2005). Therefore, using semi-structured interviews enabled me to acquire different ethnic women's perceptions of empowerment, and experiences of the AFP project, as well as to begin to understand the women's reality. Interviews help enable researchers to obtain the real meanings of people's experiences (Kvale \& Brinkmann, 2009).

The interviews with female VB members in the three villages took place unofficially and casually. Negotiation regarding interview location sometimes took place between the participants and myself because the majority of participants preferred being interviewed at their home or somewhere with other people present. Therefore, most of the interviews took place at the village office where people felt confident and secure to talk. Some participants came with their family members or friends but that other person or those other people remained at a certain distance from the interview to avoid clearly hearing the discussion. Some interviews were conducted at the participants' house which at times resulted in disruption due to the intervention of family members. There was a case in which I had to pause the voice recorder because the participant was requested by her family members to handle a payment of her household business during the interview.

The interviews with institutional officers happened in a more official manner. Letters of request had been sent to them in advance before the appointments were made possible. Voice recording at some government offices was not allowed. However, the meetings and interviews with the institutional officers took place successfully and productively.

\subsubsection{Focus groups}

In this study, focus groups are used as a complementary method to enrich the data obtained with the individual interviews. As a discussion with a loose structure among a small group of selected people, and with the facilitation of the researcher who normally plans the discussion, the participants' experiences and points of views can be revealed (Litosseliti, 2003). The focus group questions (Appendix 2.5) were designed with some 
overlaps with the interview questions (Appendix 2.1). However, the focus groups were designed to explore mainly the participants' perceptions of empowerment, and the challenges that are facing them. I believed that focus groups would encourage the participants to talk more compared to the individual interviews. From my own experience of the fieldwork, I noticed that more ideas and broader aspects of the participants' lives were explored through the focus groups. The participants were more active and had more initiative to talk as the discussion was led by themselves at times.

In addition, I noticed that the focus groups helped the women gain a sense of security in their participation in the research. They were more confident and willing to join the group discussion as they knew that there were other people like them in the group. There was a case in the Hmong focus group in which a newly married woman wanted to join the discussion but was stopped by her husband. She had already found her seat at the focus group for about five minutes before being asked to leave the group by her husband. Therefore, I found that focus groups encouraged the women, even those who are restricted by male dominance, to gain confidence in taking part in the research. Women want to challenge male resistance even though they understand that the rate of success is very low. Therefore, I saw that even the method itself is an empowering tool for women.

However, both focus groups and individual interviews posed concerns to the participants. They felt worried when I showed them the ethics documents, and even the sheet of interview questions. Instead of creating a feeling of security, showing them the documents caused anxiety and hesitation among the participants. Some of them said they had never done anything like this before so signing or being given a document worried them. Some of the participants declined to sign the consent form but agreed to participate orally.

Given my experience in using the stepping stones diagram (see Appendix 2.6) in a university course, I adapted this method for the focus groups. I placed a large piece of paper in front of the participants with a picture of a river on it to illustrate their journey from where they are to the other side of the river where they wanted to be to achieve their goals. The participants were asked to write what they think they already have in terms of empowerment, and place it on their side of the paper. Then they were asked to write their aspirations of empowerment, and place it on the other side of the paper. This was to help them form a picture of the discussion that followed. After that, the discussion about their reality, aspirations of empowerment, challenges, and enabling factors was started. However, the focus groups were not as effective as expected due to the participants' lack 
of confidence in writing. Particularly, the focus group with the Hmong women resulted in no written form of information due to their lack of confidence in writing. Despite this, the focus group was well recorded in oral form, including those which were supposed to be recorded in written form.

\subsection{Data preparation, analysis, and interpretation}

According to Hammersley and Atkinson (2007), qualitative data can be analysed using either an inductive or a deductive approach. The former means the researcher begins by making sense of the gathered data, then integrates the data into the theory, or comes up with a new theory if the data allows. The latter means the researcher begins by relying on a theory, then uses the collected data to test or prove the theory. Due to the use of the women's personal empowerment analytical framework in this study, the collected data is analysed using an inductive approach. This is because the data from the case studies is used as voices of the local people whose interests are not always the same as that of the policymakers at the macro level. Informed by Creswell (2014), I analysed the data based on the following process (see Figure 3.4).

Figure 3.4. Data analysis process

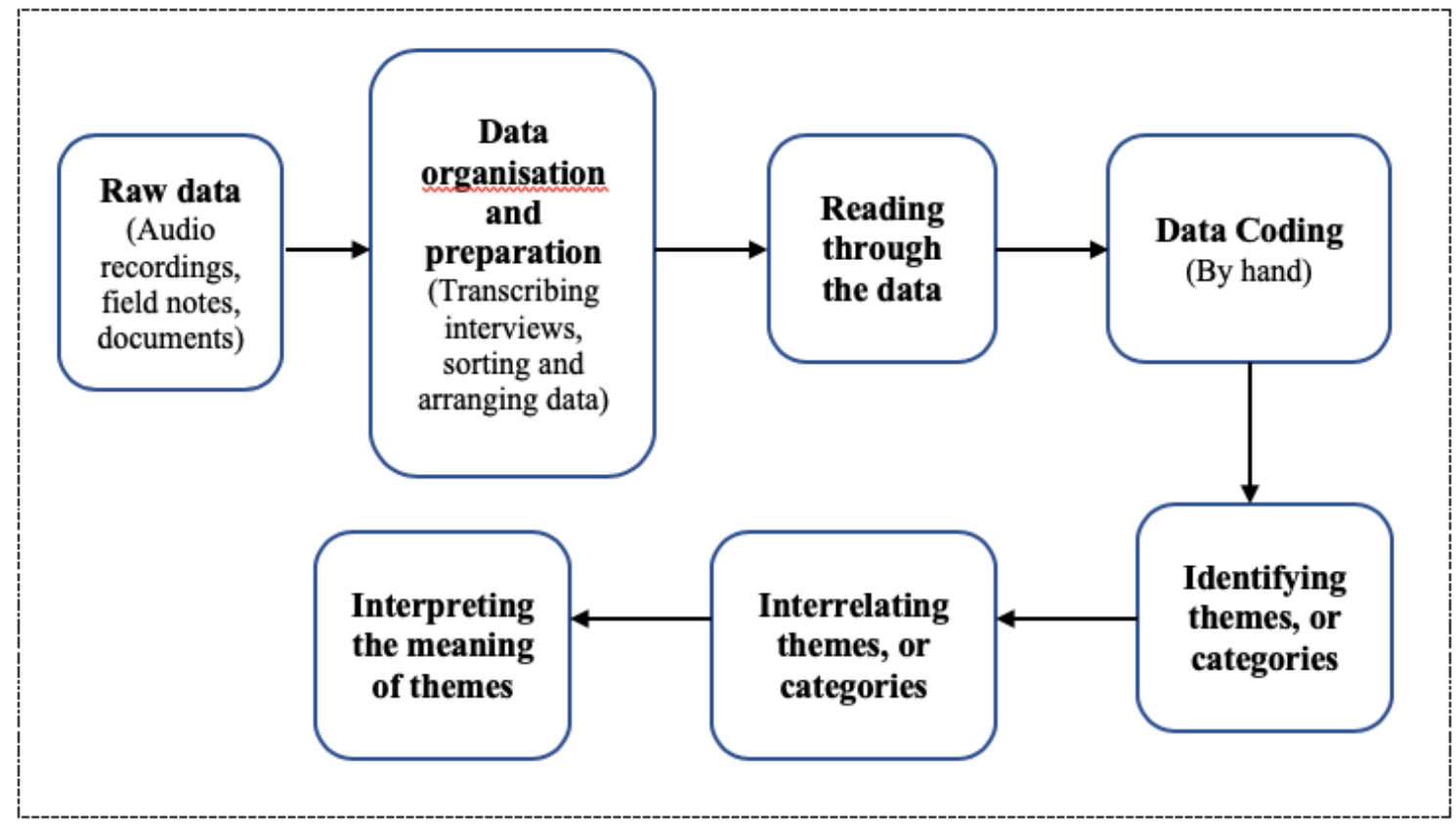

Source: adapted from Creswell (2014, p. 197).

After the data was collected, I started to order them in a systematic manner as suggested by Thiel (2014). Before transcribing, I transferred the audio recordings from the recorder to my computer where they were allocated to different folders in order for them to be transcribed systematically and traced easily. I minimised the time spent on transcribing 
by analysing most of the data, and also generating some ideas, while transcribing (Gibbs, 2007). This was done with a focus on the research questions rather than being distracted by every detail given by the participants. However, any significant expressions, including non-verbal expressions, were taken into account. I was the only one who transcribed the data, as I believe that this option brings about several benefits. It allowed me to start analysing the data, be familiar with the content, and be able to generate ideas in parallel with the transcription process (Gibbs, 2007).

Following the transcription and preliminary data analysis, I read through the transcripts. A read through of the transcripts of data can enable critical thinking and analytical strategies (Crang \& Cook, 2007). Next, I coded the data, and identified themes simultaneously by hand. Themes were formed according to the research questions (Crang $\&$ Cook, 2007). The themes were compared and contrasted to each other; and this enabled patterns in the data to be identified (Braun \& Clarke, 2006). Key themes from the data analysis are shown in Table 3.2 as follows.

Table 3.2. Key themes from the data analysis

\begin{tabular}{|c|c|}
\hline Key themes & Sub-themes \\
\hline Perceptions of empowerment & $\begin{array}{ll}\checkmark & \text { Being proud of oneself } \\
\checkmark & \text { Being helped by men } \\
\checkmark & \text { Being respected by men }\end{array}$ \\
\hline VB's impacts on women & $\begin{array}{ll}\checkmark & \text { Women's family obligations } \\
\checkmark & \text { The different impacts of VBs on VBC members and } \\
& \text { VB ordinary members } \\
\checkmark & \text { Women's mobility } \\
\checkmark & \text { A sense of security } \\
\checkmark & \text { The formation of a savings habit } \\
\checkmark & \text { VB's benefits for the poorest } \\
\checkmark & \text { Hope creation }\end{array}$ \\
\hline Enablers & $\begin{array}{ll}\checkmark & \text { Spiritual practice } \\
\checkmark & \text { Technologies }\end{array}$ \\
\hline Challenges faced by women & $\begin{array}{ll}\checkmark & \text { Poverty } \\
\checkmark & \text { Family obligations } \\
\checkmark & \text { Men's ignorance } \\
\checkmark & \text { The lack of driving skills } \\
\checkmark & \text { Lack of education } \\
\checkmark & \text { Poor health } \\
\checkmark & \text { The lack of markets } \\
\checkmark & \text { The lack of production land } \\
\checkmark & \text { Limited mobility }\end{array}$ \\
\hline
\end{tabular}




$\begin{array}{ll} & \checkmark \text { To be a 'good wife' } \\ & \checkmark \text { To be a 'good mother' } \\ & \checkmark \text { The role of the youngest daughter-in-law } \\ & \checkmark \text { A dropped-out school girl } \\ & \checkmark \text { No way back marriage } \\ & \checkmark \text { 'Women can't do anything' } \\ & \checkmark \text { 'Men are born like that' } \\ & \checkmark \text { The fear of losing face in borrowing } \\ \text { Strategies and interventions } & \checkmark \text { Working hard } \\ & \checkmark \text { Job creation } \\ & \checkmark \text { Regular female meetings } \\ & \checkmark \text { Training for men } \\ & \checkmark \text { Travelling to other cities }\end{array}$

Source: Author (2018).

Another task is data interpretation. According to Willig (2014), there are two approaches in data interpretation: suspicious and empathic approaches. While the former is focused on unravelling a mystery in the phenomenon, the latter is about understanding the phenomenon at its face value. Thus, the suspicious approach is about explanation whereas an empathic stance is about understanding (Willig, 2014). However, the purpose of this research is in-between; it aims at both understanding the people's reality from their point of view, and explaining hidden facts driving their reality. The interpretation with the "explanation orientation" is done based on my ontological and epistemological position (Willig, 2014). The data is interpreted based on a feminist perspective, particularly the conceptual framework used in this study. However, this prescription does not restrict the interpretation in respect of the "understanding orientation" in the sense that the participants' accounts are also taken into consideration at their face value.

\subsection{Ethical considerations}

As a social science researcher, I am highly responsible for my own research. I am committed to being aware of, and being actively engaged in, the research ethics process before, during and after the fieldwork. The nature of semi-structured interviews which are exploratory and open-ended creates many questions regarding the participants' consent, the analysis, interpretation, and presentation of data (Fisher \& Anushko, 2008). Any use of the participants' names in this paper would result in conflicts between them and their male counterparts, given that the interpretation of the data may be against men's interests. Moreover, any interviews of women without the acknowledgment of men and 
relevant authorities would create family violence or concerns regarding the researcher's own safety.

\subsubsection{Entering the field}

Before the fieldwork started, I had obtained ethics approval from the Human Ethics Committee of Victoria University of Wellington (VUW-HEC) (Appendix 4) and a support letter from my supervisor (Appendix 5). As the target participants are members of the AFP project, I submitted a request letter to the owners of the project (Appendix 6.1 and Appendix 6.2). After receiving approval from the project (Appendix 6.3), I started the actual fieldwork immediately.

I was half excited and half worried about starting my fieldwork despite the acknowledgement and approval from the project. The people I was going to meet and work with were totally in my imagination, except for some prior distant contact I had had with two officers of the project in the area. I felt excited in the sense that I would be exposed to the participants' reality in which new insights could be obtained. Meanwhile, I was worried that the people would not be cooperative in order for my data collection to be completed as expected, partly due to their engagement in farming. This urged me to be prepared for an adjustment in my data collection approach in case the local situation would not be as expected.

The approval and support letters from the project rendered two contrasting effects on my data collection. On the one hand, the letters acted as a green light for the VB members to participate in the research. In the introduction of my research to a focus group, a woman raised a question whether the research, particularly my data collection process and technique, had been recognised by relevant authorities including their own village head. They would participate only after they had been allowed to do so with a common understanding of the content of their participation. After I explained the research and presented the support letters from the project, and other relevant documents, to them, they were more confident in taking part in the research. However, the explanation and presentation of the documents did not have the same effect on one of the participants. She decided not to participate by giving her busyness at home as the reason. On the other hand, the project's approval and support letters appeared to affect participants' response to my interview questions. Based on my observation, more than half of the participants appeared to be reserved in responding to my interview questions. The project's support letters changed the participants' image of me from an independent researcher to a project- 
affiliated person. This image may have confused some participants in constructing their responses to my questions. This may be part of what Fisher \& Anushko (2008) talked about regarding conflict of interests in research, and what Yin (2016) noted about the ownership of the data.

All participants including institutional officers were recruited and approached ethically based on the VUW-HEC guidelines. The majority of participants were happy to sign the consent forms, whereas a minority of them preferred to give their consent orally. To ensure that the participants with any barriers such as language, fear of exploitation, and male dominance (as noted by Marshall, 2003) could give informed consent, I explained every condition in the consent form to them. All oral consents were audio recorded, and securely kept together with the other types of documents and data. In conforming to the ethics approval, no photos of the participants or their properties were taken. Their names were recorded on the consent forms and interview schedules, but there is no use of those names in the presentation of data.

Despite the challenges facing me in the fieldwork, the data was collected on a basis of mutual trust and understanding. Establishing relationships of trust with the research participants is very important as suggested by Fisher and Anushko (2008). It is also noted that "openness and honesty are indicators of integrity and responsibility, and characteristics that promote quality research as well as strengthen the research process" (Fisher \& Anushko, 2008, p. 99). Therefore, almost interviews and discussions took place in a friendly and relaxed manner. The purpose of my research was clear to the participants, and thus incentivised them to reveal their reality. Some VB members even placed their expectations on me to bring about changes to the community. Some even asked me to give them suggestions to help deal with their personal problems. Some AFP project officers expressed a desire to receive my thesis after its completion. The project owners, known as GIZ Laos and Bank of Laos, expected to have a presentation given by me after the completion of data analysis. With the goodwill of the research participants and the expectations of people on the ground, I was well supported throughout, and even after the fieldwork.

\subsubsection{Leaving the field}

Being prepared for exiting the field is just as important as getting ready for entering the field (Yin, 2016). This is because in most cases the researcher does not have a chance to return to the location. Therefore, the leaving of the researcher needs to mean mutual 
understandings between the researched and researcher have been achieved. However, there is no particular strategy for leaving the field given that it depends mainly on the relationships built during the fieldwork (Yin, 2016). I managed to exit the field by revisiting the data collected to ensure a theoretical grasp of the data (Snow, 1980). I also had a chance to say goodbye to the AFP project office in Luang Namtha province in person. Summaries of interviews were given to those participants who requested it. For those I could not meet in person, I made phone calls and contacted them by email.

\subsubsection{Interpreting the meanings and use of language}

Most of the interviews and discussions were conducted in Lao language, except those with the Hmong women who I worked with in Hmong. Both data collected in Lao and Hmong were translated into English by myself. The data was translated with much care taken to maintain its original meanings and the emotion of the participants. I take full responsibility for any error in the translation.

\subsection{Positionality}

I am a male researcher studying women, who has Hmong ethnic origin, who speaks both Hmong and Lao, and who comes from the same country as the research participants. I have much knowledge about the Hmong ethnic group but know little about the other ethnic groups, particularly the Tai-Yuan group. These variations between me and each of the participant groups rendered different positional distances between me and each of the ethnic groups of women.

For the Tai-Yuan women, I was considered more of an outsider. I felt less comfortable as I did not have much information about the ethnic group, particularly the women. My cultural background, languages, education, and gender appeared to have a significant influence on the interviews. However, such binaries were reduced to some extent with my expression of interest in women's poverty and realities. An example of this was when a Tai-Yuan woman said with delight, "You think exactly like me!", when talking about women's poverty.

In contrast, I was considered more of an insider by the Hmong women. This was due to my similar cultural background. The women were excited and happy that I spoke their language. Some told me they were happy because they have not seen a lot of Hmong being educated abroad, particularly at western universities. They even invited me to have a stay in the village overnight. Their hospitality almost removed the 'me' and 'them', 
except what was rendered by the differences in gender and educational background. My gender difference and educational background brought about reluctance and selfdeprecation among the women in participating in the research. This was seen when most women did not feel comfortable sitting close to the discussion table. Some did not want to write because they did not want me to see their handwriting. They generally thought that I was well educated, and expected that I would be able to bring about changes in their community. These dynamics made me feel like an outsider.

Unlike these two ethnic groups, I felt like I was considered as an outsider and insider in equal measure by the Khmu women. I had some basic knowledge about the Khmu community so that I did not feel any significant divide between me and them. A woman even called me 'son' when talking to me. I used 'our' rather than 'your' language in talking and referring to them and their lives. This made me feel like an insider. However, I was seen as an outsider due to my ethnic and educational backgrounds. In talking to the women in the focus group, they at times compared Hmong and Khmu cultures with a reference to my cultural difference. Some asked me about my life in New Zealand and the country in general. They thought I was very privileged to be allowed to study in a developed country. This made me feel like an outsider.

In working with institutional officers, I was viewed as an insider when the AFP project expected me to share my findings with them. They noted that my research could be a project evaluation similar to what they were about to do in southern Laos. This brought about their strong support for my research. This made me feel like a colleague rather than an outsider. However, I was considered as an outsider when the project owners voiced, at the meeting between us, that the enabled fieldwork was due to their cooperation; therefore, it was a "no free lunch" research output.

\subsection{Reflexivity}

With a Hmong background, and having one younger brother and three sisters, I have witnessed countless acts of gender-based discrimination and discriminatory behaviour in my community, as well as within my own family, since an early age. I have witnessed several girls being pulled in tears by men to be wives, family conflicts and violence due to polygynous marriage, girls dropping out of school for family obligations and their own brothers' education, early marriage, girls being sold for a sack of money because of family poverty, and more. I used to blame my own sisters, who dropped out of school to marry and now live in misery, for not having worked hard on their education. As I grew up 
physically and educationally, I started to question many of these gender-based phenomena. I have become suspicious about, and interested in, the hidden facts driving so many gender-based differences and this discriminatory treatment.

Therefore, I acknowledge that the process and outcomes of this study are inevitably influenced by my previous background and prior knowledge. These experiences and assumptions have a significant effect on how and what data is collected, and on how data is analysed and interpreted. The meaning given to the data says more about myself rather than the research participants (Willig, 2014). Moreover, the ownership of data may also be questioned due to the multiplicity of stakeholders of the research. I also recognise that my gender imposed some hindrances to the research process, due to the fact that men usually have more power than women because men tend to be more advantaged than women structurally and externally (Carli, 1999). The power differences between the participants and myself meant that the women had a less direct manner in our interactions. This affects the data collection and interpretation. Despite all potential consequences as a result of my positionality, it is argued that knowledge can be achieved at the crossroads between the interpreter and the data itself (Gadamer, 2004; Schmidt, 2006).

\subsection{Summary}

In this chapter, I explained the study's epistemology as the philosophical stance of this research. I also elaborated on the data collection methods and research location. The fieldwork and data analysis process were clarified. The chapter also included my reflection on the research process. However, before being able to provide answers to the research questions, there is a need for an understanding of the national context of Laos, which is the subject of the following chapter. 


\section{Chapter 4: The social, economic, and political contexts of Laos}

\subsection{Introduction}

This chapter focuses on exploring the social, political, and economic contexts of Laos. The first section of this chapter is focused on introducing the physical location, and exploring Laos' people, ethnicity and culture. It particularly focuses on the three ethnic groups of the three case studies of this research: Tai-Yuan, Khmu, and Hmong. The second section of this chapter aims to provide some insights in respect of Laos' political and economic situation. The chapter ends with an exploration of the Village Bank project and its development. Three particular village banks in Luangnamtha province are also introduced in this section.

\subsection{Geography, people and culture}

Lao PDR is a relatively small country bordering with China, Vietnam, Cambodia, Thailand, and Myanmar. Its total land area is 236,800 square kilometres (United Nations, 2015). Most of the country's land area (about 80 percent) is hilly and mountainous causing difficulties in accessing remote areas. The majority of the population is concentrated in lowland areas which form about 20 percent of the country's area. In regard to administration, the country consists of 17 provinces $^{2}$ and one capital city. These administrative divisions consist of 145 districts and 8,600 villages (Government of the Lao PDR, 2014, as cited in United Nations, 2015).

\footnotetext{
${ }^{2}$ Xaysomboun was officially designated as a province in December 13, 2013, upon National Assembly Decree No. 012/NA, after having been a special administrative zone under the administration of Vientiane province for decades (Lao Statistics Bureau, 2016).
} 
Figure 4.1. Geographic location of Laos

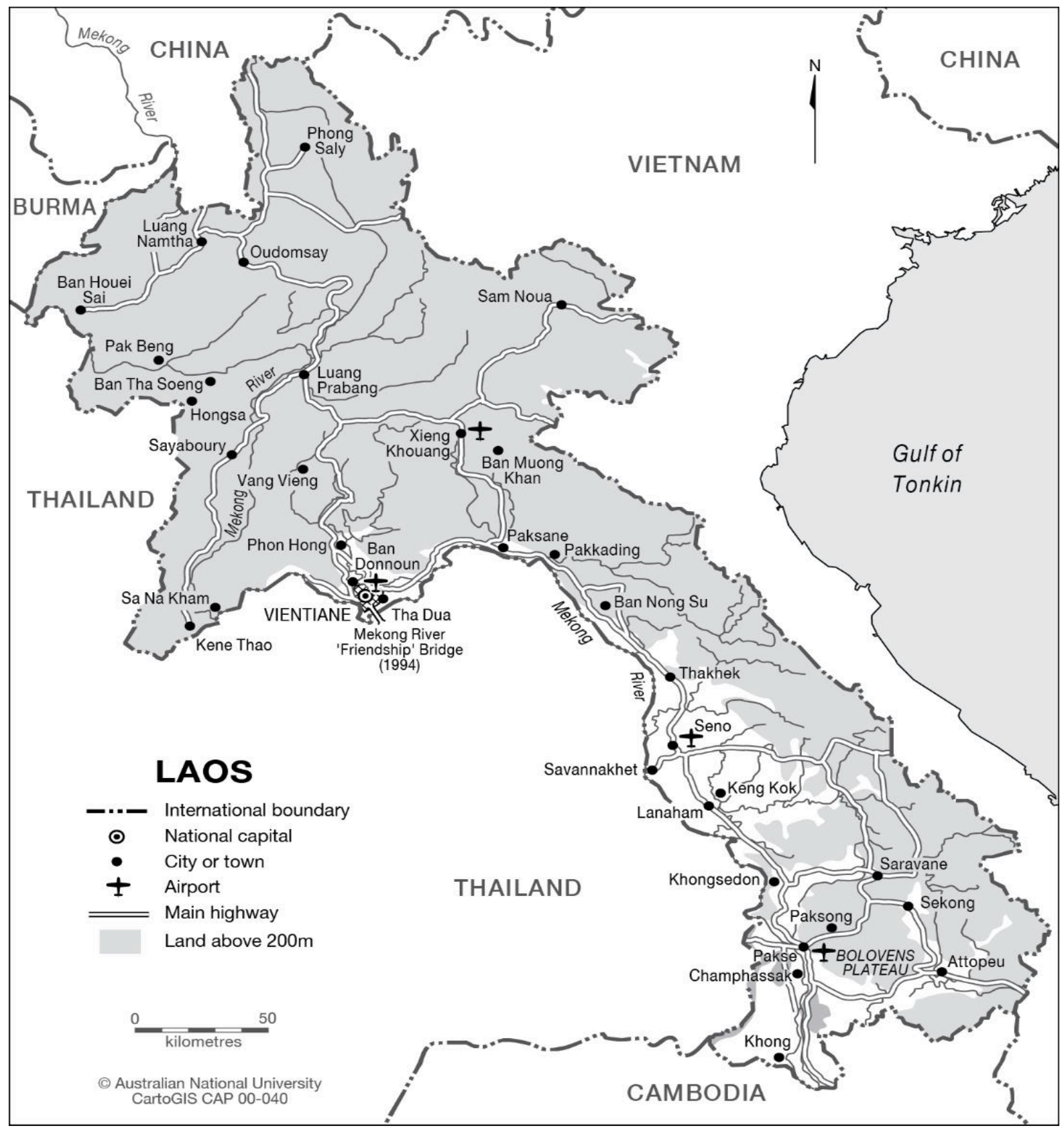

Source: Australian National University (n.d.).

http://asiapacific.anu.edu.au/mapsonline/base-maps/laos

Lao PDR had a population of about 6.8 million people in 2012 (United Nations, 2015).

The proportions of males and females are relatively equal (Lao Statistics Bureau, 2016).

It is a young population with more than half the population aged under 25 in 2015. Both male and female have similar life expectancy at 65.8 years (United Nations, 2015). Most of the population (67.1 percent in 2015) reside in rural areas. This number of people is declining at an estimated rate of 9 percent per year due to rural-to-urban migration, particularly to the capital, Vientiane (Lao Statistics Bureau, 2016). In addition, there is a trend of migration to Thailand for work, given that the two countries share similar 
linguistic backgrounds. However, there is a lack of reliable information about this movement (United Nations, 2015).

Lao PDR has multiple ethnic groups with different dialects, traditions and cultures. There are 49 ethnic groups and hundreds of subgroups. Due to linguistic similarities and differences, these ethnic groups are categorised into four main groups, namely Lao-Tai, Mon-Khmer, Hmong-Iu Mien and Chinese-Tibetan (see Figure 4.2). The Lao-Tai is the official language of Laos, but it is not spoken among many ethnic groups (United Nations, 2015).

Figure 4.2. Ethnolinguistic groups of Laos

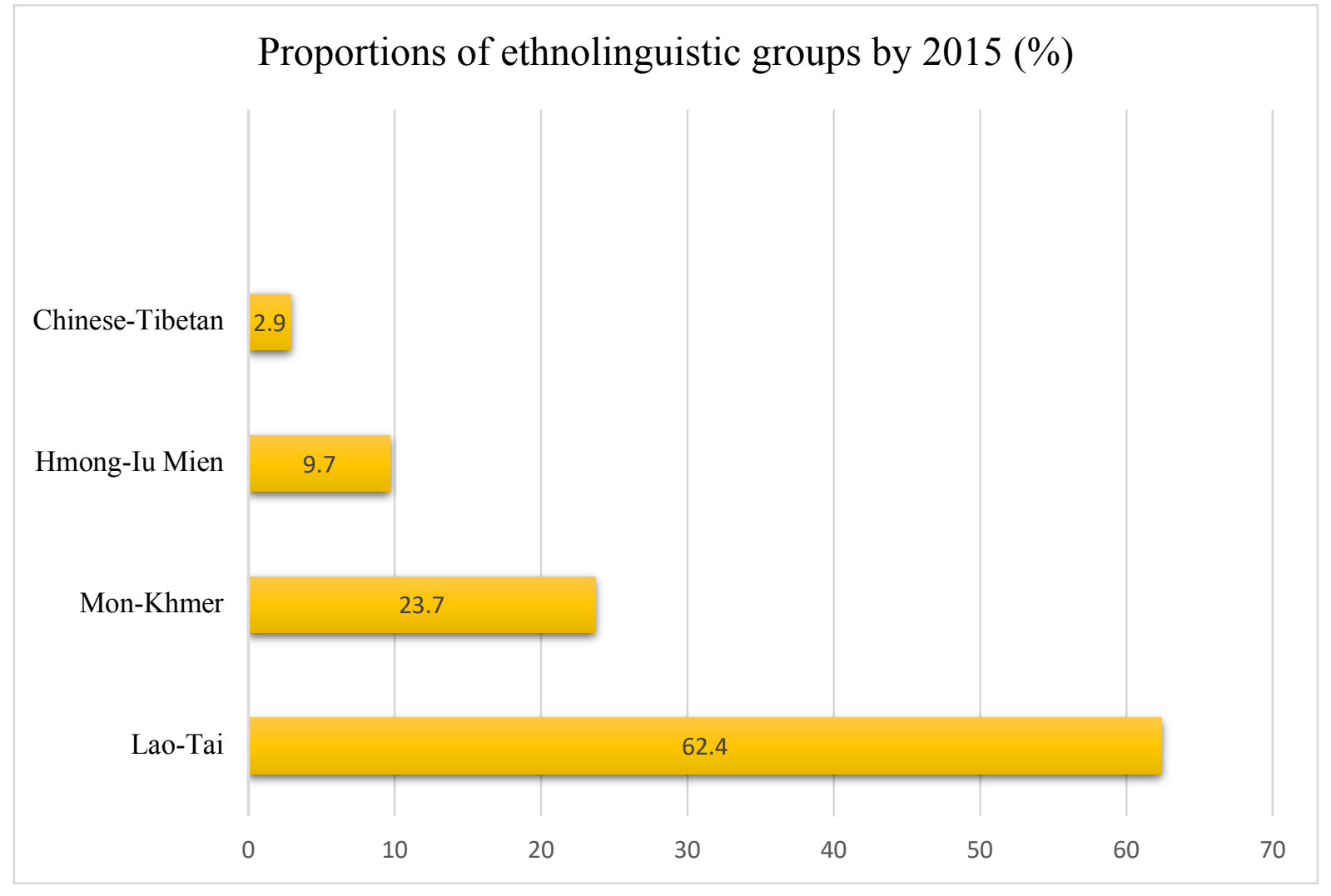

Source: adapted from Lao Statistics Bureau (2016, p. 9).

The Lao-Tai family is comprised of eight ethnic groups; the Mon-Khmer of 32 groups; the Hmong-Iu Mien of two groups; and the Chinese-Tibetan of seven groups (Lao Front for National Construction [LFNC], 2008). These ethnic groups are listed in Table 4.1 below. 
Table 4.1. Ethic groups of four ethnolinguistic families

\begin{tabular}{|c|c|c|c|c|c|c|}
\hline Lao-Tai & \multicolumn{4}{|c|}{ Mon-Khmer } & Hmong- & Chinese- \\
\hline Saek & Khmu & Samtao & Toum & Moy & Hmong & Singsily \\
\hline Yuan & Katang & Sadang & Thaen & Yrou & Iu Mien & Sila \\
\hline Tai & Katu & Xuay & Bid & Yae & & Lahu \\
\hline $\begin{array}{l}\text { Thai } \\
\text { Neua }\end{array}$ & Kriang & Xingmoun & Brao & Lamed & & Lolo \\
\hline $\begin{array}{l}\text { Phou } \\
\text { Thai }\end{array}$ & Kree & Nhahuen & Pakoh & Lavi & & Hor \\
\hline Yang & Khmer & Ta-oy & Pry & $\mathrm{Oy}$ & & Akha \\
\hline Lao & Ngouan & Triang & Phong & Oedou & & Hanyi \\
\hline Lue & Cheng & Tri & Makong & Harak & & \\
\hline
\end{tabular}

Source: adapted from LFNC (2008).

This study targets three ethnic groups (highlighted in green): Yuan (Tai-Yuan) from the Lao-Tai family group, Khmu from the Mon-Khmer group, and Hmong from the HmongIu Mien group. These ethnic groups are explored in more detail as follows.

\section{a) Tai-Yuan ethnic group}

The Tai-Yuan ethnic group is in the Lao-Tai family. The Tai-Yuan people are believed to have migrated from the northern part of the Mekong River in southern China to northern Laos in the thirteenth century (LFNC, 2008). The Tai-Yuan normally reside in the lower plains and close to rivers in order to enable easier farming. This ethnic group is comprised of two subgroups or twigs: Kalrom and Ngeu. The Tai-Yuan ethnic group has a total population of about 26 thousand people forming about 0.6 percent of the total population of Laos. More than half of them are women. This ethnic group adopted Buddhism as their dominant spiritual belief about ten centuries ago. However, they also maintain the practice of animism (LFNC, 2008).

Traditionally, the Tai-Yuan group is patrilineal but matrilocal to some extent. This ethnic group follows a monogamous marriage tradition. The family is headed by the husband and follow the father's line. However, males normally move to live with their wives and parents-in-law for certain years after marriage. After that, the couple can return to the men's family, or move out to have their own house (LFNC, 2008). Men and women have 
freedom in choosing their own partners. However, the woman's family is the one who asks the man's family to arrange a marriage of their children. If the man agrees, a marriage date will be arranged.

Schenk-Sandbergen (2012) conducted a study on the matrilocal and matrilineal culture of the Lao ethnic group, which is also shared with the Lao-Tai ethnolinguistic family group, and shares several similarities with the Tai-Yuan ethnic group. The study shows that the matrilineal system works in favour of the daughters, particularly the ones who take care of the elders, in such a way that family properties are inherited by daughters. The Tai-Yuan ethnic group is not matrilineal like the Lao ethnic group, but the matrilocality of the Tai-Yuan enables very limited discrimination in property inheritance between daughters and sons.

\section{b) Khmu ethnic group}

The Khmu ethnic group is in the Mon-Khmer family. Mon-Khmer are believed to have been the first people to appear in southeast Asia, including Laos, five thousand years ago or more (Chamberlain, 1995, as cited in Inui, 2015). The Khmu ethnic group is comprised of nine subgroups: Kasak, Khmu Ou, Khmu Lue, Khmu Nhouan, Khmu Khrong, Khmu Rork, Khmu Kwaen, Khmu Mae, and Khmu Cheung (Anonymous, 2000). These Khmu clans form the second largest population of Laos, following the Lao ethnic majority (LFNC, 2008). These people mainly reside in mountainous areas in northern and central regions of Laos (LFNC, 2008), and have several religious practices such as Buddhism, animism, and other religions (Inui, 2015).

Traditionally, the Khmu ethnic group follows patrilocal and patrilineal systems (SchenkSandbergen, 2012). Generally, females move to live with their husbands and parents-inlaw after marriage, but there are cases in which males may have to move to their parentsin-law's family permanently, if the family is economically poor (Schenk-Sandbergen, 2012). There are also cases in which males, usually the poor, have to pay off the high bride price with labour, by staying with their in-laws for a few years after marriage (Schenk-Sandbergen, 2012). Khmu children adopt their father's totem; and men act as the organisers and leaders of major traditional ceremonies of the group (Premsrirat, 2002). Men and women share some responsibilities, such as looking after the house, raising children, working in the field, and fishing. However, women are usually responsible for household work and cooking preparations. They particularly contribute to crafting activities such as making mats, pillows, and some musical instruments from bamboo, while men mainly do hunting (Premsrirat, 2002). 


\section{c) Hmong ethnic group}

The Hmong ethnic group is in the Hmong-Iu Mien ethnolinguistic family, which includes only two ethnic groups: Hmong and Iu Mien (Anonymous, 2000). Hmong is comprised of three subgroups known as Hmong Khao (White Hmong), Hmong Khiao (Green Hmong), and Hmong Dam (Black Hmong), based primarily on their own clothing characteristics and language variations (Anonymous, 2000). Hmong are believed to have been present in Laos in the mid nineteenth century. The ethnic group has its origin in Tibet, China from where the group started to migrate down to northern Vietnam, Laos, and Thailand due to wars (LFNC, 2008). The Hmong population in Laos is now centred in the northern regions of the country, down to Borlikhamxay province, and forms the third largest ethnic group (9.2 percent) in Laos, following the Lao and Khmu (Lao Statistics Bureau, 2016, p. 37).

In terms of religion, the Hmong community practises a mixture of animism, naturalism, and spirituality (LFNC, 2008). The Hmong have a strong belief that all sorts of spirits are everywhere, including the spirits of the deceased, and the spirits of the house, village, region, sky, forest, rivers, and many others. These spirits are worshiped, primarily to gain their protection and support. The Hmong strongly believe that the spirits of the deceased male figures are always in the house, particularly at the central pillar of the house (LFNC, 2008).

Traditionally, the Hmong follow patrilocal and patrilineal systems (Inui, 2015). The male bloodline and clan are followed invariably for distinguishing and tracing the Hmong people (LFNC, 2008). The Hmong group is comprised of 19 clans in which marriage within the same clan is prohibited. After marriage, females move to live with their husbands and in-laws, and adopt the beliefs and genealogy of their new family. By doing this, the Hmong believe that the new member (daughter-in-law) will be protected by the spirits of her in-laws' family, given that her marriage terminates the protection and support of the spirits of her birth clan. Her stepping out of her birth parents' family is considered as permanent. A daughter, whether married or not, is not allowed to give birth in her parents' house or the house of any other clan other than her husband's, given that the house spirits of her birth parents' home or those of others' will get offended, and this will lead to bad luck and disasters for her parents' or the other clans' families (LFNC, 2008).

In Hmong tradition, women continue to take a disproportionately big role in the family (Inui, 2015). Commercial and economic activities are mainly undertaken by women; 
however, the proportion of resources allocated to women is still smaller than that of men (Than \& Tan, 1997). Women mainly undertake household responsibilities such as child raising, food preparation, and farming. However, women are usually secondary people in the household; for example, women always eat after men in the case of ceremonies or having guests in the house. Because clan distinction is very strong in the Hmong culture, the woman will remain in her in-laws' clan after divorce or after her husband's death until she remarries (CLFNC, 2017).

\subsection{Political system and socioeconomic development}

This section overviews the political system and socioeconomic development in Laos. It also explores the country's progress on gender inequality reduction. This involves its policies towards gender issues and human rights. The background knowledge in this chapter will help us understand the situation of gender empowerment which will be investigated in the following chapters.

\subsubsection{Political system}

Lao PDR has been ruled by the Lao People's Revolutionary Party (LPRP) since the country's independence in December 1975 (Freeman, 2001). Like some of its neighbouring countries, such as the People's Republic of China and the Socialist Republic of Vietnam, Laos is ruled by a unitary party which follows Marxist-Leninist ideological orthodoxy (St. John, 1995; Stuart-Fox, 2007). The LPRP is declared to be "the sole faithful representative of the interests of the working classes, the working people of all Lao nationalities [ethnic groups] and the entire Lao people" (Kaison Phomvihan, 1977, as cited in Stuart-Fox, 2002, p. 103). According to articles 1, 2 and 3 of the Constitution of the Lao People's Democratic Republic, all ethnicities are the owners of the country, and have all powers and rights which are ensured through the unitary leading of the LPRP. Article 8 of the Constitution states that "all ethnic groups have the right to protect, preserve, and promote the fine customs and cultures of their own tribes and of the nation" (Lao PDR, 1991).

The administrative structure of the country is comprised of the National Assembly, the President of state, the government, the local administration, and the judiciary organisations (Lao PDR, 1991). The National Assembly is formed with representatives of the people. It is the legislative organisation which "has the right to make decisions on [the] fundamental issues of the country, [and] supervises and oversees the activities of 
the administrative and judicial organisations", according to article 39 of the Constitution (Lao PDR, 1991). The President of state “is the Head of state of the Lao People's Democratic Republic and the representative of the multi-ethnic Lao people both at home and abroad", according to article 52 of the Constitution. The government "is the administrative organisation of the state, and manages in a unified manner the implementation of duties of state in all fields: political, economic, cultural, social, national defence and security, and foreign affairs", according to article 56 of the Constitution. It has been seen that power remains in the hands of the Party due to the overlapping of membership between the Party and government members (Stuart-Fox, 2002). Local administration is at the level of provinces, municipalities, districts and villages, according to article 62 of the Constitution. Finally, the judiciary organisations include the People's Courts and the Public Prosecution Institutes (Lao PDR, 1991).

Following its independence in 1975, Lao PDR was determined to build its country towards socialism without bypassing capitalism by simultaneously undertaking three revolutions. These included production; scientific and technological; and cultural and ideological revolutions. The three revolutions were believed to enable the country to construct socialism, given that the first revolution, in production, acted as a guiding direction for Laos' development; while the second, scientific and technological, was believed to bring about changes in economic development; and the third, cultural and ideological, was concerned with ensuring the other two were happening under MarxistLeninist ideology (Stuart-Fox, 2002). The revolution in production, which led to agricultural co-operativisation, failed to gain people's support, and started to create dissatisfaction and resistance. Therefore, in the mid-1980s, the LPRP decided to abandon the revolution, and undertake an economic reform (known as the New Economic Mechanism or NEM); which is further articulated in the following subsection, followed by its gendered implications. Despite the abandonment of agricultural co-operativisation, the LPRP continues to direct Laos' development due to national security concerns and ideological commitments (Stuart-Fox, 2002).

\subsubsection{Economic reform and its effects on women}

National and international contexts determined the economic reform in Laos. On the one hand, Lao people, particularly farmers and those who had been under the royalist administration which had been supported by America during the Vietnam war, increasingly resisted the state agricultural co-operativisation programme. The growing dissatisfaction among people, and the failure of the cooperative programme, encouraged 
a flow of Lao, including ethnic people, to travel to northeast Thailand for refuge. This movement was believed to cause security concerns for, and pose political uncertainties to, the newly founded Party and State. On the other hand, the deteriorating Soviet Union, which collapsed in 1991, at that time weakened the support from socialist countries to LPDR. These two external and internal situations urged the LPRP to adopt a "new thinking" (chintanakan mai) known as the "New Economic Mechanism-NEM" in the late 1980s (Stuart-Fox, 2002, p. 196). This marked the start of economic liberalisation in Laos. Following the policy change, the Lao economy was multisectoral and embarked on moving from a natural economic system to a market economy, according to Article 13 of Laos' Constitution (Lao PDR, 1991). It is also stated in Article 14 of the Constitution that "private ownership [by] domestic capitalists and foreigners" is protected by the State. The policy changes again enabled Laos to receive international aid, and attract foreign investment (Stuart-Fox, 2002). According to Stuart-Fox (2002, p. 300), the state role in the economy has shrunk from "managing" to "adjusting".

There have been studies showing that the economic reform has changed Lao women's lives considerably. Ireson-Doolittle and Moreno-Black (2004) contend that the economic liberalisation brought about both opportunities and challenges to Lao women. Prior to the NEM, women were reliant on the distribution system of the collective mechanism. This mechanism ensured women's equal participation in and benefits from the system. However, women lacked opportunities for self-development. In contrast, the economic liberalisation provided women with business opportunities, and freedom of spiritual belief. Lao women, particularly Lao ethnic majority women who live in urban areas, are increasingly exposed to business activities, like producing textile and crafted products which are demanded by foreign markets. Despite these advantages, women after economic liberalisation face employment competition in which many women are disadvantaged and excluded (Ireson-Doolittle \& Moreno-Black, 2004). Women constitute a smaller share ( 35 percent) than men in wage employment across all sectors (United Nations, 2015). In addition, women form up to 65 percent of unpaid workers in families. Low education among women contributes to them being disadvantaged in wage employment and in the family (United Nations, 2015).

Moreover, women normally hold lower positions in the labour market (United Nations, 2015). More than 60 percent of women work in the informal sectors including shops, market sales, and other elementary work such as cleaning and agriculture. In contrast, men generally occupy positions in the formal sector, including public mass organisations, 
major companies, and other professional offices. It is found that only 23 percent of women are employers of small enterprises; and they are generally not employers in medium and large companies (United Nations, 2015). It is also argued by the United Nations that women's wellbeing is compromised by their commitment to multiple roles, by which women need to work at an earlier age. Girls are found more economically active than boys across all ages and locations. Women are estimated to work four times longer than men each day due to their additional housework chores. The percentage of urban working girls aged between 15 to 19 is much higher (40 percent) than that of urban boys (28 percent) of the same age. This is concluded by the United Nations to be a factor for women's low enrolment in secondary and tertiary education, and as perpetuating underage marriage among girls in remote areas, particularly.

In addition, the United Nations (2015) also reported that women have become more at risk with their migration for work. The increase in rural-urban migration of women is generally to meet the labour demand of several growing industries, including the exportoriented garment industry. While working in these industries, women are normally found to be faced with several employment disadvantages and issues, including working in poor conditions, underpayment, working long hours, violence, verbal abuse, and penalties. For uneducated women, sexual abuse, exploitation, unwanted pregnancies and unsafe abortions are common. Those who migrate for work abroad, normally in the country's neighbouring countries like Thailand, also face similar problems. They normally migrate at an earlier age (under 16 years of age) than men (21 years of age). Therefore, they are more likely to encounter the risks of being abused and exploited. It is also noted that women from resettled areas constitute a remarkable percentage of the victims of human trafficking. These challenges faced by women can be further comprehended in the following subsection about economic performance.

\subsubsection{Economic growth and gender equality}

The Lao economy is based primarily on natural resources (World Bank, 2018). About one third of economic growth is attributed to hydropower, minerals, and forests. However, the report on the implementation of the seventh National Socio-Economic Development Plan (NSEDP) (2011-2015) shows that the country has achieved some significant development outcomes. Its Gross Domestic Product (GDP) grew at an average of 7.8 percent over the last decade. The country's strong economic growth over the past few years enabled the income per capita to reach USD2,330 in 2017 compared to USD1,600 in 2014 (United Nations, 2015). Laos' economic structure has experienced 
some changes in the last decade (see Figure 4.3). Both the industry and the services sectors have increased whereas the agriculture-forestry sector has shrunk steadily since 2010. The services sector is the biggest sector of the economy.

Figure 4.3. Economic structure between 2010 and 2015

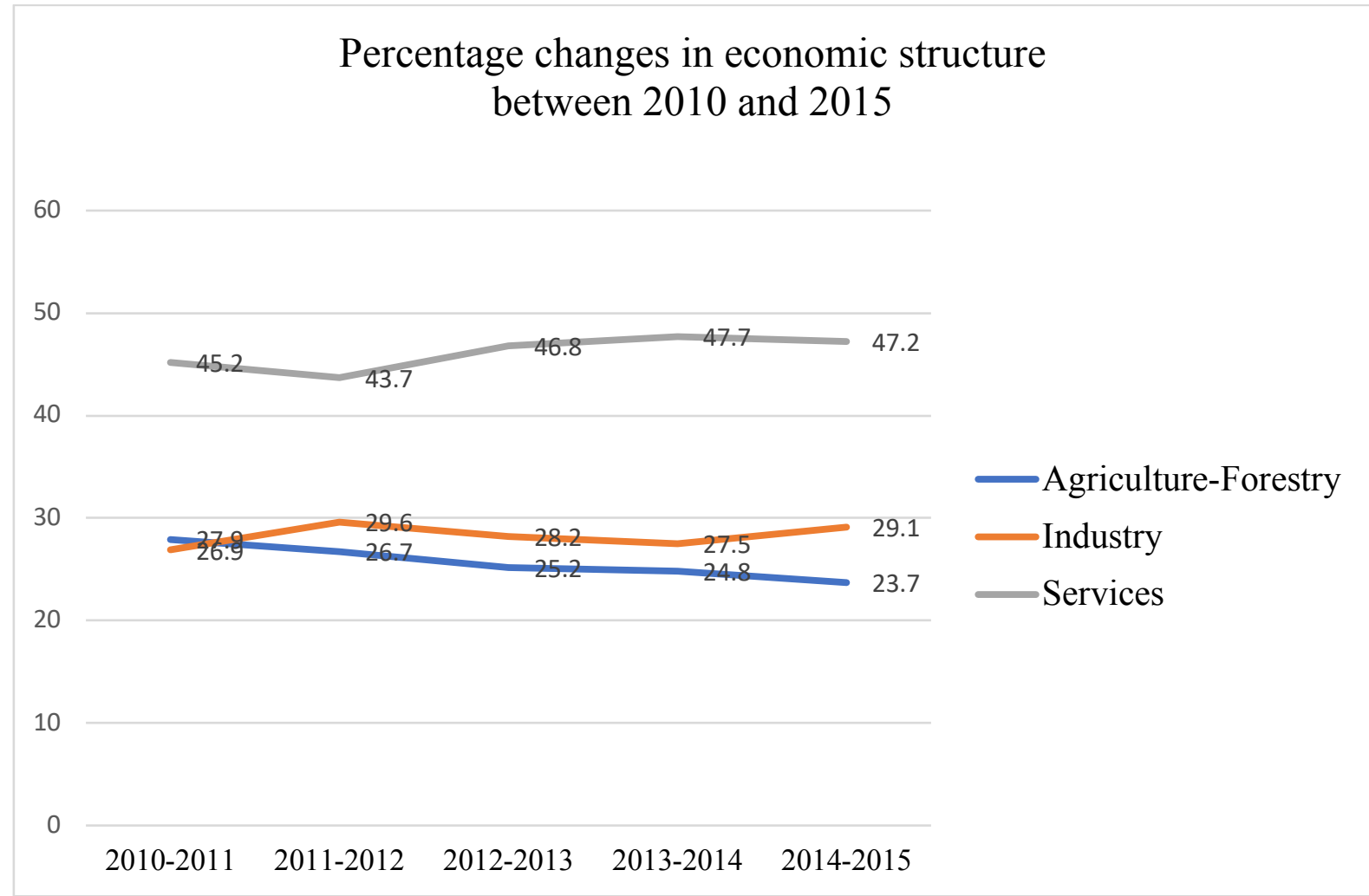

Source: adapted from the Ministry of Planning and Investment [MPI] (2016, p. 5).

Despite being the smallest sector in the economic structure, the agricultural sector holds the majority of the country's labour force (see Figure 4.4). The labour force in the sector has been declining, whereas the labour forces of the manufacturing and services sectors have increased. However, the changes at all these sectors happened only slowly, and the gaps between the agricultural sector and the other two remain wide. 
Figure 4.4. The trends of employment in the three sectors from 2010 to 2015

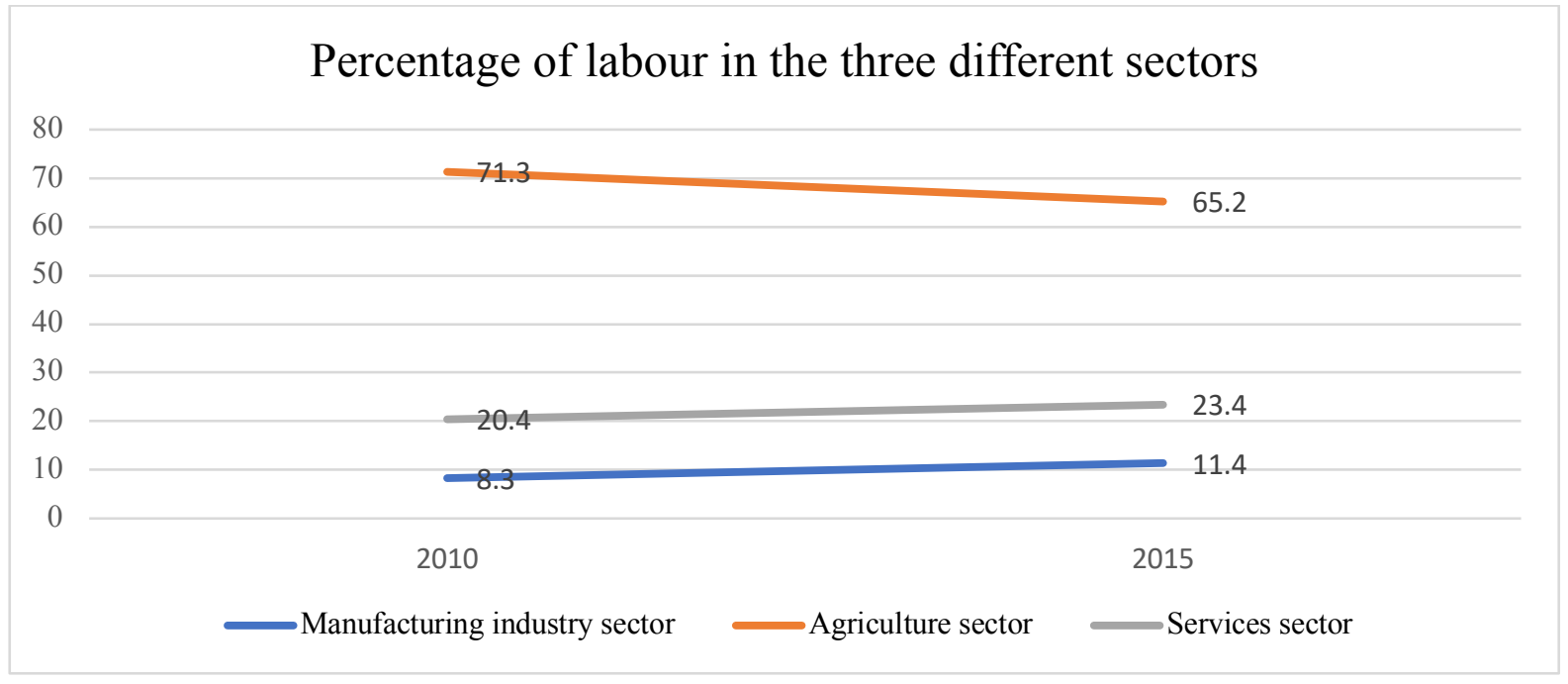

Source: adapted from MPI (2016, p. 11).

The World Bank (2014, as cited in United Nations, 2015) reported that the reliance on natural resources of the Lao economy is incapable of increasing employment. The need for labour in the natural resources sector was limited to only 22,000 people in 2013 . It was also found that more than a third of working people are unable to help themselves out of poverty with their current income. In addition, vulnerable employment remains common, particularly in agriculture, fisheries, and elementary work. However, the United Nations (2015) contends that the unemployment rate remains low due to the fact that the majority of rural people tend to work in spite of poor working conditions and low payment rates. Therefore, the United Nations (2015) suggested that the Lao economy needs to be diversified in order to enable decent job creation and inclusive growth in which women can have more and better employment opportunities.

Another challenge is that although Lao PDR has met the target of MDGs by halving its poverty rate and improving the lives of the poorest, the country is now facing increasing inequalities. Development remains centred in urban areas where infrastructure is more developed (MPI, 2016). Income and consumption inequalities between the rich and the poor, and between rural and urban people, are increasing. These trends have been studied and it has been found that "education, agricultural produce prices, insecure livelihoods, geographic location" (United Nations, 2015, p. 23), a weak manufacturing industry, and inadequate support for SMEs are the key determinants of the development gaps and ruralurban migration for work (United Nations, 2015; MPI, 2016). 
The World Bank (2018) stated that despite significant achievements in poverty and hunger reduction, and education and health improvement, the country remains challenged by child undernutrition in which the growth of 44 percent of children under five is stunted. Family planning remains strongly needed in some areas even though the national fertility rate is in decline. Maternal mortality and a lack of experienced birth helpers remain common. Therefore, the World Bank recommended that gender equality needs more focus, and to be integrated into the heart of Laos' national development plans. It is believed that this can be done successfully as the country has localised the Sustainable Development Goals (SDGs) into its national development plan.

In terms of gender equality promotion, of which the LWU is in charge, Laos has made progress in increasing women's participation in important positions. According to the report of the implementation of the seventh NSEDP, the LWU has been expanded to reach “930,963 members, 10,636 LWU groups and 1,550 local-level establishments" (MPI, 2016, p. 40). A number of female managers and leaders among these women from central to local levels have received political and technical training. The number of women occupying important positions and seats in the public sector has reached a certain percentage (see Figure 4.5).

Figure 4.5. Percentage of females in government organisations

\section{Gender proportions in government organisations}

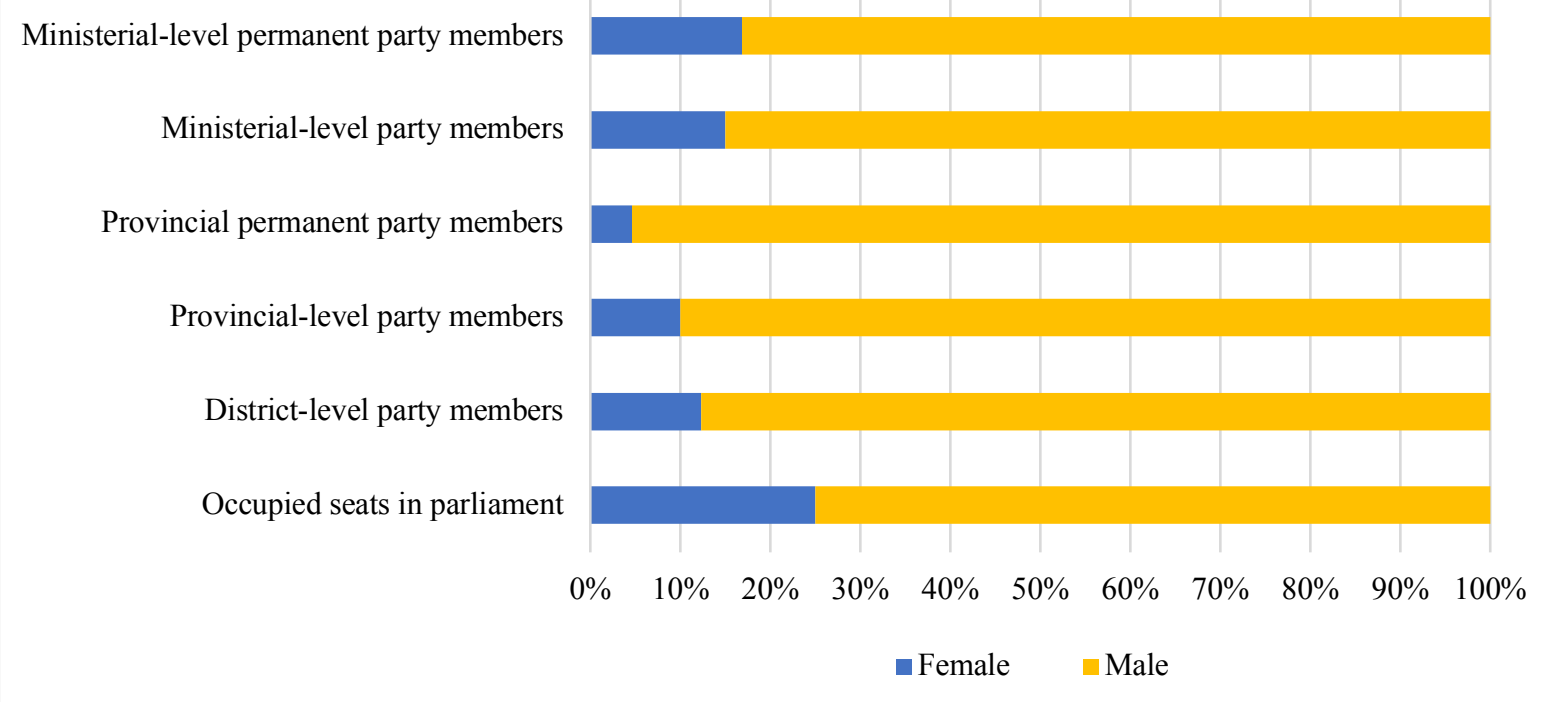

Source: adapted from MPI (2016, p. 40).

In addition, despite recent emphasis on girls' education, the gap between boys and girls at school remains. Gendered literacy disparities remain more common in rural and remote 
areas, where there is no road access, and where the majority of residents are ethnic people (MPI, 2016). Statistics show that the literacy gap between boys and girls aged between 15 and 19 is narrower than that of those aged between 20 and 24, possibly due to current increasing rate of girls at school, or due to boys undertaking more tertiary education. Table 4.2 below helps to illustrate more about other aspects of gender inequality in Laos.

Table 4.2. Gender equality and advancement of women indicators

\begin{tabular}{|c|c|c|c|c|c|}
\hline Indicator & $\begin{array}{c}2009- \\
10\end{array}$ & $\begin{array}{c}2010- \\
11\end{array}$ & $2011-12$ & $2012-13$ & $\begin{array}{c}2014- \\
15 \\
\text { Target }\end{array}$ \\
\hline $\begin{array}{l}\text { Ratio of girls to boys } \\
\text { enrolled in primary } \\
\text { school }\end{array}$ & 0.94 & 0.94 & 0.95 & 0.95 & 1 \\
\hline $\begin{array}{l}\text { Ratio of girls to boys } \\
\text { enrolled in lower } \\
\text { secondary school }\end{array}$ & 0.86 & 0.87 & 0.89 & 0.91 & 1 \\
\hline $\begin{array}{l}\text { Ration of girls to boys } \\
\text { enrolled in upper } \\
\text { secondary school }\end{array}$ & 0.82 & 0.81 & 0.83 & 0.84 & 1 \\
\hline $\begin{array}{l}\text { Ratio of girls to boys } \\
\text { enrolled in higher } \\
\text { education }\end{array}$ & & & & $\begin{array}{c}0.06 \\
\text { (vocational } \\
\text { level) }\end{array}$ & \\
\hline $\begin{array}{l}\text { Share of women in } \\
\text { wage employment in } \\
\text { the non-agricultural } \\
\text { sectors }\end{array}$ & & & $\begin{array}{c}34 \\
\text { percent }\end{array}$ & & \\
\hline $\begin{array}{l}\text { Proportion of seats } \\
\text { held by women in the } \\
\text { National Assembly }\end{array}$ & & & & 25 percent & \\
\hline
\end{tabular}

Source: MPI (2016, p. 50).

From the information about gender inequality in several aspects of Lao society, it can be seen that women have not had equal opportunities with men. The ratio of girls to boys at primary school begins to show better gender equality, but this ratio becomes gradually lower at higher education and tertiary levels. Women continue to form a much smaller proportion in wage employment in the non-agricultural sector, and constitute the lowest percentage of government officials in the National Assembly. Therefore, the government needs to keep pursuing gender equality in its sequenced national development plans.

\subsection{National policies on gender equality and international cooperation}

Following the achievements of the implementation of the seventh NSEDP, the eighth NSEDP (2016-2020) is in place to continue focusing particularly on poverty reduction, 
graduation from LDC status, and sustainable and inclusive growth (United Nations, 2015). Gender equality remains one of the development targets of the eighth NSEDP (MPI, 2016, p. 93). It sets out a general direction for women's development as follows:

Encourage women to access education to improve their professional/vocational/labour skills and ensure access to public health services, and increase their political awareness to enable them to contribute to socio-economic development and conservation and inheritance of the national culture; strengthen the Lao Women's Union at each level to be representative and protect women's and children's rights and interests; ensure equal rights and development between men and women in the areas of politics, economics, sociocultural affairs and the family; reduce discrimination and violence against women and children while promoting gender equality (MPI, 2016, p. 153).

To ensure the improvement of gender equality, specific targets have been set in the eighth NSEDP as set out in Table 4.3 below.

Table 4.3. Gender equality targets for 2016-2020

\begin{tabular}{|l|c|c|}
\hline \multicolumn{1}{|c|}{ Targets } & Increase & Decrease \\
\hline $\begin{array}{l}\text { Women in leadership and decision-making } \\
\text { positions in the Party, government and mass } \\
\text { organisations }\end{array}$ & $\geq 20 \%$ & \\
\hline Female members of the 8th National Assembly & $30 \%$ & \\
\hline $\begin{array}{l}\text { Female officials who have received training on } \\
\text { political studies, law, and public administration } \\
\text { and management }\end{array}$ & $\begin{array}{l}30 \% \text { of all } \\
\text { trained officials }\end{array}$ & \\
\hline $\begin{array}{l}\text { Implementation of recommendations of the } \\
\text { Committee on the Elimination of } \\
\text { Discrimination against Women (CEDAW). }\end{array}$ & $50 \%$ & \\
\hline $\begin{array}{l}\text { Implementation of the national action plan for } \\
\text { prevention and elimination of violence against } \\
\text { women and children by 2020. }\end{array}$ & $70 \%$ & $90 \%$ \\
\hline $\begin{array}{l}\text { Fertility rate of young women between 15 and } 19 \\
\text { years old }\end{array}$ & $50 \%$ & \\
\hline $\begin{array}{l}\text { Females entitled to land use rights, especially in } \\
\text { the cultivation and animal husbandry sector }\end{array}$ & $50 \%$ & \\
\hline Females in the service sector to 50 percent & & \\
\hline $\begin{array}{l}\text { The proportion of National Commission for the } \\
\text { Advancement of Women (NCAW) organisations } \\
\text { at all levels with improved functional structure }\end{array}$ & & \\
\hline
\end{tabular}




\begin{tabular}{|l|l|l|}
\hline $\begin{array}{l}\text { The proportion of supporting organisations with } \\
\text { improved functional structure }\end{array}$ & $80 \%$ & \\
\hline $\begin{array}{l}\text { Ensure gender equality in economic } \\
\text { opportunities, including access to resources such } \\
\text { as land, funds, technologies and basic }\end{array}$ & & \\
infrastructure (electricity, roads, markets). & & \\
\hline $\begin{array}{l}\text { Reduce discrimination and violence against } \\
\text { women and children }\end{array}$ & & \\
\hline
\end{tabular}

Source: adapted from MPI (2016, p. 155).

To meet these targets, some overall policies have been suggested in the eighth NSEDP. There appear to be three different classes of policies for gender equality. The first, possibly regarded as the most important intervention, is about deepening people's understanding of the Party's and government's policy and direction. This policy to some extent seems vague but the Party considers it compulsory for the purpose of socialist construction. The second class of policy is concerned with strengthening the capacity of relevant national organisations such as the National Commission for the Advancement of Women (NCAW) and LWU. These organisations need to be capable of raising people's, both men's and women's, awareness of policies and gender issues which are of concern, as well as of carrying out programmes in local areas to increase gender equality. The third policy category is about promoting regional and international cooperation in order to receive support from foreign governments and development partners (MPI, 2016).

To better understand the country's effort towards gender equality, it is necessary to further explore the LWU, given that this is a ministry-equivalent organisation on gender equality in Laos.

\section{Lao Women's Union:}

Lao Women's Union is a women's mass organisation, and the new name of the Lao Patriotic Women's Association, which was formed in 1955 under the Party for its revolutionary purpose. This organisation has reached many women at every level from local to central. Thousands of women across the country are members of the LWU (Ireson-Doolittle \& Moreno-Black, 2004). As a state pursuing socialism, the LPRP placed a number of strategic duties on this women's organisation. With the Party's direction, the first congress of the union in 1984 began to embark on encouraging all Lao women to realise women's "Three Goods" and "Two Duties". The "Three Goods" were initially to be a "good citizen, good mother, and good wife" (Ireson-Doolittle \& Moreno- 
Black, 2004, p. 27). However, these have been changed to "good citizen, good development, and good family", while the "Two Duties" remain the same: "national defense and national construction" (Lao Women's Union [LWU], 2015, p. 26).

At the seventh National Women's Congress in 2015, the State President, Choummaly Sayyasone, mentioned that the realisation of the LWU's slogan- "Gender equality improvement to be part of national development" - and the "Three Goods" need to be integrated into the implementation of the "Three Builds": "Building province as a strategic unit, building district as a strong comprehensive unit, and building village as a development unit" (MPI, 2016, p. 67; LWU, 2015). This indicates that Laos is placing an importance on villages as grassroots development agents but that this must happen under the supervision and direction of the central authority. Women are assigned with strategic tasks in order to encourage and include them in the development process. They are part of the implementation of the national development plans. Therefore, if we refer to the "Women in Development" (WID) approach, it can be seen that "these efforts to integrate women into development plans are generally known as part of the WID approach" (Koczberski, 1998, p. 395; JICA, 2006). This approach assumes that by incorporating women in development plans, their lives will be improved. Although the WID approach has made women's role in development known and raised gender awareness in society, women remain disadvantaged by the assumption of the approach that integrating women into development processes will automatically benefit women. This is due to the fact that this assumption prevents women's historical and sociocultural context from being taken into account (Koczberski, 1998). Koczberski (1998) also argues that the WID approach is part of modernisation theory in which women's traditional roles are ignored given that development is a linear process. In the case of Laos, women are expected to fulfil both development tasks and what is called the "good woman" norm. At the seventh National Women's Congress, a segment of the State President's speech is quoted as follows:

“..... it is a pride that Lao women maintain traditional characteristics such as being diligent, economical, industrious in the farm and textile work, devoted for children in the form of property inheritance, caring for children and husband, patriotic, ......all of which are contributions to national cultural preservation...." (LWU, 2015, p. 71).

It can be seen that women's roles in society and development are enormous, and society has a range of expectations towards women. The LWU is acting under the direction of 
the Party in order to ensure women fulfil their strategic tasks and duties for the purpose of socialist construction while women's traditional roles are also to be maintained.

In terms of international cooperation, Laos has maintained and increased its cooperation with regional and international development partners. Particularly, there is strong cooperation between the LWU and the women's organisations of other countries, including those in the Association of Southeast Asian Nations (ASEAN). The Vientiane Declaration on "gender promotion and ASEAN women partnership" has been adopted by the association members. The adoption of the declaration is a foundation for Lao women to improve their status in the international arena (MPI, 2016). Among many other countries, Laos has maintained its relationship with Germany in several respects, of which the Village Bank project targeted in this study is a distinguished one, as explored in more detail as follows.

\subsection{Village Bank structure and performance}

This section is focused on exploring the mechanism of Village Banks under the AFP project. Its management system, gender inclusion framework, and some performance data are included and consulted in this section. This exporation enriches the background information on the target area and people, and enables a better understanding of the project's impact on target women, and a proper justification of the findings.

\subsubsection{Village Bank system and administration}

The AFP project on Village Banks (VBs) was launched in 2009 with cooperation between a German development agency known as Deutsche Gesellschaft für Internationale Zusammenarbeit, Gmbh (GIZ), and the Lao government represented by Bank of Laos. The project is currently operating in six provinces and twenty-one districts (GIZ, 2015). The project was designed to help those who have no access to finance, given that these people constitute up to 64 percent of the adult population of Laos, with a higher proportion in rural areas (GIZ, 2018). The overall goal of the project is to ensure poor households have better access to effective financial services. To achieve this goal, the project is focused on three objectives. The first objective is building the management capacity of the Bank of the Lao PDR. This is to improve the Bank's regulation, governance and supervision of the microfinance sector. The second objective is building sustainable Village Bank Associations. The establishment of the associations is to ensure operational sustainability of VBs, particularly after the conclusion of the project. The 
third objective is strengthening Village Bank (VB) networks by improving services of VBs so that they are sustainable, satisfactory and appropriate for VB members (GIZ, 2018). Therefore, VBs under the AFP project are founded and operate under systematic management with the Bank of the Lao PDR as the ultimate supervisor.

Figure 4.6. The system of the AFP project

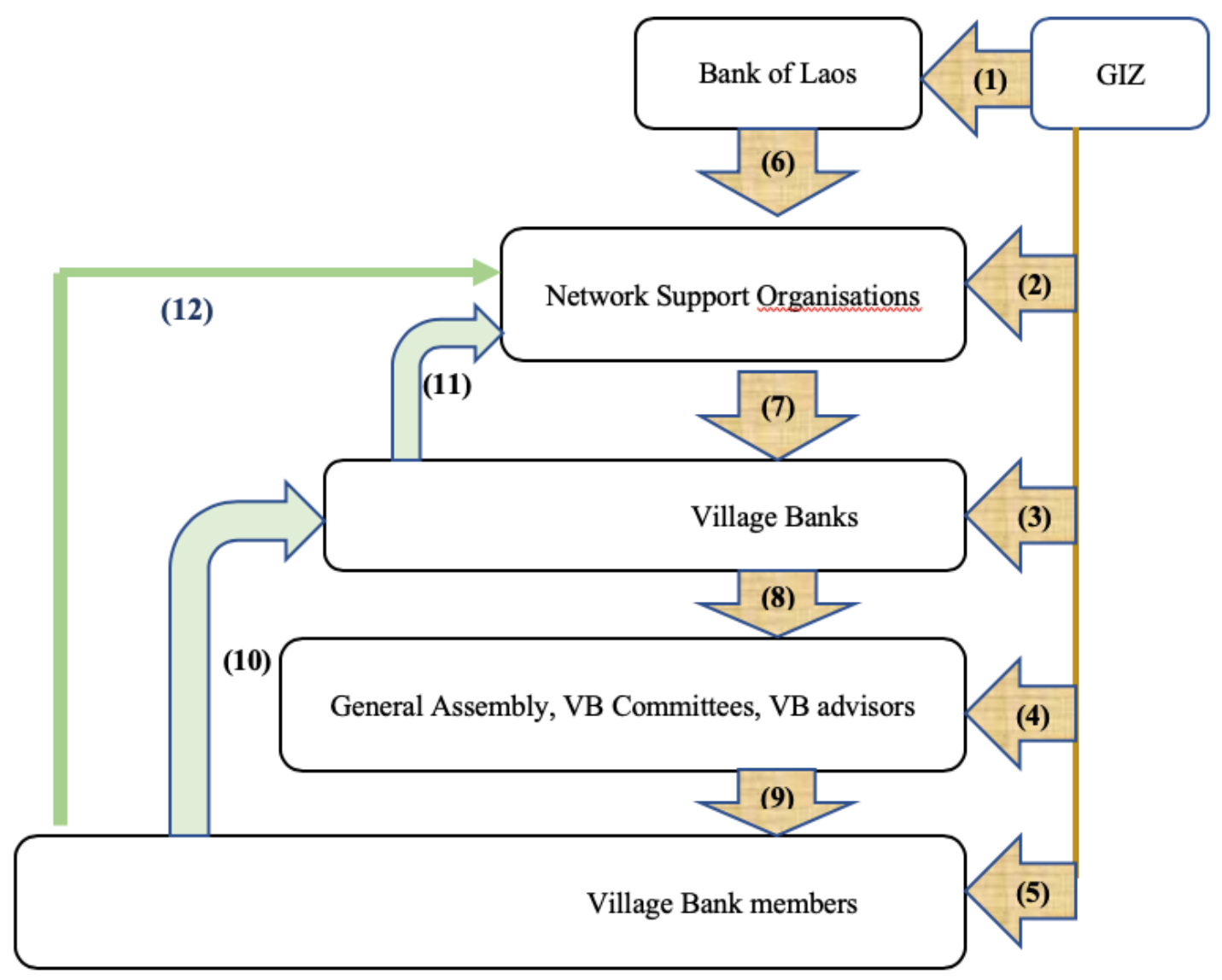

Source: Author, illustrated based on the report by Prochaska, et al. (2012).

(1) GIZ works with Bank of Laos at the macro level of providing policy advice to the Bank to improve its regulation, governance, and supervision of the microfinance sector. The AFP project is co-supervised by both organisations but GIZ has been improving the management capacity of BoL to ensure the sustainability of VBs after the project ends (Prochaska, et al., 2012).

(2) Network Support Organisations (NSOs) are founded as the second layer of administration of VBs. Each NSO is responsible for about 40 to $100 \mathrm{VBs}$. Their role is to set up and supervise VBs, represent and coordinate with VBs, and to provide outstanding financial services for VBs. These organisations are where VBs can deposit their surplus funds, or acquire wholesale loans in case of insufficient internal capital for VB members. 
These organisations maintain their existence upon fee payments and loan interest income from VBs (GIZ, 2014).

(3) VBs are established with the recognition of provincial and district authorities which render VBs a semi-formal status. VBs are owned by all members; and all villagers, regardless of age, gender, and ethnic origin, are encouraged to join the establishment. However, their membership is voluntary. Each VB consists of 75 members on average (GIZ, 2014). The VB structure is comprised of the Member General Assembly, the Village Bank Committee, VB Advisors, and VB members (GIZ, 2014).

(4) The General Assembly is formed with all the VB members, and plays the governing role in the VB. Almost all decisions in the VB are made by the Assembly by simply having at least 50 percent of the VB members' voice. It also elects the Village Bank Committee (VBC), and has the right to confirm or reject the appointment of VB advisors (GIZ, 2014). The VBC is responsible for overall administration, mainly for making decisions in regard to savings and credit operations of the VB. The number of VBC members ranges from four to six according to the VB's size and workload. Positions in the VBC basically include VB President, Vice President, cashier, main accountant, savings accountant, and credit accountant (GIZ, 2014). In addition, each VB has at least two VB advisors who are normally respected people from the village administration. Thus, these people are selected by the village administration to provide advice to the VBC in solving problems. Transparency and efficiency of the VB are ensured by its VB bylaws (GIZ, 2014).

(5) VB members receive financial training and education on investment besides their depositing and borrowing activities. These members hold either individual or joint accounts at the $\mathrm{VB}$, but joint accounts are found to be the most common option among VB members (GIZ, 2014).

(6) With the cooperation of BoL, all NSOs received their registration as Non-Deposit Taking Microfinance Institutions (NDTMFIs). These organisations operate under the supervision of, and receive training and recommendations from, $\mathrm{BoL}$ and GIZ. NSOs are trained and maintained for the sustainability of VBs, and are believed to be financially self-sustaining for the future depending on the growth of VBs. Up to 2012, there was only one NSO in Hongsa of Sayaboury province which appeared to be achieving financial selfsustainability (Prochaska, et al., 2012). 
(7) NSOs are in charge of two main tasks in assisting VBs. The first main task is technical assistance regarding financial education, book keeping, and procedural guidance. The second main task is as a financial intermediary by which NSOs provide wholesale loans for those VBs which do not have enough funds for their members (GIZ, 2014).

(8) The VBC members receive direct training, particularly on management and financial literacy, from their NSO. The VB pays its committee members with 15 percent of the annual net surplus of the VB. It is also noted that the VBC members are given a chance to work in the VB's NSO if she or he has the capacity (GIZ, 2014).

(9) Loans for emergencies, consumption, production, and trade activities requested by the VB members are decided on by the VBC. After a year, the surplus of incomes which is mainly generated through loan interest payments of up to three percent per month, is saved in the VB and paid back to VB members (GIZ, 2014).

(10) VB members must deposit money to their VB account with a minimum of normally $10,000 \mathrm{Kip}^{3}$ per month. The compulsory savings are applied to get VB members familiar with a saving habit after earning and before spending (GIZ, 2014).

(11) Every VB has to pay a five percent service fee annually to the NSO based on its total portfolio outstanding. VBs also deposit their surplus funds at their NSOs in order to generate more income as well as to ensure efficiency in fund management (GIZ, 2014).

(12) For those VB members who need a big loan, which is beyond the capacity of their own VB, they can send an application to the NSO for the loan (GIZ, 2014).

\subsubsection{Gender inclusion framework}

The general purpose of establishing VBs is to provide the rural poor with access to financial services. Thus, the project does not exclusively target women but the general poor. However, the need to increase women's participation in VB activities and services is accentuated. Women are included in the project by coincidence and deliberately (Prochaska, et al., 2012). The former means the risk-mitigation policies of the project encourage women to hold joint accounts with their husbands. This share of responsibility not only helps reduce risks for the VB but also empowers women (Prochaska, et al., 2012). The latter means each VB must have a minimum of two female committee members among the total of four to six members. Based on a survey in 2014, an increase

\footnotetext{
${ }^{3} 10,000 \mathrm{Kip}$ is approximately equivalent to USD1.2
} 
in the number of females in VBC was proposed. This was in order to incentivise other VB female members to participate in VB activities and services. This finding is based on a common response among VB female members that women's role modelling in the VBC helps increase their confidence and ambitions (GIZ, 2014). This proposed idea of increasing the number of VBC female members seems viable, but, in my opinion, it can also be interpreted that women's empowerment can only be achieved with the absence of men. Nevertheless, the implementation of these gender inclusion policies has resulted in several changes among rural poor and ethnic women, as below.

\subsubsection{Village Bank performance on women's empowerment}

The intervention of the project has increased access to financial services and decisionmaking among rural women. With the establishment of more than five hundred Village Banks across six provinces of Laos, there have been more than 62,000 accounts opened, in which 54 percent of these accounts are solely held by women, opened with VBs (GIZ, 2017). More than half of the VB members are women, who have access to 83 percent of the accounts. Women constitute about half of the participants in financial literacy training. The project continues to make progress on women's empowerment (GIZ, 2017). Nearly all target villages have received financial literacy training, reaching more than 25,000 villagers, in which the number of women account for 53.51 percent (GIZ, 2018). In terms of decision-making, the number of women holding decision-making positions in VBs is increasing with more than one third of all leadership positions in VBs occupied by women (GIZ, 2017). This is illustrated in Figure 4.7 as follows. 
Figure 4.7. Percentages of women in VBC

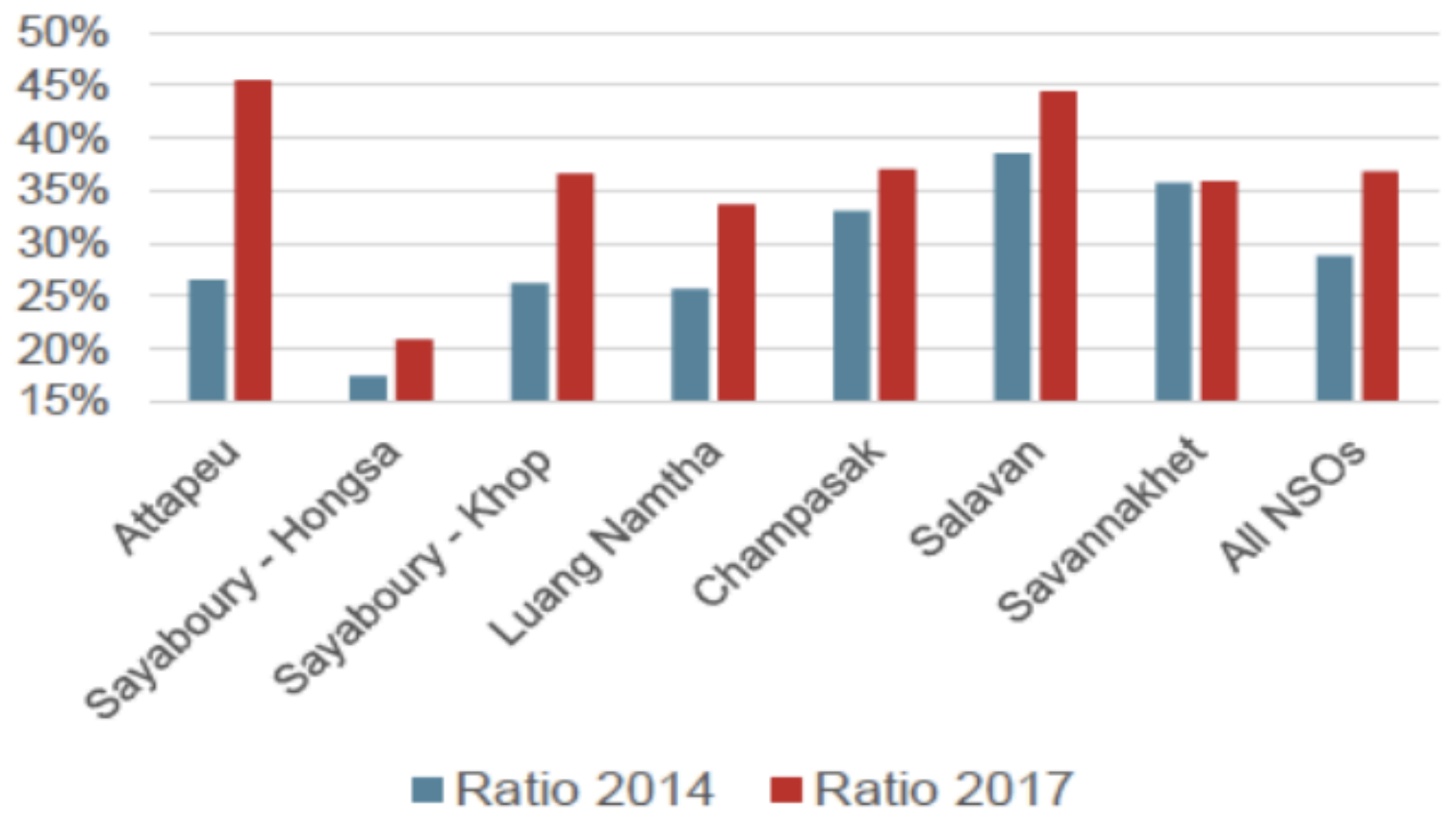

Source: GIZ (2018), slide 9.

These achievements of women's inclusion in the project have benefited women in several ways. The benefit most women mention is having access to financial services such as savings and credit. The dividend income on savings help increase household's spending, particularly on food and children's education. In addition, women see VBs as a place for communication and sharing ideas. It is where women acquire basic financial and technical skills. After years of participation, many women are now confident to speak out (Prochaska, et al., 2012). The perceived benefits of VBs for women are illustrated in Figure 4.8 as follows. 
Figure 4.8. Perceived benefits of VBs for women

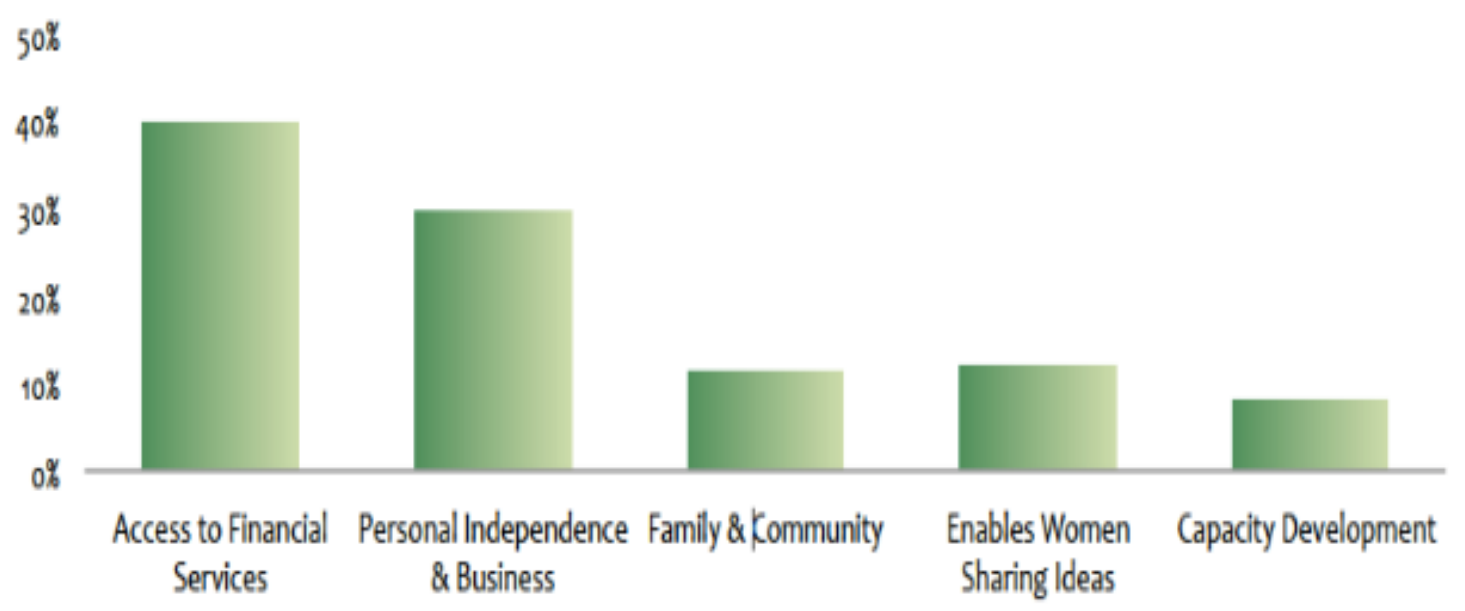

Source: Prochaska, et al. (2012, p. 30).

However, GIZ contends that women's empowerment continues to face challenges in Laos (Prochaska, et al., 2012). One reason is due to the commonness of male dominance in family and society. This means VB operations are dominated by men. Women's lack of education, particularly financial literacy, is known as the primary cause of male dominance in VB operations (Prochaska, et al., 2012). While joint accounts are considered as the best option for women's participation in VBs due to risk concerns, many women expressed the need to escape from holding joint accounts with their husbands to holding their own personal accounts. It is noted that joint accounts prevent women from having financial autonomy and from making decisions on personal businesses (Prochaska, et al., 2012). It can be seen that the women are facing basically two challenges, namely male dominance and lack of capacity.

\subsection{Three Village Banks in Luang Namtha Province—case studies}

This subsection provides specific information about the three VBs that were chosen to be case studies in this research. The data will draw a concise picture of what I go on to further build on in the findings and discussion chapters. It provides another foundation for better interpretation of my fieldwork data. 


\subsubsection{Donmoun Village Bank}

Ban Donmoun is a Khmu dominant village, located about ten kilometres to the south of Luang Namtha city centre, with a population of 1,021 people; 494 of whom are female (48.38 percent). The village has good access to roads. Donmoun Village Bank (DMVB) was established in September 2012 with a VBC of four members, two of whom are female. By July 2018, the number of accounts opened at DMVB reached 504. Women solely hold 142 accounts (28 percent) while the number of joint accounts is 208 (41 percent), and the number of accounts held solely by men is 154 (31 percent) (based on the data collected in July 2018 by the GIZ office in Luang Namtha province).

Table 4.4. Donmoun Village Bank data

\begin{tabular}{|c|c|c|c|c|}
\hline \multirow{2}{*}{ VBC members } & Male & Female & \multicolumn{2}{|c|}{ Total } \\
\hline & 2 & 2 & \multicolumn{2}{|c|}{4} \\
\hline \multirow[t]{2}{*}{ Members/Account holders } & Male & Female & $\begin{array}{c}\text { Joint } \\
\text { Accounts }\end{array}$ & Total \\
\hline & 154 & 142 & 208 & 504 \\
\hline Savings Balance & \multicolumn{4}{|c|}{ 2,603,745,000 Kip (about USD306,323) } \\
\hline Credit Balance & \multicolumn{4}{|c|}{ 2,411,944,000 Kip } \\
\hline Number of credits & \multicolumn{4}{|l|}{208} \\
\hline Overdue total & \multicolumn{4}{|c|}{$178,080,000 \mathrm{Kip}$} \\
\hline $\mathrm{PAR}^{4}$ & \multicolumn{4}{|l|}{$7.4 \%$} \\
\hline Interest income & \multicolumn{4}{|c|}{$160,971,500 \mathrm{Kip}$} \\
\hline Non-interest income & \multicolumn{4}{|l|}{ 5,989,000 Kip } \\
\hline \multirow{5}{*}{ Types of clients } & Emergency & 2 clients & \multicolumn{2}{|c|}{ 53,400,000 Kip } \\
\hline & Consumption & 15 clients & \multicolumn{2}{|c|}{$255,344,000 \mathrm{Kip}$} \\
\hline & Trading & 152 clients & \multicolumn{2}{|c|}{$1,186,750,000 \mathrm{Kip}$} \\
\hline & Production & 19 clients & \multicolumn{2}{|c|}{$112,200,000 \mathrm{Kip}$} \\
\hline & Others & 72 clients & \multicolumn{2}{|c|}{$60,000,000 \mathrm{Kip}$} \\
\hline
\end{tabular}

\footnotetext{
${ }^{4}$ PAR stands for portfolio at risk: a percentage rate of the total loans that are currently outstanding or unpaid.
} 


\begin{tabular}{|c|l|}
\hline Total income & $167,633,500$ Kip \\
\hline Total expenses & $118,993,000$ Kip \\
\hline Net earning & $48,640,500$ Kip \\
\hline
\end{tabular}

Source: adapted from data collected in July 2018 by GIZ in Luang Namtha province.

\subsubsection{Viengnuea Village Bank}

Ban Viengnuea is a Tai-Yuan dominant village, located about six kilometres to the south of Luang Namtha city centre, with a population of 1,424 people; 787 of whom are female (55.27 percent). The village has good access to roads. Viengnuea Village Bank (VNVB) was established in April 2014 with a VBC of five members, three of whom are female. By July 2018, the number of accounts opened at the VB reached 741. Women solely hold 230 accounts ( 31 percent), while the number of joint accounts is 351 (47 percent), and the number of accounts held solely by men is 160 (22 percent) (based on the data collected in July 2018 by the GIZ office in Luang Namtha province).

Table 4.5. Viengnuea Village Bank data

\begin{tabular}{|c|c|c|c|c|}
\hline \multirow{2}{*}{ VBC members } & Male & Female & \multicolumn{2}{|c|}{ Total } \\
\hline & 2 & 3 & \multicolumn{2}{|c|}{5} \\
\hline \multirow{2}{*}{ Members/Account holders } & Male & Female & $\begin{array}{c}\text { Joint } \\
\text { Accounts }\end{array}$ & Total \\
\hline & 160 & 230 & 351 & 741 \\
\hline Savings Balance & \multicolumn{4}{|c|}{ 5,510,398,000 Kip (about USD648,282) } \\
\hline Credit Balance & \multicolumn{4}{|c|}{ 5,361,986,000 Kip } \\
\hline Number of credits & \multicolumn{4}{|l|}{242} \\
\hline Overdue total & \multicolumn{4}{|c|}{$807,600,000 \mathrm{Kip}$} \\
\hline PAR & \multicolumn{4}{|l|}{$14.1 \%$} \\
\hline Interest income & \multicolumn{4}{|c|}{$209,644,000 \mathrm{Kip}$} \\
\hline Non-interest income & \multicolumn{4}{|l|}{ 4,066,000 Kip } \\
\hline & Emergency & 5 clients & \multicolumn{2}{|c|}{ 28,400,000 Kip } \\
\hline Types of clients & Consumption & 272 clients & \multicolumn{2}{|c|}{ 5,231,846,000 Kip } \\
\hline
\end{tabular}




\begin{tabular}{|c|l|c|c|}
\hline & Trading & 8 clients & $101,740,000$ Kip \\
\cline { 2 - 4 } & Production & 0 & 0 \\
\cline { 2 - 4 } & Others & 0 & 0 \\
\hline Total income & $217,074,000 \mathrm{Kip}$ \\
\hline Total expenses & $18,550,000 \mathrm{Kip}$ \\
\hline Net earning & $198,524,000 \mathrm{Kip}$ \\
\hline
\end{tabular}

Source: adapted from data collected in July 2018 by GIZ in Luang Namtha province.

\subsubsection{Namdaeng Tai Village Bank}

Ban Namdaeng Tai is a Hmong dorminant village, located about 25 kilometres to the east of Luang Namtha city centre, with a population of 1,298 people; 605 of whom are female (46.61 percent). This village has good access to roads. Namdaeng Tai Village Bank (NDTVB) was established in June 2015 with a VBC of five members, two of whom are female. By July 2018, the number of accounts opened at NDTVB reached 132. Women solely hold 29 accounts (22 percent), while the number of joint accounts is 61 (46 percent), and the number of accounts held solely by men is 42 (32 percent) (based on the data collected in July 2018 by the GIZ office in Luang Namtha province).

Table 4.6. Namdaeng Tai Village Bank data

\begin{tabular}{|c|c|c|c|c|}
\hline \multirow{2}{*}{ VBC members } & Male & Female & \multicolumn{2}{|c|}{ Total } \\
\cline { 2 - 5 } & 3 & 2 & \multicolumn{2}{|c|}{5} \\
\hline \multirow{2}{*}{ Members/Account holders } & Male & Female & Joint \\
Accounts & Total \\
\cline { 2 - 5 } & \multicolumn{2}{|c|}{42} & 29 & 132 \\
\hline Savings Balance & $332,657,000$ Kip (about USD39,136) \\
\hline Credit Balance & $224,197,000$ Kip \\
\hline Number of credits & 27 & \\
\hline Overdue total & $13,000,000$ Kip \\
\hline PAR & $0.0 \%$ \\
\hline Interest income & $8,443,000$ Kip \\
\hline
\end{tabular}




\begin{tabular}{|c|l|c|c|}
\hline Non-interest income & $2,152,000$ Kip \\
\hline \multirow{4}{*}{ Types of clients } & Emergency & 3 clients & $16,000,000$ Kip \\
\cline { 2 - 4 } & Consumption & 0 & 0 \\
\cline { 2 - 4 } & Trading & 29 clients & $158,197,000$ Kip \\
\cline { 2 - 4 } & Production & 0 & 0 \\
\cline { 2 - 4 } & Others & 0 & 0 \\
\hline Total income & $10,595,000$ Kip & \\
\hline Total expenses & $2,844,000$ Kip \\
\hline Net earning & $7,751,000$ Kip \\
\hline
\end{tabular}

Source: adapted from data collected in July 2018 by GIZ in Luang Namtha province

By bringing data of the three villages together, we can see the similarities and differences between the three VBs as shown in Figure 4.9. The comparisons and contrasts of the data provide us with some fundamental insights of the three VBs. This is essential and complementary to the interpretation of the research findings.

Figure 4. 9. Members of the three Village Banks

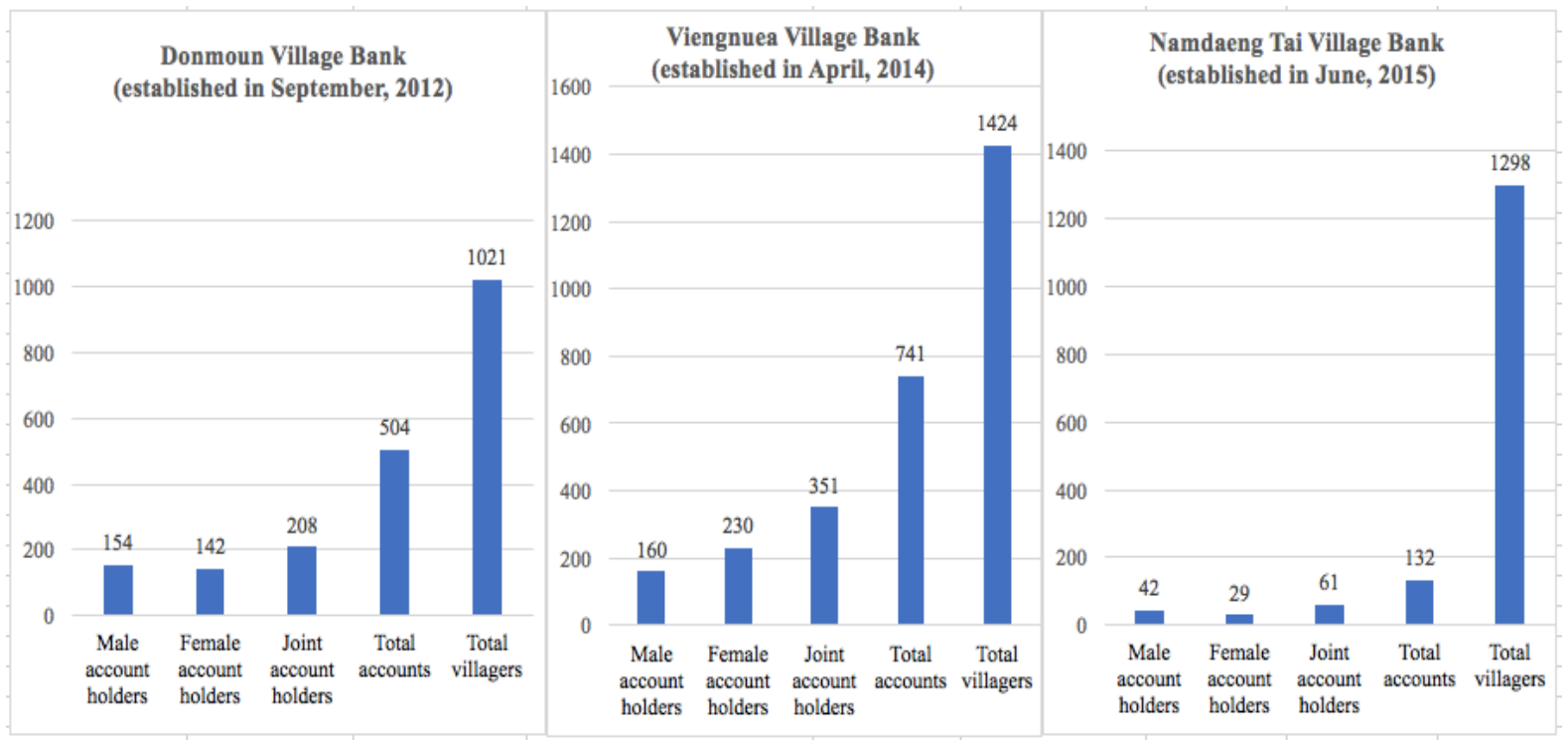

Source: adapted from data collected in July 2018 by GIZ in Luang Namtha province.

DMVB and VNVB share a similarity in the proportion of villagers having an account at VBs. About half of residents in both villages have an account at their VB. In contrast, only ten per cent of villagers in Namdaeng Tai village hold an account at their VB. Unlike 
VNVB, the number of accounts held by men at DMVB and NDTVB is greater than the number held by women. However, joint accounts remain the most common option across the three VBs. It is also noted that, although DMVB is the oldest among the three, VNVB, which was founded about two years later, has the highest growth rate. It seems reasonable for NDTVB to have the lowest growth rate as it is the youngest, but its progress is too much slower than the progress of the other two, given that it is only one year younger than VNVB. The following figure (Figure 4.10) further illustrates the growth of the three VBs.

Figure 4.10. Account balance at the three Village Banks

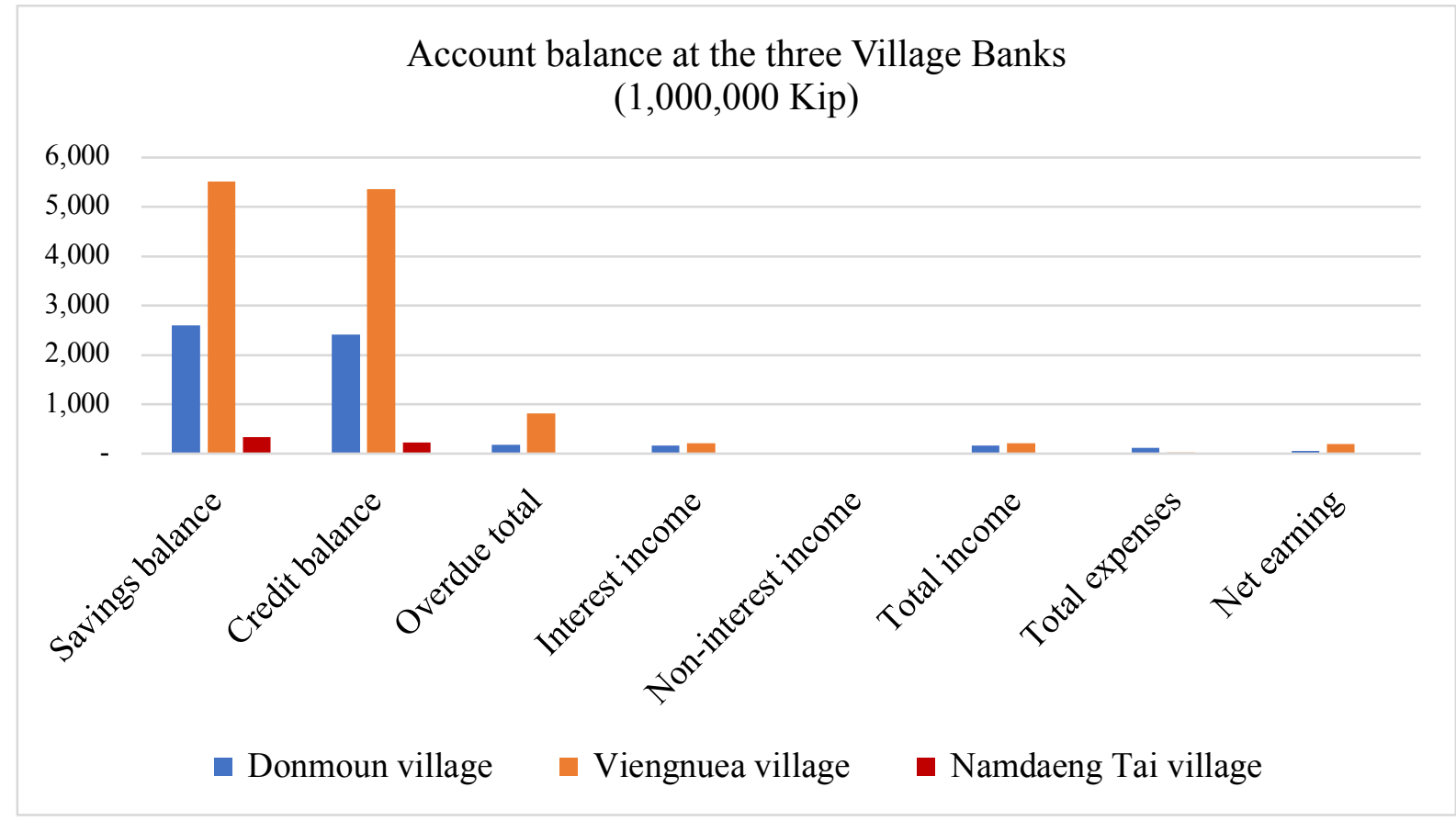

Source: adapted from data collected in July 2018 by GIZ in Luang Namtha province.

It is seen that VNVB is leading in most banking services and activities. Particularly, both the savings and credit balances of the bank have reached over five billion Kip ${ }^{5}$ while those of DMVB are nearly half those of VNVB. In contrast, NDTVB underperforms in most areas of banking services and activities with just a few hundred million Kip in either its savings or credit balance. It is interesting that the total expenses of DMVB are more than six times higher than those of VNVB, while its total income is smaller than that of VNVB. This indicates that the use of resources at DMVB is not as efficient as the use of resources at VNVB. This difference can be partly explained with the following figure (Figure 4.11).

\footnotetext{
${ }^{5}$ Five billion Kip is approximately equivalent to USD600,000.
} 
Figure 4.11. Types of loans taken by VB members

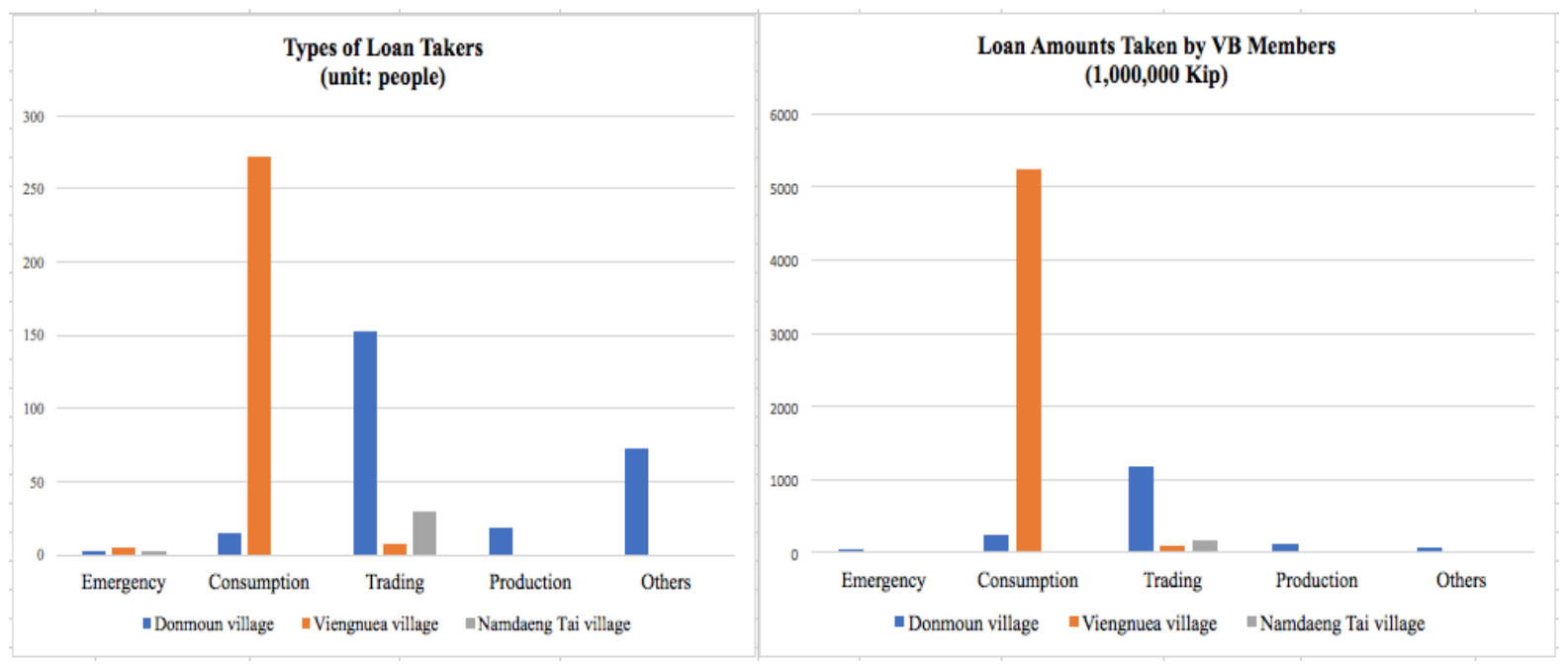

Source: adapted from data collected in July 2018 by GIZ in Luang Namtha province

It can be seen in Figure 4.11 that most VNVB members spend their loans on consumption while DMVB members tend to borrow money for trading. In other words, DMVB members tend to use the money from the VB to earn incomes, whereas VNVB members mainly use the money for non-income generating activities. This provides a few assumptions. The first assumption is that VNVB members do not rely on VB loans to earn income. The loans are paid back to the VB with other sources of income. In contrast, DMVB members rely on VB loans to enable their businesses and investment. The second assumption is that VNVB members have less confidence in investment than those of DMVB. To verify these assumptions, there is a need for further study.

In addition, it is interesting to note that NDTVB members have used only two types of loans, namely emergency and trading. Although the total of the trading loans taken at NDTVB is so much smaller than the total at DMVB, it is substantially larger than the total at VNVB. Thus, the total of the trading loans taken at VNVB is the smallest among the three VBs, despite its outperformance in other areas. These variations among the three ethnic groups are explained to some extent in this research. However, any particular investigation on these variations would be interesting and valuable.

\subsection{Feminist perspectives on Laos' initiatives on women's empowerment}

If the approach of the Lao government, particularly the Lao Women's Union, and the approach of the AFP project are taken into comparison and contrast, it can be seen that the approach of the AFP project shows more conformity to the GAD approach, the theoretical standpoint of this study. The policies of Laos tend to integrate women into the 
development process without much attention to the need to consider cultural backgrounds, while the AFP project does acknowledge that some of the local cultural and traditional beliefs are barriers to the success of VBs. The overall approach of the LWU is seen as a strategy to integrate women into the development process in order for women to be equal to men. This strategy is part of the WID approach, and could be criticised for ignoring gender relations and ideology (Drolet, 2010; Tinker, 1990; Visvanathan et al., 1997). Apparently, the AFP project is working at the grassroots level, and promoting women to help them meet their strategic needs by its gender inclusion framework. However, the project has not conducted any particular research on the influence of cultural beliefs on the achievement of the project. This would be because the project believes that cultural values, despite its acknowledgement, do not have much effect on women's participation in the project, given that only a small proportion of women attach their challenges to cultural norms (see Prochaska, et al., 2012).

\subsection{Summary}

In this chapter, the Lao social, economic, and political situations have been explored in detail in order to pave a foundation for the understanding about, and interpretation of the findings of this study. The backgrounds of the three ethnic groups have also been revealed. I have also shown how the national context has an effect on gender equality, particularly the lives of women. The chapter also involves a thorough investigation of national and some international intentions and initiatives on gender equality. It ends with a critical assessment of the three Village Banks, and a reflection on the country's and project's approaches towards gender equality in Laos. These data and this information will enable me to process the findings and present and discuss these with ease, confidence and validity in the following chapters. 


\section{Chapter 5: Presentation of findings}

\subsection{Introduction}

This chapter provides the main findings of this study. The findings are divided into three sections according to ethnicity. Each section presents the findings from a particular ethnic group in themes, with key direct quotes from the interviews and focus groups. The respective findings based on ethnic differences in this chapter will be integrated into the next chapter in order to enable discussion of their meanings and implications.

\subsection{Donmoun Village findings}

\subsubsection{Khmu women's perceptions of empowerment}

The focus group of Khmu women simply explained empowerment as being proud of themselves, and being helped by their husbands. To explain the former, the women stated that, besides the pride of economic wealth, a woman also needs to believe in herself that she is doing the right thing. The latter is explained in that, although their husbands are mainly in charge of hard work outside the house, they want their husbands to help them in domestic work, and help them pay household debts to the VB (Focus group, 7/7/2018). To illustrate the latter point, a story from a participant in the focus group is quoted as follows:

I think I am the most important person in my family because almost everything in the family is done by me, except a few hard duties done by my husband, but he still says that I am not good enough. He usually goes out drinking, and comes home late at night. I am both angry as a result of his ignorance of family, and worried for his health. I would not get angry if he did it during festival time only (Focus group, 7/7/2018).

In the Khmu community, the husband is always the most important due to his physical advantage by which he can do either hard or light work. Khmu women do not find this frustrating but they feel like they are being treated unfairly by their husbands with respect to their husbands' tobacco and alcohol additions. This addictive habit is believed among Khmu women to be unchangeable, so they rather remain silent and live with it. The women also elaborated further that what most concerned them was their husband's health, rather than his ignorance of family or nonperformance of housework. An interview participant put it that "I never get angry with my husband for not helping me with domestic chores, I am happy with it" (Interview, 6/7/2018). Another woman contends 
that men being higher than women is sometimes about paying respect, not gender inequality. She said, "We [women] respect men because they do hard work and lead the family" (Interview, 6/7/2018).

\subsubsection{VB benefits in respect of women's family obligations}

Women's VB membership has contributed to their fulfilment of family obligations. With the access to financial services that the VB provides, Khmu women now have a financial source to ensure their children's education. They can either deposit money at, or borrow money from, the VB. These services are needed for the community, given that they are struggling to make a living and support their children's and youngers' education. An interviewee revealed:

Rice from the farm is just enough for the family; there is no extra for sale or for relatives. Money for children's education is from the small plot of rubber trees (Interview, 6/7/2018).

Another interviewee told me that she has only about two hundred rubber trees so these take her and her husband about two hours each day to manage. This is her family's only source of income, and it is not enough to cover family expenses (Interview, 6/7/2018). Therefore, family burdens are partly lifted by their reliance on VB services. To further illustrate this point, a story of one interviewee is quoted:

I deposit money at VB for my children. I do my best to help one after the other. Although some of my children have been able to help themselves, there are some others who still need my help. If one falls into difficulties, I will use the money to help him or her up. If my children can find a job after graduation, I will be very happy (Interview, 6/7/2018).

Family burdens are borne not only by parents but also by older brothers and sisters. Those who are the eldest child in the household are likely to share the responsibility of supporting their younger siblings until they are financially strong. One VB committee female who is an eldest daughter-in-law expressed:

I am the eldest sister-in-law in the family, so I have to save money to support my brothers-in-law and sisters-in-law in their education. One is now studying in China, one is studying education in the country, and the others are studying accounting and politics (Interview, 6/7/2018).

This interview participant remarried to her current husband and has two children. Her previous marriage was a failure due to her ex-husband's addiction to drugs. She has a 
poor health so her current family obligations and the trauma from her previous marriage prevent her from daring to think about doing something new and good for herself. In referring to her trauma, family obligations, and poor health, she noted, "I will be able to do things as others do once my current family obligations are lifted" (Interview, 6/7/2018). Despite the challenges, she appears to be one of the most determined women in the community.

\subsubsection{Benefits for VBC members versus ordinary VB members}

VB benefits are more obvious for VBC members. This can be attributed to a number of reasons. The first reason is that all VBC members, including females, are elected based on their basic education level, particularly their financial literacy. The second reason is that all VBC members are selected through an election process by all VB members. The third reason is that VBC members have been exposed to training opportunities at the VB. These three main advantages seem to have distinguished VBC members from ordinary $\mathrm{VB}$ members, in terms of making use of the VB. In an interview with a VBC member, she said, "I have learned to use numbers, and have become confident with them after becoming a VBC member" (Interview, 6/7/2018). Similarly, another VBC female put it that "I am proud of my membership at the VB because I have learned many things, and sometimes I have the opportunity to do training, but I still lack confidence" (Interview, 6/7/2018). This interviewee is the woman I mentioned in the previous section who is the eldest sister-in-law who has to support her youngers-in-law financially in their education.

In contrast, ordinary VB members feel benefited when their saving balance increases, and when they are able to access money when they are in need of it. The increase in savings makes them happy and confident, and allows them to gain a sense of security. In an interview with an ordinary VB member, she said, "I feel a change in myself as my money deposited at the VB increases gradually" (Interview, 6/7/2018).

\subsubsection{The effect of the VB on women's mobility}

The establishment of the VB has resolved women's concerns about having to travel to the city to access the financial services of commercial banks. The village is only about ten kilometres from the city centre but women rarely travel there. A woman revealed that she has never travelled to other cities apart from the city centre of Luang Namtha since her birth due to poverty and family obligations (Interview, 6/7/2018). Because of poverty, many women tend to avoid using the financial services at commercial banks. Another interviewee said that there are two reasons that she does not use financial services at 
commercial banks. She said, "I would use the services at commercial banks if I had a lot of money. Another barrier is my lack of driving skills" (Interview, 6/7/2018). Some women complained that the borrowing process at commercial banks is complicated and that they are not capable of handling the process. In addition, they think that they would be judged as poor at commercial banks. Therefore, the VB is an ideal solution for the concerns of these women. In one participant's response, she said "I am happy with the VB even though it is a micro financial services unit" (Interview, 6/7/2018). However, it can be seen that the presence of the VB has reinforced women's existing lack of mobility.

\subsubsection{Challenges facing women}

Besides poverty, family obligations, men's ignorance of family, and their lack of driving skills, as mentioned previously in this chapter, Khmu women also face many other challenges. A woman, despite her membership of the VBC, remains unconfident in the community and in her position at the VB. She believes that it is due to her lack of high education (Interview, 6/7/2018). Another participant revealed that her health is the biggest barrier in her life. She is quoted as follows:

I believe that I would not be able to learn anything new due to my health problem. I may be able to learn technical things without too much mental concentration and energy. I understand that taking care of myself is the first thing to do because my health is fundamental for maintaining the wellbeing of my family (Interview, 6/7/2018).

In the focus group with Khmu women, they asserted that women's poor health compromises their ability to earn an income and to contribute to other provisions for the family so it brings about family poverty. They confessed that their poor health is a result of their unsafe eating habits. A participant in the focus group is quoted as below:

Now everything is bought from the market, particularly vegetables and noodles. Everything is contaminated with chemicals. There is no organic produce. Everything is grown with fertilisers. Before, we mainly relied on the forest but now people tend to rely on the market. It is our fault for not washing everything properly with salt and hot water before consuming. Laziness is blamed for all. We have plenty of land to grow vegetables but we don't want to grow them by ourselves. We like to buy them from the market. This is our own mistake (Focus group, 7/7/2018).

It is seen that the women completely blame themselves for their own health issues. Some others voiced that the reliance on the market is a result of their increasing engagement in 
the rubber tree industry which means they do not have time to grow vegetables on their own plots of land.

In addition, despite a common assertion among the Khmu women that there is no male resistance against women or traditional practices that impact negatively on women in the Khmu community, some Khmu women are not comfortable in talking to strangers in the presence of their husbands. When interviewing a woman whose husband joined the interview, I noticed that she was obviously unconfident and avoided answering several of my interview questions. She gave very short answers to most of my questions in a very low voice. This implies that Khmu women are not entirely free of male dominance.

\subsubsection{Women's strategies to overcome these challenges}

Most of the Khmu women in the study have no ideas about strategies to overcome their challenges. A common suggestion was working harder, as they believe that life will automatically be better with personal diligence. This includes being diligent in producing their own food rather than relying on the market, which reliance they believe is increasingly damaging their health. In regard to gender concerns, they asserted that there is no need to worry about men's status in the family, given that it is normal and acceptable in Khmu community.

\subsection{Viengnuea Village findings}

\subsubsection{Tai-Yuan women's perceptions of empowerment}

The Tai-Yuan women at the focus group suggested that empowerment means being proud of themselves. They explained "being proud" as a mental state where people feel content with what they have. These women perceive empowerment as the ability to control their mind in order to avoid being greedy or too ambitious about everything in life. To illustrate this, a statement from the focus group is quoted as follows:

Being empowered means being proud of myself for what I have, not to catch up with other people for what they have. It means accepting what my family has, not thinking beyond my ability and reality. Anything either achieved by me or my husband is for the family, there is no need to worry about it (Focus group, 4/7/2018).

Despite this perception, the Tai-Yuan women expressed their aspirations as follows:

$\checkmark$ having a family of harmony, cooperation, and happiness;

$\checkmark$ being healthy mentally and physically; 


\footnotetext{
having economic wealth;

$\checkmark$ having a chance to travel overseas;

$\checkmark$ being able to send their children overseas for education; and

$\checkmark$ having knowledge to improve their lives.
}

They revealed that if these aspirations were achieved, there would be changes in their lives. They would become good people; their lives would be more pleasant; they would be well known in society, have dignity, and a happy family. However, they contended that people around them have become richer and richer, so that there is a need to push themselves to do more in order to catch up with other people. Otherwise, their lives will be lagging behind others and filled with upset. A focus group participant said, “People's increasing economic richness makes me feel unsatisfied with my current economic wealth so I have to do more to catch up with them" (Focus group, 4/7/2018). The women asserted that the only thing they need to achieve their aspirations is money. Therefore, they would like to see job creation in the area, particularly in their village, in order to provide them with income earning opportunities.

\title{
5.3.2. The effect of the VB on villagers' security
}

Before the establishment of the VB, the villagers tended to borrow money from each other, particularly in cases of emergency. This had resulted in several conflicts between the borrowers and lenders, even among brothers, sisters, and close relatives. Now this kind of issue is no longer seen due to the presence of the VB. In the focus group with TaiYuan women, a participant is quoted as follows:

\begin{abstract}
Before the presence of the VB, people tended to borrow money from relatives so this resulted in many conflicts. Now those incidences are no longer seen in the village because people just need to come to the VB and borrow money. In cases of emergency, for example because someone is seriously sick, they are no longer at risk of death because of family financial shortage. Without the VB, many poor villagers wouldn't know what to do when money is seriously needed (Focus group, 4/7/2018).
\end{abstract}

Therefore, the focus group participants asserted that the biggest contribution of the VB to their lives, as well as to the lives of all the villagers, is the sense of security it creates. It is noted that relying on commercial banks for such emergency cases would be regrettable. A quote from the focus group is cited as below:

Before the VB, we were very worried about applying for a loan at commercial banks because it might take a month to get it approved. This 
worries us as it may cause losses, particularly loss of life before we get the money. Unlike commercial banks, the VB is very flexible (Focus group, 4/7/2018).

It can be seen that the VB has had a psychological impact on the villagers as well as the women rather than a physical effect. It appears that the women talked most about its emergency credit facility rather than any other benefits.

\subsubsection{The effect of the VB on VBC members}

Women's membership of the VBC increases their self-worth given that not everyone is able to gain that position. A female VBC member said that her membership is the result of a selection process by all the VB members who have trust in her. Therefore, holding the position means she has a significant role in community, as well as in her family, so that this increases her confidence (Interview, 3/7/2018). In the interview, she said:

I believe that people's views of me have changed since my VBC membership because I am now working in a banking-like area (Interview, 3/7/2018).

It is seen that, despite the small scale of the VB, women who are VBC members consider themselves to be working in an official bank. The offering of training and position advancement opportunities at the VB provides women with the hope of career advancement. According to GIZ (2014), those who make a good progress in the VB have a chance of being promoted to work in the NSO.

Another female VBC member was assertive on the topic of her role in working for the VB. She firmly expressed that the VB had provided the villagers with access to financial services so that this was an opportunity for her to contribute to the wellbeing of the villagers. In the interview, she said, "I am proud of my VBC membership, and I strongly believe in this bank system as it is based on a firm management system governed by the Bank of Laos" (Interview, 3/7/2018).

From the interviews with these two female VBC members, one possibility is seen here: that is, that strategic gender needs seem to be being met among VBC members alone. This would mean that achieving empowerment requires an increase in the number of female VBC members (GIZ, 2014), however, as noted earlier, this could also lead to the assumption that women can only be empowered with the absence of men. 


\subsubsection{The limited effect of the VB on ordinary VB members}

The impact of the VB tends to be restricted to the household level. Ordinary VB members tend to benefit from the VB in terms of meeting their practical needs rather than their strategic needs. In an interview with an ordinary VB member, she said:

My participation in the VB has no effect on how people view me. I think people's image of me remains the same as before. This is because I see no change in people's behaviour towards me. However, my family members, such as my husband and children, may view me differently than they did before. They may see me as a better person (Interview, 3/7/2018).

Another woman's explanation for her participation in the VB is very simple. With a humble voice and sitting position, she said:

I became a member of the VB in the second year of its establishment. I don't have much money to save in the bank. I deposit it in the VB because I want to earn some income at the end of the year, for no other reason.... [I] have little, deposit little. I choose the VB where I can deposit as little as I have. I don't go to use commercial banks because I am shy about how little money I have. I am worried that people will judge me for my poverty (Interview, 3/7/2018).

She revealed further that her VB membership has no effect on her thinking and feeling about herself. She also believes that people's image of her remains the same as before. However, she asserted that she is a very important person in her family. In talking about this, she said:

All my family members know my ability. I am the best in the family. I do almost everything, including cooking, preparing food for my husband to take to work, and for my children to take to school, and feeding the animals. As my husband works hard during the day, I have to make sure food is ready for him every day. Without me, the family would be unstable (Interview, 3/7/2018).

Unlike her, some other participants revealed that they also use the financial services of commercial banks. Therefore, it is seen that poverty tends to prevent poor women from approaching other sources of finance and opportunities. The VB is where they feel most comfortable but they benefit from it the least. The benefit is restricted to having access to financial services and a chance of making a small income. 


\subsubsection{Spiritual practice, technologies, and gender equality}

Tai-Yuan women revealed how spiritual belief is practised, and how it has an effect on gender equality. There is a difference between those ethnic groups who practise spiritual belief at home and those who practise it at the temple. In talking about this, a woman said:

We practise Buddhism so there is no particular spirit worshiped at home. We worship spirits mainly at the temple so it doesn't affect those who have a matrilocal or patrilocal culture. Both men and women from either group can join the spiritual practice equally without any distinction. But in some ethnic cultures such as Tai $\mathrm{Dam}^{6}$, they follow a patrilocal culture, and worship the spirits of their ancestors at home, so men are in charge of practising and maintaining the spiritual belief. Therefore, men can't move to live with their in-laws after marriage (Interview, 3/7/2018).

As a result of worshipping at the temple, the Tai-Yuan women asserted that they face no gender exclusion in the community. The interviewee added that "I see no barrier in my way towards empowering myself. My spiritual system is simple" (Interview, 3/7/2018). They think gender equality has existed in the Tai-Yuan community since the early age of their ethnic group. A woman from the focus group said, "We perceive ourselves as equal to men, not lower or higher than them" (Focus group, 4/7/2018).

In addition, it is also noted that technologies have closed gender gaps in several aspects. Unlike in the past when men were mainly in charge of hard work outside the house, and women were responsible mainly for household chores and raising children, now men and women can do work interchangeably due to the help of technologies. In an interview with a Tai-Yuan woman, she said the following:

Before, men were in charge of work such as doing farm work, ploughing, and other hard work outside the house, while women were in charge of household chores involving cooking preparation. Now this has changed. Everyone is equal, and can do what they like to do, and help each other with no distinction between men and women. Women can also plough on the paddy field. There is no longer existence of a gendered labour division as a result of the use of many convenient technologies such as rice grinding machines, cooking facilities, etc. (Interview, 3/7/2018).

From this insight, it can be seen that gender roles and positions are determined by the form of production and livelihood. With this evidence, it could be assumed that those who rely mainly on traditional forms of production in which physical strength are needed

\footnotetext{
${ }^{6}$ Tai Dam is a subgroup of the Lao-Tai linguistic group.
} 
would experience more gender inequality than those who integrate technologies into the production process.

\subsubsection{Challenges facing Tai-Yuan women}

There are three main challenges facing Tai-Yuan women in improving their lives; and in empowering themselves, in particular. The first challenge voiced by the Tai-Yuan women in the focus group is their obligation of supporting their children's education. It is noted that this compromises women's needs. In answering my question, a participant said, "Every two weeks, I receive a call from my children [at school] asking for money" (Interview, 3/7/2018). Children's education is a weighty burden in a family due to the family's low financial income. The women blamed their limited income on the poor local economy. This is considered as their second main challenge. To illustrate this, a statement from the focus group is quoted as follows:

There is no market for our products and produce. We can grow vegetables and much other produce but just for household consumption because there is no market for us to sell them. Even if we were able to sell them, the income would not be enough for family expenses particularly for supporting the children's education because the market price is very low (Focus group, 4/7/2018).

Despite the location of Luang Namtha, which borders with China, and which is close to Thailand, the local economy cannot make a lot of use of this advantage. The focus group participants revealed that the local products could not be exported to China, whereas Chinese products were everywhere in Luang Namtha. In talking about this, a participant said:

We are overloaded with Chinese products while our ones can't be exported to China. Thailand is much better in terms of accepting our products and produce with variety" (Focus group, 4/7/2018).

The third barrier for Tai-Yuan women is the lack of production land. One of the focus group participants voiced that she has no land for producing rice so her family grows rice on the land of another family to receive a half of the produce after each farming season (Focus group, 4/7/2018). It is obvious that this woman was the humblest in the focus group. This implies that it is hard for a woman, who is struggling for survival, to think about empowering herself. When her basic needs have not been satisfied, she is very unlikely to think about meeting strategic needs. 


\subsubsection{Tai-Yuan women's strategies for overcoming the challenges}

The women suggested no specific strategies to overcome the challenges. However, they would like to see job creation in the area, particularly in their village, in order to provide them with income-earning opportunities. An interview participant noted, "I would like to have more attention from the authority and relevant agencies to promoting tourism in our village. This would create more jobs for us" (Interview, 3/7/2018). Another woman suggested that regular meetings of the females in the village would provide a knowledge and information sharing and discussion facility for women (Interview, 3/7/2018).

\subsection{Namdaeng Tai Village findings}

\subsubsection{Hmong women's perceptions of empowerment}

The Hmong women in the focus group agreed that their empowerment means being respected by men. This is explained in that women virtually have no dignity in the family and community due to male dominance and resistance. A woman in the focus group put it that "Men often believe that women can't do anything without men" (Focus group, $5 / 7 / 2018$ ). Although the wife is the breadwinner in the family, the husband wants to be the head in order for the wife to look up to him. Therefore, women have to be patient and forgiving in order to keep family harmony. A woman said "We [women] insist on being a good wife, even for a life time, despite our husband's disrespect of us" (Focus group, 5/7/2018). To further illustrate this, a participant is quoted as follows:

The money deposited at the VB even in the wife's name is also considered to be the husband's money. This is why we [women] don't see any changes in ourselves from our VB membership (Focus group, 5/7/2018).

This indicates that the women would feel their VB membership useful if they faced no male oppression. They asserted that their lives would be so much better if men showed respect to them. Another focus group participant said the following:

Women rarely receive attention and respect from men, particularly when we go to a party or event with our husbands. They dismiss us and go with other girls or friends until late at night. This makes us feel valueless in the family (Focus group, 4/7/2018).

These experiences encourage the Hmong women to hold a belief that women would be empowered if men changed their perceptions and showed respect to them. 


\subsubsection{Savings habit formation with the VB}

The establishment of the VB has enabled several households to start saving. Without it, the villagers have no place to save their money, and have no courage to gain a savings habit. In answering my interview questions, a woman put it that "VB helps me save money. Without it, my family can't save" (Interview, 3/7/2018). She revealed further that her husband had now changed his habit from loose to tight spending. He now gave more consideration before spending money compared to the past. The formation of a savings habit is also seen as necessary among elderly women. In an interview with an elderly woman, she said her VB membership enables her to save some money for her own needs, hopefully for helping her children provide for her own funeral (Interview, 3/7/2018). This is a common concern among Hmong people, particularly among the elderly, who are worried that their own funerals may create burdens and debt to their own children, given that a traditional funeral in Hmong culture costs a lot.

\subsubsection{Limited benefits of the VB}

The interviewees do not think they benefit much from the VB. Those who benefit the most from the VB are the poorest and those who are experiencing emergency situations. The interview participants revealed that there have been a few cases of death in which people needed money to cover the funeral costs. In an interview, a woman said, "The VB would be helpful for me in the future if I am in difficulty or have an emergency" (Interview, 3/7/2018). The participant continued as follows:

I have never borrowed money from the VB because I have no business, and don't know where I should make an investment. I am just a normal person living a life as a normal farmer (Interview, 3/7/2018).

Those who are better off economically do not place importance on the VB. An interview participant who has a household business of trading forest products notes this:

I am economically well off so the VB does not really help me much. I may need to ask the VB for a loan if my business grows much more and needs much bigger funding, but for now I just rely on my own funds. I also have a warm family. I and my husband have never had any conflict so I am happy with my life (Interview, 3/7/2018).

It is also noted that both VBC and ordinary VB members have similar experiences of the VB. One of the VBC members replied in the following way: 
My participation in the VB has no effect on my feelings. I feel as if I have not changed at all because of VB. I think the villagers continue to see me as the same person as before (Interview, 3/7/2018).

This is very similar to what an ordinary VB woman said: "I just go with the flow. I saw they were happy after participating in the VB so I decided to do the same" (Interview, 3/7/2018).

Therefore, it is seen that the villagers consider the VB as a last resort for when they have no way out of difficulties. It is the lack of investments and businesses among the villagers that discourage them from making use of the financial sources. Those who are economically well off do not have a need to use credit at the VB. Both those who have VBC membership and VB ordinary membership have the same reaction to the VB.

\subsubsection{Hope creation with the VB}

Hmong women are facing both psychological and physical poverty. Many of them face men's disrespect and ill treatment, while some others are experiencing traditional discrimination which confines them in hopelessness and with no way out of poverty. The establishment of the VB gives them a new hope which inspires them to keep on going and living. One interviewee, with a strong personality and clear voice, expressed:

Despite the ignorance of family and helplessness of my husband, I am determined to be successful in the future. I am going to expand my small business [a small shop at home selling consumption goods and several simple household use items]. I saw that those who participate in the VB have a better look and more opportunities so I decided to get involved too (Interview, 3/7/2018).

The helplessness of her husband encouraged her to take the lead in her family, because she does not want to see her family fail and be looked down on by the community. To illustrate this, her story is quoted as follows:

I used to look up to my husband and let him lead the family, but I felt that the family was lagging behind others, so I decided to take over his position. Now the family is much better. Otherwise, my family would have gone nowhere because he likes to spend money on unimportant things such as alcohol, tobacco, and drugs. If I had not been decisive, my family would have collapsed. When he was leading the family, we had many debts (Interview, 3/7/2018). 
In addition, she has been facing traditional discrimination, despite her determination. This woman and her husband have been living together for several years, with their own two children, but this couple have not been married officially and traditionally. Her husband is addicted to drugs and has become helpless. Without an official marriage, she is disadvantaged in many aspects. In talking about this, she said the following:

I am sad that I am not like others. I don't see myself as being as valuable as others because I haven't been officially and traditionally married to my husband. This really upsets me. I am doing great things in society to help others but I am not appreciated for that because my relationship with my husband has not been traditionally recognised. One time, at my father-inlaw's funeral, I bought a pig with 2 million Kip [about 250 US dollars] to help lift my in-laws' burdens at the funeral, but they said my contribution was not counted, and they viewed the pig as my intention to win a place in my in-laws' family. I couldn't do anything but burst into tears (Interview, 3/7/2018).

In Hmong culture, when an old person passes away, his or her children and offspring will bring properties such as silver and golden papers, pigs, cattle, and cooked chickens to help cover the funeral costs and offer to the dead in exchange for good luck and blessings. Therefore, in this woman's case, her in-laws discriminated against her partly due to the belief that her pig would not be accepted by the dead's spirit because she has not been traditionally recognised by the in-law family's spirits. In addition, her husband is not respected in the family and community so that this affects the family's and community's views towards herself as well. To illustrate this point, an excerpt from another Hmong woman's interview is quoted: "People will disrespect you [women] if your husband dies or you get divorced. People respecting the husband means they also respect the wife" (Interview, 3/7/2018).

These excerpts of evidence show that the presence of the VB is crucial for Hmong women to make a living and be financially independent in order to avoid being disadvantaged by traditional beliefs and cultural practices. With the women's determination, they will be able to overcome those barriers, as the woman discussed above noted:

I will fight for a better life despite my husband's helplessness, and people's negative thinking about me. I have a plan to expand my shop as this is a strategic location [next to a path to the village primary school]. I am doing my best to improve my life and the lives of my children in order to counter the suffering I have from my husband. I may have fears in doing something new but I believe that, by doing my best, if I can achieve even 
a half of my expectations; that would be enough to feed my children (Interview, 3/7/2018).

Therefore, with limited mobility and economic poverty among the women, the VB is an ideal option for them to start creating and determining their own future.

\subsubsection{Challenges facing Hmong women}

Some barriers have been mentioned earlier, but again this subsection extends on the findings regarding the barriers which keep preventing Hmong women from having the same status as men in family and society.

\section{Limited mobility}

Hmong women usually stay at home, and travel just within the village area. This restricts women's development. A Hmong woman voiced that they are not confident to travel to other cities so that their knowledge is limited to their area. She is quoted: "I have no idea about how to approach any offices or to talk to any office people" (Interview, 3/7/2018). One of the reasons for women's limited mobility, even within the village area, is male resistance. Another interviewee put it in the following way:

Any training for women can cause conflicts between them and their husbands because men are not happy with their wives' participation in any programme. We [women] understand our husbands, but any time they get angry with us, we are sure that they might misunderstand us (Interview, 3/7/2018).

However, the woman revealed that men's control over women's mobility is a sign of love and care. Once this control no longer exists, it would mean the man no longer loves and cares for the woman. Therefore, instead of acting against men's will, the women are happy with male resistance, and consider it as good for them [women].

\section{To be a "good wife"}

When Hmong women decide to get married, they bear in mind the task of being a "good wife" or a "good daughter-in-law". Getting married means getting ready to serve the husband and parents-in-law as well. In an interview with a Hmong woman, she said:

I don't think much about myself. I think I am not as important as my husband so I need to serve him well in order for him to receive respect from people (Interview, 3/7/2018). 
Another participant provided a very similar insight about her feelings towards her husband. She is quoted as follows:

I think the gendered labour division in family is fair because the whole community is doing the same. I rarely get angry with my husband, even when he doesn't want to go to the farm with me early in the morning. I love him so I would like to show other people that we are a true love couple (Interview, 3/7/2018).

The women think that their husbands would look up to them for what they [women] do for them [men]. This means that men's love towards women is conditional. Men will love and care for women as a response to what women do for them. If the woman is not a good wife, he may get married to another woman. This creates a fear in the woman that she may be dismissed by the man if she does not offer enough love and care for him. Therefore, the women tend to maintain men's fidelity by all means.

Even if the husband is helpless, the wife remains determined to manage the family, and take care of the husband and children. This is seen in the story of a participant who confessed: "For me, I think I must take care of my husband, such as buying new clothes for him, as people will judge me for his appearance because they know that he is my husband" (Interview, 3/7/2018).

However, the women revealed that the question of how to be a "good wife" is confusing and challenging sometimes. To illustrate this, a focus group participant is quoted as follows:

Conflicts usually occur when my husband comes from the farm and sees me in the house doing housework; he thinks I spend time at home doing nothing, but actually I have been busy with little things at home all day. That's why conflicts commonly occur in family (Focus group, 4/7/2018).

It is seen that Hmong women's concept of being a "good wife" has compromised their needs and wellbeing. All they do is for the wellbeing of others rather than for their own wellbeing. It can be understood that the women have no personal life but help their husbands live their lives. Women are like the shadow of men. It is true that after marriage, Hmong women's names virtually disappear because they are called after their husbands. 


\section{To be a "good mother"}

Besides being a "good wife", women still need to be a "good mother" for their children. The meaning of being a "good mother" was not explained by the women in many respects but there was a story from a participant who is quoted as follows:

I feel guilt towards my sons because I can't pay their bride price and cover their marriage costs. I blame myself for that because I am poor and a single parent (Interview, 3/7/2018).

This woman is a widow, whose husband died many years ago, so now she lives with her sons and daughters-in-law. She believes that she is crippled by her poverty so that she was unable to pay her sons' bride prices. She said that it is her fault so she feels indebted to her sons. She also believes that, without her husband, she cannot be a good mother. After her husband died, she felt guilt towards her children, and has been looked down on by the villagers. As she said, "People will disrespect you [women] if your husband dies or you get divorced" (Interview, 3/7/2018).

\section{The role of a youngest daughter-in-law}

Normally, the youngest son remains in the family with his parents after his older brothers move out to have their own house after their marriage. He is mainly responsible for the parents. Therefore, his wife needs to serve his parents well. Unluckily, if the daughter-inlaw is not helped by her husband, she will be overwhelmed with family burdens. To illustrate this, an interview participant who is a youngest daughter-in-law revealed as follows:

As the youngest daughter in-law, I feel I handle very many things in the family due to the lack of labour. My husband does not really care about household chores, or taking care of his parents, so I need to make sure everything is done properly as well as ensure my parents-in-law are not starving. I would be very happy if my husband could help me with just a little thing (Interview, 3/7/2018).

This woman has quite a difficult family, particularly after her mother-in-law had a bike accident which is resulted in her being paralysed in the lower part of the body. I had a chance to talk to her mother-in-law and was told that she cannot move anymore due to the uselessness of her two legs. She said that her life now depends totally on her daughterin-law. She is happy that she has a generous daughter-in-law who usually takes care of her (Interview, 3/7/2018). 


\section{A dropped-out school girl}

Most Hmong girls in rural areas tend to get married at an early age. Most of them end their lives in poverty and regret. In an interview with a Hmong woman, she said the following:

I dropped out of school to marry my husband so now I see myself as not as smart as others who finished school. After marriage, I started to engage in farming activities such as growing rice, vegetables, and many others. I want my husband to help me think and do things for the family. I really want to continue learning something new that is useful for me (Interview, 3/7/2018).

Similarly, another Hmong woman voiced that she does not believe that she can catch up with other people who are better than her due to her lack of education and knowledge. In talking about this, she put it in the following way:

My parents didn't allow me to continue school so now I feel so sad for myself. Therefore, I see that my life is obviously lagging behind others. What I can do now is invest in my children in order for them to have a better future, not like me. I am upset as I am poor so nobody respects me (Interview, 3/7/2018).

\section{No way back marriage}

In Hmong tradition, the marriage of a woman is regarded as permanent alienation and as a permanent cutting off from her birth family and clan. She will pick up her husband's clan name and be regarded as being protected by the spirits of her in-laws' family and clan. Therefore, her marriage traditionally and spiritually terminates her return to her birth clan and family. To illustrate this, a Hmong woman is quoted as below:

We [women] don't have many choices after marriage. We will only return to stay with our birth parents if our husbands don't want us any more (Interview, 3/7/2018).

It can be seen that the women not only get married with the men but that they also get married with the men's clan, so any divorce with or death of their husbands does not necessarily mean they get divorced with or are free from the clan. Therefore, a return to a birth clan and family normally causes confusion spiritually among Hmong women. 


\section{"Women can't do anything"}

It is a common belief in rural Hmong communities that women cannot do anything without men. This has normalised the belief of Hmong women that women are born secondary to men so that there is no way for women to be literally equal to men. In an interview with a Hmong woman, she said the following:

I usually think about doing something new but I am not sure because I am female (Interview, 3/7/2018).

This perception is deeply rooted in most Hmong women, who continue to believe that women are always secondary to men in almost every aspect, including education and working. This can be seen as a reason for the commonness of Hmong girls accepting their parents' requests to drop out of school, or even to sacrifice their education to allow their brother or brothers to be educated. This can also be a reason for early marriage among Hmong girls, as they believe that education will take them nowhere. Their lives will depend on their husbands' wisdom, so, the longer they stay single, the more likely they will be to not be able to find a husband. Therefore, their husbands are superior in life.

\section{"Men are born like that"}

The Hmong participants voiced a common belief that men's thinking cannot be changed. A participant said, "Men are born like that, and they continue to behave in such a way, so changing them is impossible" (Interview, 3/7/2018). This perception women hold disadvantages them in many ways. In the focus group with the Hmong women, a common theme is found in the following quote:

Whatever we [women] think and want can't be done and achieved due to men's resistance. Our dreams just slowly die and disappear, as we think men's perceptions can't be changed. We are just to support men's interests but there is virtually no case where men support women in what they [women] are interested in (Focus group, 4/7/2018).

The focus group participants also revealed that most families are led by men, while some others are led by women. One of the participants said, "My husband normally does nothing until I tell him what to do". It can be seen that women are disadvantaged in either case. Where men head families, men dominate decision-making by excluding women from having an influence in the family. Where women lead families, women are in charge of almost everything in the family without men's help. Therefore, women in either case are disempowered. 


\section{The fear of losing face in borrowing}

The Hmong women maintain a belief that borrowing money from someone or any financial institution would make people look down on them. The women are worried that people would judge them as poor and lazy. A participant is quoted as follows:

I think that if I owe money to someone, it will undermine my dignity. I will feel myself to be an unimportant person (Interview, 3/7/2018).

This is a reason why the women avoid borrowing money from others. They value depositing rather than borrowing, as the former helps improve their self-perception. Another interviewee voiced the following:

I see that those who get involved in the VB have a better image, so I decided to follow them. I decided to deposit money in the VB, but I have never borrowed money from it. I think I will use my own savings to expand my personal business [small shop] (Interview, 3/7/2018).

This woman voiced no intention to apply for credit at the VB; rather she only intends to withdraw her own savings for personal use.

\subsubsection{Hmong women's strategies for overcoming the challenges}

The Hmong women have no specific strategies to overcome these challenges. However, they voiced a hope that the thinking and perceptions of their husbands towards women would change. Therefore, these women proposed that training for men in order to change their thinking, and to increase their understanding about women's needs, be undertaken by the government and any development agencies. In talking about this, participants emphasised the following:

If we [women] go out to socialise with friends, our husbands will be unhappy, so conflict occurs between the wife and husband. This is seen regularly in our community. Moreover, about two thirds of Hmong men don't listen to their wives when being asked to remain at home. Any time when we [women] try to stop them from doing something, conflict occurs immediately (Interview, 3/7/2018).

We [women] need an intervention targeting men in order for them to love and care for women (Focus group, 4/7/2018).

One interviewee also voiced that women, particularly herself, have a strong aspiration of being able to see what people in other cities are doing to improve their lives. In talking about this, she put it as follows: 
We [women] really want to see what people in other places of the country, as well as in the world, are doing to improve their lives. We want to learn from others to reduce our poverty (Interview, 3/7/2018).

It can be seen that the Hmong women live their lives with many barriers, but with no effective strategies to tackle them. Their only idea is to keep hoping and waiting for any interventions from outside.

\subsection{Summary}

In this chapter, I have presented the findings from the fieldwork. This was conducted with VB female members from three ethnic groups, Khmu, Tai-Yuan and Hmong, in three different villages in Namtha district, Luang Namtha province. Each ethnic group provided me with a variety of information and insights about empowerment, the impacts of the VBs, challenges, and strategies, and also provided me with some suggestions. There are a number of similar findings between the three ethnic groups of women, but there are variations also. Both the similarities and differences will inform the discussion in the next chapter about the impacts of the AFP project on women's empowerment, as well as the enablers and barriers that are faced by each ethnic group of women. 


\section{Chapter 6: Discussion of findings}

\subsection{Introduction}

This chapter will discuss the findings from the previous chapter by focusing on some key themes. The key themes are integrated into three different major themes: meanings of empowerment, practical and strategic gender needs, and ethnicity. This is done primarily by comparing and contrasting the key themes from the findings in the previous chapter. The discussion focuses on the main issue of this study, the contribution of microfinance to the personal empowerment of women of different ethnicities in Laos, in order to ensure a clear answer to the central research question is presented in the next chapter.

\subsection{The meanings of empowerment}

The meaning of empowerment for women varies between the three ethnic groups. These meanings are simple; referring to personal pride, men's help and respect. Women's attribution of empowerment to simple and personal reasons is consistent with the findings of a study conducted by Doane and Doneys (2015) on women's empowerment with respect to a handicraft project in northern Laos. The project aimed to empower women through its income-generating activities. The study found that most of the women defined empowerment as the ability to support their family and help others. The women's increase in income and acquisition of knowledge and skills were found to even have changed gender relations in their favour, and then enabled them to develop a sense of confidence and independence. These gains provided the women with a feeling of being able to support others. This implies a sense of agency (see Haggard \& Chambon, 2012), which is one of the core values of personal empowerment (see Rowlands, 1997). However, the women from the handicraft project expressed empowerment in terms of their experiences with the project, whereas those in this research expressed empowerment in terms of aspirations they hoped would be achieved with either the AFP project or any other relevant interventions. The Khmu women want to be proud of themselves in economic terms, but the hoped-for economic wealth must be built upon their own knowledge and actions, rather than achieved through immoral deeds such as cheating and doing illegal trade. They also wish men's contribution to housework. The Tai-Yuan women also emphasised the importance of economic wealth to their own empowerment, but they noticed that empowerment can never be achieved without a sense of sufficiency. Therefore, their empowerment is rather about feeling content with what they currently have, without having aspirations that are too high and too many wants. The Hmong 
women are passively waiting for a change in men's behaviour toward women and family. These differing perceptions of empowerment of the three ethnic groups convey a similar notion of agency deprivation. All of them lack the desire to initiate actions toward overcoming their own limitations and current realities. A sense of agency is fundamental in the empowerment of women as suggested by Kabeer (2005). Moreover, the different meanings of empowerment given by women participating in the handicraft project and the AFP project represent individuals' personal and concrete notions of empowerment which do not usually match with those of policymakers.

Policymakers in Laos focus on empowerment by emphasising the provision of opportunities (Doane \& Doneys, 2015). The use of a Lao term (mob umnat) that is directly translated from the word "empowerment" is avoided because it is sensitive in the country. Therefore, empowerment among the policymakers preferably means providing women with opportunities instead of focusing on the notion of "power" explicitly. This means that the women are supported in terms of capacity building in order for them to be able to help others, and fulfil their social and cultural roles better, rather than supported in terms of working on the power within women themselves (Doane \& Doneys, 2015). Women's duties are more about preserving traditions and cultures so the whole society will be proud of them once their duties are done properly (see LWU, 2015). It is assumed that the avoidance of directly targeting women's power is because changes in the community are tardy and culturally associated, so providing opportunities is a temporary measure (Doane \& Doneys, 2015). It is also assumed that avoiding direct emphasis on women's power means shying away from direct confrontation with the political Party. However, Doane and Doneys (2015) contend that the interactions between various national and international stakeholders have blended the notion of empowerment to be something close to individual self-determination. Notwithstanding this, the circumvention of empowerment approaches in Laos can also be explained, as it is by Rowlands (1997), to be because "power" is perceived as "power over" by which one gains power at the expense of others. This means women's empowerment is achieved against men's loss of power. The AFP project's approach toward involving more women in VBs, particularly in decision-making positions, could be a threat to men. Freeing women from having joint accounts with their husbands could increase tensions between wife and husband. These concerns are obvious among the Hmong women who consider themselves as having little or even no control over their own savings at the VB. Therefore, focusing on providing women with opportunities does not ensure the empowerment of 
women, given that it is more about helping women fulfill their survival needs rather than creating transformative agency in women themselves (Kabeer, 2005).

Despite the controversy of "power", empowerment is believed to start from within individuals (Henry, 2011; Mayoux, 1998; Rappaport, 1987; Rowlands, 1997). It is like a self-awakening rather than something bestowed from others. Therefore, the Department of Foreign Affairs and Trade of the Australian Government (DFAT-AG) argues that women's empowerment in Laos must be measured within "the social context and with invisible factors such as support, respect and ability to contribute, rather than just visible factors such as income, assets or the autonomy one may gain" (DFAT-AG, n.d.). Therefore, measuring changes of women in terms of empowerment at the individual level should be based on culturally associated indicators (Oxaal \& Baden, 1997). A programme may benefit some people but not others (Oxaal \& Baden, 1997; Mayoux, 1998). The TaiYuan women were found to have benefited the most from the project compared to the Khmu and the Hmong who appeared to have gained the least benefits. This can be explained by O'Neill (1977, p. 177) who argues that there is no general equality given that people are not equal in every aspect. By extension, it is hard to decide whether the opportunities are equal or not. According to O'Neill (1977), equality of opportunity can be interpreted in two ways: formal and substantive. Formal equality of opportunity means that equality of opportunity can be achieved by removing legal or quasi-legal obstacles that prevent certain groups of people from having the same opportunities as others. Formal equality of opportunity relates to the rules that govern admission to education, jobs, and promotions that matter. Therefore, by applying the same criteria for everyone, society will be just. Any unequal successes which render winners and losers are justifiable, based on the non-discriminating criteria. On the other hand, the substantive interpretation of equal in opportunity refers to different groups of people being given the opportunity to achieve equal rates of success. Equality in this view is considered to have been achieved once people from different groups have equal rates of success in a particular domain. Therefore, according to this interpretation, it can be seen that the approach of the AFP project, which is focused primarily on providing women from the ethnic groups with similar opportunities, is an approach which aspires to a formal equality of opportunity, and it is unlikely to produce identical results between the ethnic groups. This is because each ethnic group of women face their own unique barriers. The Khmu women are commonly facing health issues, overwhelming family obligations, and economic poverty; whereas the Hmong women are mainly faced with exclusion rooted in 
cultural traditions, male dominance, and weighty family obligations. Unlike these two groups, the Tai-Yuan were found to have faced no gender-based discrimination but only socio-economic barriers. Inequality is seen not only between ethnic groups but also between individual women within the same group. This inequality is further discussed in the following subsection.

\subsection{Practical and strategic gender needs}

Although the AFP project has made progress in gender inclusion, its impacts differ between individual women depending on their level of participation, and on their ethnicity. A common pattern from the findings shows that those who engaged in the project as VBC members expressed having gained more in terms of their strategic interests; compared to those who engaged as ordinary VB members who participated for the purpose of meeting their practical needs. Although meeting practical needs is a priority for women, these needs have no effect on women's courage to resist existing gender norms and subordination; given that women's practical interests are restricted to fulfilling their socially normative roles (Moser, 1993). In contrast, strategic needs are women's interests in greater equality, including changing social norms, and improving their status in society (Moser, 1993). Feminists considered strategic gender needs as women's real interests (Moser, 1989). However, these two types of gender needs are not exclusively distinct from each other, given that women with practical needs, because of their particular societal positions, also have some strategic needs (Rowlands, 1997). This suggests that the needs currently perceived by women are the most urgent and prioritised ones that become apparent. These stronger needs may suppress and prevent weaker needs from being expressed, identified, or even perceived by the women themselves. This is commonly seen among the women in the study, particularly the Khmu and Hmong women who dedicate themselves to their family members such as husbands, children, parents-in-law, as well as brothers-in-law and sisters-in-law. They prioritise family wellbeing and harmony over their own needs and interests. These survival needs and interests of women, rather than strategic ones, drive their participation in the project. However, these practical needs and interests were found to have not been satisfied well by the project due to several factors such as women's limited savings, enthusiasm, and confidence. Moreover, these interests of women weaken their strategic needs and interests then make them invisible. It is similarly noted by Williams et al. (1994) that the failures of many development programmes are caused by having not identified the strategic needs of women. The findings of this research also suggest that those participating in the AFP 
project, and who are focused on their own strategic needs and interests are more likely to be empowered.

However, it cannot be concluded that every woman who is focused on her strategic interests will be empowered as a result of their programme membership. Both practical and strategic gender needs are shaped by specific context (Moser, 1989). People develop their interests and needs in the context of their environment and experiences. Therefore, some interests are similar while others differ. On the other hand, each project has a particular focus which is normally unable to cover all of women's interests. Therefore, project activities might meet some women's strategic needs but not those of others. The various meanings of empowerment given by the women show that their strategic interests differ. The meanings vary according to ethnicity primarily. This is explained by Moser (1989, p. 1803) in that “The strategic gender needs identified to overcome women's subordination will vary depending on the particular cultural and sociopolitical context within which they are formulated".

Since practical gender needs, despite their deprivation of empowering courage, are fundamental in the empowerment process (Chambers, 1989; Kabeer, 2015), strategic gender needs should not be targeted exclusively at the expense of practical gender needs. This can be seen as part of the complexity of empowerment itself, as mentioned by Drolet (2005) and Mayoux (1998). Targeting a type of need alone can be misleading. Focusing on strategic gender needs without meeting practical needs may result in disappointments, such as more burdens and emotional stress for women which can further disempower them (Williams et al., 1994). The findings of this study show that most of the women are struggling to meet their practical needs, such as earning enough income to buy food for family, and to support their children, and younger brothers and sisters. Meeting these needs is hard for them, particularly among the Khmu women given their poor health. Health problems prevent Khmu women from thinking about learning something new that would require much mental concentration, although it could benefit them strategically.

On the other hand, the Hmong women continue to face men's resistance to any training and opportunities that benefit them [women] strategically. A Hmong woman said, "Any training for women can cause conflicts between them and their husbands because men are not happy with their wives' participation in any programme". Moreover, Hmong women are influenced by all sorts of traditional norms such as being a "good wife", "good mother", "good daughter-in-law", and "good tradition follower". If these norms are 
violated, the women will very likely have a negative image of themselves followed by emotional stress and confusion. Therefore, the women's increase in power and control over decision-making and resources in the family may result in conflicts, emotional stress, and even family violence.

Empowerment should be considered at the individual level, and as a process (Oxaal \& Baden, 1997). Targeting women's practical and strategic needs can be seen as different and sequential stages of empowerment, with strategic gender needs as the ultimate stage. This means those stages need to be targeted properly. However, those stages do not necessarily happen in a simple sequence but as a simultaneous progress. Any attempt to generalise the empowerment process would result in differing outcomes between groups of women or even between individuals. Therefore, a single project with a certain structure and focus may not produce similar outcomes across the intended beneficiaries. A single project might only produce similar outcomes if the different components and steps of the empowerment process are tailored to the people's various needs. This also suggests that a single project would not be capable of empowering women unless efforts from all sectors are integrated (Oxaal \& Baden, 1997; Moser, 1989). The complexity of meeting gender needs can also be further explained by looking at ethnic factors as follows.

\subsection{Ethnicity}

It has been discussed earlier in the previous section (section 6.3) that women's level of participation in the AFP project (that is, whether they are an ordinary VB member, or a VBC member) influences their perceived gender needs. Those who have a position in their VB have more opportunities for meeting their strategic needs which encourages them to develop more strategic interests. The main challenges faced by these people, according to GIZ (2018), are identified as capacity and confidence. However, the majority of VB members are those whose participation is at a shallower level. The key challenges faced by this majority were, on the other hand, identified as male dominance followed by capacity (Prochaska, et al., 2012).

Although it is hard to generalise about ethnicity based on these three small case studies, there do appear to be important ethnic issues at work. Some findings are similar whereas the others are different. The findings from this research with the Hmong women show that both the VBC and ordinary VB members expressed similar benefits from the VB towards meeting their practical needs. The patriarchal system is believed by the women to have remarkably hindered them, and worsened their feelings towards their project 
membership as something useless. This can be explained to some extent by classical Marxist feminism which argues that traditional gender roles in the family constitute women's subordination which is later further degraded by capitalism making men superior (Jaggar, 1977). A Hmong woman said the following:

Men desire material things such as cars in order to decorate and promote themselves in society.... For many uneducated men, having a car usually leads them to adulterous affairs or infidelity. This is what we [women] don't want. A materialistic lifestyle is rather men's desire not women's (Interview, 3/7/2018).

Classical Marxist feminists argue that monogamous marriage perpetuates class division within family, in which men have supremacy over property and even children (Jaggar, 1977). Marriage between the wife and husband is considered as a relationship of slavery in which women must maintain fidelity while men do not. Women are exploited by working in low paid jobs, and unpaid domestic chores including taking care of children, the old and the sick. Therefore, classical Marxist feminists believe that women's liberation can be realised with a radical change in the family towards putting women in public industries, and assigning women's domestic duties to the state (Jaggar, 1977). This seems to be appropriate for a state pursuing socialism like Laos. However, socialism remains an ideal conception; the initial model of which was tested and failed in the past, and now requires a diversified approach in which economic liberation is enabled, and individuals are allowed more space to pursue their own interests (Stuart-Fox, 2002). This implies the recognition of women's diverse interests as individuals, rather than common collective goals which are more political in nature. Moreover, the classical Marxist feminist point of view fails to take the effect of the traditional beliefs embedded in women into account. Therefore, a more suitable approach towards liberating the Hmong women from traditional gender relations remains needed if any empowering intervention is to be successful.

In contrast, the findings from the research with Tai-Yuan and Khmu women show similar patterns of benefits from the project, but the former perceive themselves as equal to men whereas the latter perceive themselves as secondary to their male counterparts. However, despite the similarity of the patriarchal system between the Hmong and Khmu, females of the latter ethnicity perceive their subordination as biological. Some statements illustrating common themes from the research with Khmu women are quoted as follows: 
Men and women are equal in the public sphere but not in the family.... Women can't be equal to men in the family.... This is my belief (Interview, 6/7/2018).

We [women] respect men because they do hard work and lead the family (Interview, 6/7/2018).

I feel that my husband does more work than I do even though most housework is done by me.... My housework is much lighter than his so I never get angry with him for not helping me with domestic chores... I am happy with it (7/7/2018).

This can be explained to some extent by radical feminism which questions whether women's oppression is rooted in biological difference. Radical feminism argues that patriarchy is fundamental in women's oppression (Murphy \& Livingstone, 1985). Gendered power relations are a matter of individuals rather than of class distinction (Murphy \& Livingstone, 1985) and patriarchy exists in different cultural and economic settings. This generalisation by radical feminism is partly contradicted by the findings of this research with the Tai-Yuan women who are oppressed by their socioeconomic situation; but with the Hmong women we see oppression arising from traditions and culture. The radical feminist point of view that patriarchy is fundamental in women's oppression suggests that political and economic components are not the key ones (Murphy \& Livingstone, 1985). This does not reflect the reality of Laos where political ideology and economic development remain being questioned regarding gender inclusion. The findings of this study also reveal that education plays a promising role in addressing women's subordination to men. A Khmu woman voiced in the focus group that she has more control over her husband and over economic activities because she has a higher education compared to her husband. This implies that, although biological disadvantage has oppressed many Khmu women, its effect is limited among those who have a better education than their husbands. Despite this, it remains unclear whether equal education between a wife and husband can balance the gendered power relations, or have any other effects on family gendered power dynamics in particular.

These different realities between the ethnic groups indicate women's uneven achievements of the personal empowerment core values. The core values of personal empowerment refer primarily to women's awareness of self, others, and their own environment (Rowlands, 1997). They also include the notion of women's action, known as a "sense of agency" or "power to", which actually helps them make a change in their own empowerment. Rowlands's personal empowerment core values can also be 
understood from the Marxist theory of "contradictions", in which "existing forms of social interaction - property relations as well as values, attitudes, and beliefs - come into conflict with new social relations which are generated by changes in the mode of production" (Jaggar, 1977, p. 24). Given this, women's attainment of the core values is seen as feminist consciousness of the contradictions which victimise them, and then actions are required to emerge accordingly (Jaggar, 1977). It is noted that women face hostile forces from either society, or the system, or simply men (Jaggar, 1977). The Hmong women identified male dominance as hostile power, but they are unconscious about the traditional beliefs that are inherently hindering them. They are facing male resistance as a daily incidence, without questioning the traditional value system underlying such force. In contrast, the Khmu women are unaware of their environment due to their attribution of gender inequality to biological difference which is perceived as just for them. Instead of questioning external factors, the Khmu women believe that their subordination to men is biological and a result of their own fault. However, the Tai-Yuan women were found to be much better in terms of experiencing gender equality. Their better status compared to men is granted mainly with the help of traditional practices giving them more self-confidence, self-worth, sense of 'self' in the wider context, dignity, and sense of agency to some extent. Unlike the Hmong and Khmu, the Tai-Yuan women identified society as the hostile force preventing them from pursuing their own interests.

\subsection{Summary}

In this chapter, I have argued that empowerment means different things to different women; and it means different things across the three ethnic groups of women in this study. These meanings of empowerment were constructed based on women's environment and experiences. However, there is a gap between grassroots women and policymakers in defining empowerment. The chapter continues to argue that equality is impossible. As shown in the study, the project renders differing effects on individuals as well as on different ethnic groups of women. The project tends to help the majority of its members to meet their practical gender needs rather than strategic ones. However, even having a focus on strategic gender interests does not necessarily mean that the project will lead to empowerment, given that different ethnic groups of women have different strategic needs, which cannot be covered by a single project. Therefore, the chapter argues that each group of women is facing their own challenges; some are aware of their own environment while others are not, which entails different levels of achievement towards the personal empowerment core values. 


\section{Chapter 7: Conclusion}

The central objective of this study is to examine the contribution of microfinance to the empowerment of women of different ethnic groups in rural Laos. This is done with an emphasis on the core values of personal empowerment, and with an emphasis on the influence of cultural factors on women's achievement of the core values. The study attempted to understand issues that would have an effect on women's perceptions of empowerment and use of the financial services; in particular issues around policy, gender approaches, and gender relations as they are constructed by traditional beliefs and practices. Current gender approaches of the state and the AFP project, and the three ethnic backgrounds, were examined in order to identify their pros and cons in helping the women to achieve personal empowerment core values.

With the help of feminism, the GAD approach, qualitative methodology, and the personal empowerment analytical framework, the research was able to subject the data from fieldwork to an in-depth analysis from a local perspective at the grassroots level. The analysis was done with an investigation of the national context, current gender inclusion approaches, and the particular ethnic backgrounds of the target participants. By doing this, the research has drawn a picture of what local people perceive as empowerment, and why they have such perceptions. It has revealed the extent to which microfinance has contributed to the empowerment of individual women, as well as to the empowerment of groups of women of different ethnicities. The contribution of microfinance has been explained to some extent by looking at women's environment and life experiences. The particularity of the sociocultural context was found to have a significant influence on women's attitude towards, and progress in, empowering themselves with the help of the microfinance opportunities.

In order to give a clear conclusion to the research, this chapter is organised as follows. First, it recaps the contribution of microfinance to the empowerment of women of different ethnicities by taking sociocultural factors into account. This is done with an integration of material from the findings and the discussion chapters. Secondly, it provides some areas of microfinance that need adjustment and expansion. The chapter also expresses some contributions of the research to academic and practical areas. Of course, research limitations and areas for further study are included in this chapter. The chapter ends with a closing remark from the author. 


\subsection{Contribution of microfinance to the empowerment of women of different ethnicities}

In this research, I have argued that women's achievement of personal empowerment core values is fundamental for the entire empowerment process. This process has been assisted by several microfinance interventions around the world, including in Laos. As well as in its national development plan, Laos has placed an emphasis on gender issues by establishing several legal frameworks, setting gender equality goals and implementation plans, and increasing its international cooperation on gender issues. Women are now provided with more opportunities and are included in several development programmes. This is to ensure women's inclusion in development and their equal treatment. Women are part of the national construction and have a key role in maintaining national culture and tradition. The AFP project, which creates Village Banks, has achieved significant outcomes in gender inclusion, which enable rural women to be financially independent, motivated, and equipped with the knowledge and skills necessary for their own empowerment.

However, these attempts have not had a significant effect on women's personal empowerment core values, which are primary for the whole empowerment process. The national approach is focused primarily on providing women with opportunities (Doane \& Doneys, 2015), while women's normative roles in society are emphasised (see LWU, 2015). Although the AFP project has a more optimistic and promising approach towards promoting women's empowerment core values, its effect is limited. The findings show that normally only those who have a position in VBs are likely to attain some core values of personal empowerment through attainment of their strategic needs. The attainment includes the qualities of self-confidence, self-esteem, sense of 'self' in the wider context, and dignity. However, there is virtually no evidence of women's achievement of a "sense of agency", also known as "power to", which allows women to make changes in society including resistance against social structures (Kabeer, 1998; Rowlands, 1999). Without sense of agency, women are restricted to merely awareness of themselves, others, and their own environment without a willingness to take action. Women in this case remain unempowered (Rowlands, 1997).

The research found that for ordinary VB members the microfinance project tends to meet women's practical needs rather than strategic needs. Women's most common use of the financial services is for household consumption and trading. The poorest tend to benefit 
the most from the services in terms of the availability of finance for emergencies. Although meeting both kinds of gender needs is important, practical needs do not have an empowering effect compared to strategic needs (Drolet, 2005). However, meeting practical needs is fundamental for women to develop their ability to meet their strategic needs. Therefore, targeting both gender needs is necessary, and this cannot be done by a single intervention.

It is, therefore, argued that efforts from all sectors are required toward the empowerment of women (Oxaal \& Baden, 1997; Moser, 1989). Women's needs are diverse; ranging from basic to abstract. They are born unequal and live in different environments. The Hmong women perceived their subordination to men as a result of traditional gender roles. They believe that changes in men's perception of women would give women empowerment. Hmong women are restricted by their traditional roles as a good daughterin-law, good wife, and good mother. They are victims of men's negative perceptions about women, of the marriage tradition, and of the patriarchal value system in which men are worthier than women. On the other hand, the Khmu women perceived their subordination to men as biological. This means they believe that there is no need to challenge existing social norms; rather that women can be empowered simply by men's help. Health problems, which are believed by the women to be a result of their own fault, are the key barriers they perceive to their empowerment. Unlike these two ethnic groups of women, the Tai-Yuan women perceive themselves as equal to men. They believe this gender equality exists inherently in the community due to cultural practices. However, the lack of jobs and of a market are the key barriers they perceive to a better livelihood and self-development.

\subsection{Areas for further expansion of microfinance}

Although the microfinance project has its own approach towards gender inclusion, the approach seems to have been influenced by the national orientation towards providing women with opportunities. This has restricted the microfinance project to focusing on gender ratios, rather than on the quality of women's participation. Current approaches tend to target women's confidence which is not enough. Women need to change their perceptions of themselves (Kabeer, 2005; Mayoux, 1998; Rowlands, 1997). Any package or approach with this agenda is recommended.

I have argued that despite the achievement of some empowerment qualities among some programme members, this achievement is not enough to empower women because the 
empowerment achieved lacks a sense of agency. The power from within women is limited to their awareness of themselves, others, and their environment, without any action taken towards changing their realities in their favour. Women's hesitation to take actions is due to their acceptance of the reality that men cannot be changed, women are born biologically secondary to men, and society offers them virtually no opportunity. This requires gender education for both men and women from a patriarchal background. Gender equality measures taken by the State are not enough. The measures tend to target women's effective agency by which women need to perform better in development and cultural preservation rather than women's transformative agency (Kabeer, 2005). An integration of gender education into the national educational curriculum would be promising. Job creation in local areas is needed so that women do not need to migrate for work. This is because women still need to fulfil their roles in their families.

It is good that microfinance is beneficial in terms of meeting women's practical needs. However, women's needs are diverse and cannot be satisfied with a single intervention. Meeting strategic gender needs requires different initiatives, particularly in respect of maternal healthcare and formal education for rural people, particularly minority women and girls.

\subsection{Research contribution}

This research contributes to policy debate and practice within aspects of the areas of microfinance and women's empowerment in Laos. The evidence with multiethnic groups from this research provides a better understanding of the complexity of gender inequality in the country. This can encourage microfinance programmes and any grassroots-based interventions to shape their approaches towards meeting the needs of particular groups of people. This research helps untangle the persistent challenges in poverty reduction and gender equalisation in rural Laos.

From an international point of view, this research helps reaffirm that local knowledge is important for programme design and implementation. The research also signifies the necessity of targeting the core values of personal empowerment in order to ensure women's empowerment. It adds validity to the personal empowerment conceptual framework. The research reinforces the need to look at relevant components of the framework; specifically, to emphasise that women's empowerment starts from within women themselves (Rowlands, 1997). 
More importantly, this research contributes to the untangling of the failures of many women's empowerment initiatives. It has explained that empowerment cannot be achieved without women doing their own part and taking action against existing social structures and values. The research helps increase the understanding of women's needs which are complex and challenging for many development interventions.

\subsection{Research limitation and areas for further study}

Despite the effort put into this research, there are weaknesses and areas for further study as follows. With three months of fieldwork, this research could not explore the depth of the research problem as much as it could be explored. The timeframe did not allow the researcher to go deeper into the women's lives and reality. Interviews and focus groups alone may not be able to capture women's realities from various perspectives. If the fieldwork had been longer, an ethnographic method would have been used to better explore each ethnic group of women. Moreover, if a mixed methods approach had been used, it would have enriched the data, and enabled a more in-depth analysis of women's empowerment and microfinance. On the other hand, there has been little literature about microfinance and women's empowerment in the country. It is even difficult to find the information online given that most of it is in hard copy form. Moreover, the lack of an interdisciplinary approach hinders the analysis and interpretation of the data. In addition, it is hard to generalise about ethnicity based on the three case studies of this research.

This research has identified some areas that need further investigation. Firstly, as this research is focused on women's point of view, it would be interesting to explore men's perception of women and gender equality. This would paint a better picture of women's empowerment in the country. Secondly, as there is evidence that higher education of women can help improve gender relations in the family, it would be interesting to explore how education can weaken the effect of traditional beliefs and cultural values on gender relations.

\subsection{In closing}

This research tries to understand the effect of microfinance on women with a look at traditions and cultural aspects that might have a significant role in women's utilisation of microfinance. I have found that the effect of microfinance on women is limited and insufficient to ignite the power within women themselves. This is because women are facing differing challenges depending on their ethnic background, their self-perception, 
and on local socioeconomic conditions. The study has argued that women's empowerment is a complicated process that requires multiple approaches from relevant parties. 


\section{References}

ADB \& World Bank. (2012). Country gender assessment for Lao PDR: Reducing vulnerability and increasing opportunity. Retrieved from https://openknowledge.worldbank.org/handle/10986/16511

Ahmed, S. M., Chowdhury, M., \& Bhuiya, A. (2001). Micro-credit and emotional wellbeing: Experience of poor rural women from Matlab, Bangladesh. World Development, 29(11), 1957-1966.

Alsop, R., Bertelsen, M., \& Holland, J. (2006). Empowerment in practice: From analysis to implementation. Washington, DC: Poverty Reduction Group, World Bank.

Alcoff, L., \& Potter, E. (1993). Introduction: When feminisms intersect epistemology. In L. Alcoff \& E. Potter (Eds.), Feminist epistemologies (pp. 1-14). New York: Routledge.

Anonymous. (2000). Ethnic classification list. Vientiane, Laos.

Australian National University. (n.d.). Major towns, roads and rivers of Laos. Retrieved from CartoGIS, College of Asia and the Pacific, the Australian National University website: http://asiapacific.anu.edu.au/mapsonline/base-maps/laos

Baden, S. \& Milward, K. (1995). Gender and poverty (Report No. 30). Brighton: Institute of Development Studies.

Banerjee, A., Duflo, E., Glennerster, R., \& Kinnan, C. (2015). The miracle of microfinance? Evidence from a randomized evaluation. American Economic Journal: Applied Economics, 7(1), 22-53.

Batliwala, S. (1994). The meaning of women's empowerment: New concepts from action. In G. Sen, et al. (Eds), Population policies reconsidered: Health, empowerment, and rights (pp. 127-138). New York: Harvard University Press.

Boehe, D. M., \& Cruz, L. B. (2013). Gender and microfinance performance: Why does the institutional context matter? World Development, 47(C), 121-135.

Braun, V., \& Clarke, V. (2006). Using thematic analysis in psychology. Qualitative Research in Psychology, 3(2), 77-101.

Brody, C. M., De Hoop, T., Vojtkova, M., Warnock, R., Dunbar, M., Murthy, P., \& Dworkin, S. (2015). Economic self-help group programs for improving women's empowerment: A systematic review. Campbell Systematic Reviews. Retreived from https://touroscholar.touro.edu/cgi/viewcontent.cgi?referer=https://scholar.google .co.nz/scholar?hl=en\&as_sdt=0\%2C5\&q=Economic + selfhelp+group+programs + for + improving + women's+empowerment $\% 3 \mathrm{~A}+\mathrm{A}+$ syste matic + review $\&$ btnG $=\&$ httpsredir $=1 \&$ article $=1020 \&$ context $=$ cehs_pubs

Burton, M. D. N. (2015, October 19). Self-confidence versus self-esteem [Blog post]. Retrieved from https://www.psychologytoday.com/us/blog/hide-andseek/201510/self-confidence-versus-self-esteem

Buvinic, M. (1983). Women's issues in Third World poverty: A policy analysis. Baltimore: Johns Hopkins University Press.

Carli, L. (1999). Gender, Interpersonal Power, and Social Influence. Journal of Social Issues, 55(1), 81-99. 
Chambers, R. (1989). Editorial introduction: Vulnerability, coping and policy. IDS Bulletin, 20(2), 1-7.

Cheston, S., \& Kuhn, L. (2002). Empowering women through microfinance. Draft, Opportunity International, 64. Retrieved from

https://s3.amazonaws.com/academia.edu.documents/5353418/empowerment.pdf ?AWSAccessKeyId=AKIAIWOWYYGZ2Y53UL3A\&Expires $=1546468096 \& S$ ignature $=$ bvWLITgvYf6Eh4IXcd0c\%2FaBqCCA\%3D\&response-contentdisposition=inline $\% 3 \mathrm{~B} \% 20$ filename $\% 3 \mathrm{DEmpowering}$ _women_through_microfi nance.pdf

CLFNC. (2017). Disclosure of Hmong culture and tradition in Laos. Vientiane:

CLFNC, Department of Ethnic Affairs (DEA).

Code, L. (2006). Ecological thinking: The politics of epistemic location. Oxford: Oxford University Press.

Connel, D. (1999). Participatory development: An approach sensitive to class and gender. In D. Eade (Ed.), Development with Women (pp. 77-93). Oxford: Oxfam GB.

Crang, M., \& Cook, I. (2007). Doing ethnographies. London: Sage Publications.

Creswell, J. W. (Ed.). (2003). Research design: Qualitative, quantitative, and mixed methods approaches (2nd ed.). Thousand Oaks: Sage Publications.

Creswell, J. W. (2014). Research design: Qualitative, quantitative, and mixed methods approaches. London: Sage Publications.

DFAT-AG. (n.d.). Empowerment and security of low-income women in Laos [Policy brief]. Retrieved from https://dfat.gov.au/aboutus/publications/Documents/mekong-economic-social-issues-affecting-lowincome-women-policy-brief-laos.pdf

Doane, D. L., \& Doneys, P. (2015). Lost in translation? Gender and economic empowerment in the Greater Mekong Sub-region. In R. Lund, P. Doneys, \& B. Resurreccion (Eds), Gender entanglements: Revisiting gender in a rapidly changing Asia (pp. 69-95). Copenhagen: NIAS Press.

Drolet, J. (2005). Women and micro credit: Towards an understanding of women's experiences in Cairo, Egypt (Doctoral dissertation). Retrieved from https://search-proquest-com.helicon.vuw.ac.nz/docview/304933812?pqorigsite $=$ primo

Drolet, J. (2010). Feminist perspectives in development: Implications for women and microcredit. Affilia, 25(3), 212-223.

Fife, W. (2005). Doing fieldwork: Ethnographic methods for research in developing countries and beyond. New York: Palgrave Macmillan.

Fisher, C. B., \& Anushko, A. E. (2008). Research ethics in social science. In P. Alasuutari, L. Bickman, \& J. Brannen (Eds.), The SAGE handbook of social research methods (pp. 95-110). Los Angeles: Sage Publications.

Freeman, N. J. (2001). Laos: Timid transition. In J. Funston (Ed.), Government \& politics in southeast Asia. Singapore: Institute of Southeast Asian Studies.

Freire, P. (1970). Pedagogy of the oppressed (M. B. Ramos, Trans.). New York: Herder and Herder. 
Fuchs, T. (2014). Annual microfinance stakeholder forum 2013/14: Report on project progress [PowerPoint slides]. Retrieved from Deutsche Gesellschaft für Internationale Zusammenarbeit, Gmbh (GIZ) website:

https://www.giz.de/en/downloads/giz2014-en-annual-microfinance-stakeholdermeeting.pdf

Gadamer, H. G. (2004). Truth and method (J. Weinsheimer, \& D. G. Marshall, Trans.Rev. ed.). Retrieved from

https://mvlindsey.files.wordpress.com/2015/08/truth-and-method-gadamer2004.pdf

Garikipati, S. (2008). The impact of lending to women on household vulnerability and women's empowerment: Evidence from India. World Development, 36(12), 26202642.

Geleta, E. B. (2016). The microfinance mirage: The politics of poverty, social capital and women's empowerment in Ethiopia. New York: Routledge.

Gibbs, G. R. (2007). Qualitative research kit: Analyzing qualitative data. London: Sage Publications.

GIZ. (2014). Handbook of operating village banks. Retrieved from https://www.giz.de/en/downloads/giz2014-en-operating-village-bankshandbook-annexes.pdf

GIZ. (2015). Assessing financial literacy in rural Laos: Survey results from the provinces Champasak, Salavan, and Savannaketh. Retrieved from https://www.giz.de/en/downloads/giz2015-en-assessing-financial-literacy-ruralareas-laos.pdf

GIZ. (2017). Microfinance in rural areas - access to finance for the poor (AFP): Fostering local economic development through financial inclusion in rural Laos. Retrieved from https://www.giz.de/en/downloads/ENG\%20AFP\%20Fact\%20Sheet.pdf

GIZ. (2018). Presentation in March 2018: Women's financial inclusion: Access to finance for the poor. Power point slides presented at a meeting at GIZ office, Vientiane, Laos.

Goldman, M. J., \& Little, J. S. (2015). Innovative grassroots NGOs and the complex processes of women's empowerment: An empirical investigation from Northern Tanzania. World Development, 66, 762-777.

Grasswick, H. E. (Ed.) (2011). Feminist epistemology and philosophy of science in knowledge. London: Springer.

Haggard, P., \& Chambon, V. (2012). Sense of agency. Current Biology, 22(10), R390R392.

Hammersley, M., \& Atkinson, P. (2007). Ethnography: Principles in practice. London: Routledge.

Hansen, N. (2015). The development of psychological capacity for action: The empowering effect of a microfinance programme on women in Sri Lanka. Journal of Social Issues, 71(3), 597-613.

Haraway, D. (1988). Situated knowledges: The science question in feminism and the privilege of partial perspective. Feminist Studies, 14(3), 575-599. 
Harding, S. (1991). Whose science? Whose knowledge?: Thinking from women's lives. Ithaca: Cornell University Press.

Hashemi, S. M., Schuler, S. R., \& Riley, A. P. (1996). Rural credit programs and women's empowerment in Bangladesh. World Development, 24(4), 635-653.

Henry, H. M. (2011). Egyptian women and empowerment: A cultural perspective. Women's Studies International Forum, 34(3), 251-259.

Hull, D. L. (2015). Evolutionary epistemology. In J. Wright (Ed.), International encyclopedia of the social and behavioral sciences (2nd ed., pp. 437-440). Oxford: Elsevier.

Inui, M. (2015). Hmong women and education: Challenges for empowerment in the Lao PDR. Hmong Studies Journal, 16(1), 1-24.

Ireson-Doolittle, C., \& Moreno-Black, G. (2004). The Lao: Gender, power, and livelihood. Boulder: Westview Press.

Jaggar, A. (1977). Political philosophies of women's liberation. In M. VetterlingBraggin, F. A. Elliston, \& J. English (Eds), Feminism and philosophy (pp. 5-21). New Jersey: Littlefield, Adams \& Co.

Jhangiani, R., Tarry, H., \& Stagnor, C. (2015). Principles of social psychology (1st international ed.). Retrieved from http://pzacad.pitzer.edu/ hfairchi/courses/Spring2015/Psych\%20103/Principlesof-Social-Psychology-1st-International-Edition-1415042666.pdf

JICA. (2006). Lao PDR: Country gender profile. Retrieved from https://www.jica.go.jp/english/our_work/thematic_issues/gender/background/pd f/e05lao.pdf

Kabeer, N. (1994). Reversed realities: Gender hierarchies in development thought. London: Verso.

Kabeer, N. (1998). Money can't buy me love? Re-evaluating gender, credit and empowerment in rural Bangladesh (IDS Discussion Paper 363). Brighton: Institute of Development Studies.

Kabeer, N. (2005). Gender equality and women's empowerment: A critical analysis of the third millennium development goal 1. Gender \& Development, 13(1), 13-24.

Kabeer, N. (2012). Women's economic empowerment and inclusive growth: Labour markets and enterprise development. International Development Research Centre, 44(10), 1-70.

Kabeer, N. (2015). Gender, poverty, and inequality: A brief history of feminist contributions in the field of international development. Gender \& Development, 23(2), 189-205.

Khadka, N. (2014). Empowerment of women through microfinance in Jabdi village development committee, Nepal (Master's thesis). Retrieved from Norwegian University of Life Sciences website: https://brage.bibsys.no/xmlui/handle/11250/279185

Koczberski, G. (1998). Women in development: A critical analysis. Third World Quarterly, 19(3), 395-410.

Krenz, K., Gilbert, D. J., \& Mandayam, G. (2014). Exploring women's empowerment through "credit-plus" microfinance in India. Affilia, 29(3), 310-325. 
Kvale, S., \& Brinkmann, S. (2009). Interviews: Learning the craft of qualitative research interviewing. Los Angeles: Sage Publications.

LFNC. (2008). The ethnic groups in Lao PDR. Vientiane: LFNC, DEA.

Lao PDR. (1991). The Constitution of the Lao People's Democratic Republic. Retrieved from http://confinder.richmond.edu/admin/docs/laos.pdf

Lao Statistics Bureau. (2016). Results of population and housing census 2015. Retrieved from http://lao.unfpa.org/en/publications/results-population-andhousing-census-2015-english-version

Litosseliti, L. (2003). Using focus groups in research. London: Continuum.

LNTD. (n.d.). Ethnic diversity in Luang Namtha, Laos. Retrieved July 15, 2018, from Luang Namtha Tourism Department website: http://www.tourismlaos.info/luangnamtha/ethnic.htm

Loutfi, M. F. (1987). Rural women: Unequal partners in development. Geneva: International Labour Organisation.

Lucy, D. M., Ghosh, J., \& Kujawa, E. (2008). Empowering women's leadership: A case study of Bangladeshi microcredit business. SAM Advanced Management Journal, 73(4), 31-39,50.

LWU. (2015). The seventh national women's congress. Vientiane: Lao Uniprint Press.

Malhotra, A., \& Schuler, S. R. (2005). Women's empowerment as a variable in international development. In D. Narayan (Ed.), Measuring empowerment: Cross-disciplinary perspectives (pp. 71-88). Washington: World Bank.

Marshall, P. A. (2003). Human subjects protections, institutional review boards, and cultural anthropological research. Anthropological Quarterly, 76(2), 269-285.

Mason, K. O., \& Smith, H. L. (2003). Women's empowerment and social context: Results from five Asian countries. Retrieved from https://pdfs.semanticscholar.org/6842/3faadedb45524c55263554bea269d2f4fb1 5.pdf

Mayoux, L. (1998). Participatory learning for women's empowerment in microfinance programmes: Negotiating complexity, conflict and change. IDS bulletin, 29(4), $39-50$.

Mayoux, L. (2002). Women's empowerment or feminisation of debt?: Towards a new agenda in African microfinance (Report based on a One World Action conference). Retrieved from http://oneworldaction.org.uk/WoEmp.pdf

Meyer, R. L., \& Nagarajan, G. (2006). Microfinance in developing countries: Accomplishments, debates, and future directions. Agricultural Finance Review, 66(2), 167-193.

MFA. (n.d.). Microfinance in Laos. Retrieved July 20, 2018, from Lao Microfinance Association website: https:/www.laomfa.org/about-us/microfinance-in-laos/

Moser, C. O. N. (1989). Gender planning in the Third World: Meeting practical and strategic gender needs. World Development, 17(11), 1799-1825.

Moser, C. O. N. (1993). Gender planning and development: Theory, practice and training. London: Routledge. 
MPI. (2016). Eighth five-year national socio-economic development plan (2016-2020). Retrieved from http://www.la.one.un.org/images/publications/8th_NSEDP_2016-2020.pdf.

Murphy, L., \& Livingstone, J. (1985). Racism and the limits of radical feminism. Race \& Class, 26(4), 61-70.

Nader, Y. F. (2008). Microcredit and the socio-economic wellbeing of women and their families in Cairo. The Journal of Socio-Economics, 37(2), 644-656.

Nanji, S. (2014). Importance of women's empowerment. Pakistan \& Gulf Economist, 33(49), 16-17.

Noreen, S. (2011). Role of microfinance in empowerment of female population of Bahawalpur district. International Proceedings of Economics Development \& Research, 4(20), 318-324.

Ocasio, V. M. (2016). Rural finance schemes miss the target: An empirical analysis of gender and social inequality effects. Journal of Applied Business and Economics, 18(6), 78-93.

O'Leary, Z. (2007). The social science jargon buster: The key terms you need to know. Los Angeles: Sage Publications.

O’Neill, O. (1977). How do we know when opportunities are equal? In M. VetterlingBraggin, F. A. Elliston, \& J. English (Eds), Feminism and philosophy. New Jersey: Littlefield, Adams \& Co.

Oxaal, Z., \& Baden, S. (1997). Gender and empowerment: Definitions, approaches and implications for policy (Report No. 40). Retrieved from http://www.bridge.ids.ac.uk/sites/bridge.ids.ac.uk/files/reports/re40c.pdf

Page, N., \& Czuba, C. E. (1999). Empowerment: What is it? Journal of Extension, 37(5), 1-5.

Panjaitan-Drioadisuryo, R. D., \& Cloud, K. (1999). Gender, self-employment and microcredit programs: An Indonesian case study. The Quarterly Review of Economics and Finance, 39(5), 769-779.

Parse, R. R. (2016). Human dignity: Respect and incivility. Nursing Science Quarterly, $29(4), 261$.

Perkins, D. D., \& Zimmerman, M. A. (1995). Empowerment theory, research, and application. American Journal of Community Psychology, 23(5), 569-579.

Phonesavanh, S. (2015). The impact of microfinance on poverty reduction in Oudomxay, northern province, Lao PDR (Master's thesis). Retrieved from http://r-cube.ritsumei.ac.jp/repo/repository/rcube/7466/51213620.pdf

Premsrirat, S. (2002). Appropriateness in Khmu culture. Mon-Khmer Studies, 32, 117 129.

Presser, H., \& Sen, G. (Eds). (2000). Women's empowerment and demographic processes: Moving beyond Cairo. Oxford: Oxford University Press.

Prochaska, K., Hauger, P., \& Fischer, D. (2012). Rural finance in Laos: GIZ experience in remote rural areas. Vientiane: GIZ.

Rappaport, J. (1987). Terms of empowerment/exemplars of prevention: Toward a theory for community psychology. American Journal of Community Psychology, $15(2), 121-148$. 
Reeves, H., \& Baden, S. (2000). Gender and development: Concepts and definitions (Report No. 55). Brighton: Bridge.

Robinson, M. (2001). Microfinance revolution: Sustainable finance for the poor. Washington: World Bank Publications.

Rogers, B. (2005). The domestication of women: Discrimination in developing societies. London: Routledge.

Rowlands, J. (1997). Questioning empowerment: Working with women in Honduras. Oxford: Oxfam.

Rowlands, J. (1999). Empowerment Examined. In D. Eade (Ed.), Development with women (pp. 141-150). Oxford: Oxfam GB.

Sayvaya, I. (2012). Does microfinance reduce poverty in Lao PDR? Case study of village development funds (VDFs) at Sukhuma district, Champassak province, Lao PDR (Mekong Institute Research Working Paper Series, Paper No. 4). Khon Kaen: Mekong Institute.

Schenk-Sandbergen, L. (2012). The Lao matri-system, empowerment, and globalisation. Journal of Lao Studies, 3(1), 65-90.

Schmidt, L. K. (2006). Understanding hermeneutics. Durham: Acumen Publishing Limited.

Schuler, S. R., Hashemi, S. M., Riley, A. P., \& Akhter, S. (1996). Credit programs, patriarchy and men's violence against women in rural Bangladesh. Social science \& medicine, 43(12), 1729-1742.

Sengsourivong, K., \& Mieno, F. (2014). Impacts of savings and credit union programs on household welfare in Laos: Case study of the Vientiane vicinity during the mid-2000s (Discussion Paper No. 55). Tokyo: Hitotsubashi University Repository.

Siraj, M. (2012). Do microfinance programs benefit women in developing countries? Advancing Women in Leadership, 32, 24-35.

Smith, H. L. (1989). Integrating theory and research on the institutional determinants of fertility. Demography, 26(2), 171-184.

Snow, D. A. (1980). The disengagement process: A neglected problem in participant observation research. Qualitative Sociology, 3(2), 100-122.

St. John, R. B. (1995). New economic order in Indochina. Asian Affairs, 21(4), 227-240.

Stone, L. (2008). Epistemology. In L. M. Given (Ed.), The sage encyclopedia of qualitative research methods (pp. 265-268). Los Angeles: Sage Publications.

Stuart-Fox, M. (2002). Buddhist kingdom, Marxist state: The making of modern Laos (2nd ed.). Bangkok: White Lotus Press.

Stuart-Fox, M. (2007). Laos: Politics in a single-party state. Southeast Asian Affairs (34), 161-180.

Swain, R. B., \& Wallentin, F. Y. (2009). Does microfinance empower women? Evidence from self-help groups in India. International Review of Applied Economics, 23(5), 541-556.

Tesoriero, F. (2006). Strengthening communities through women's self help groups in South India. Community Development Journal, 41(3), 321-333. 
Than, M., \& Tan, L. H. (Eds.). (1997). Laos' dilemmas and options: The challenge of economic transition in the 1990s. Singapore: Institute of Southeast Asian Studies.

Thiel, S. V. (2014). Research methods in public administration and public management. New York: Routledge.

Tinker, I. (1990). Persistent inequalities: Women and world development. New York: Oxford University Press.

UNDP. (1995). Human development report 1995. Retrieved from United Nations Development Programme (UNDP) website: http://hdr.undp.org/sites/default/files/reports/256/hdr_1995_en_complete_nostat s.pdf

UNDP. (2018). Summary of human development indices and indicators: 2018 statistical update. Retrieved from UNDP website:

http://hdr.undp.org/sites/default/files/2018_summary_human_development_stati stical_update_en.pdf

United Nations. (2001). Guidelines on women's empowerment for the UN resident coordinator system. Retrieved from the United Nations website: http://www.un.org/popin/unfpa/taskforce/guide/iatfwemp.gdl.html

United Nations. (2015). Country analysis report: Lao PDR. Retrieved from UNDP website:

http://www.la.undp.org/content/dam/laopdr/docs/Reports\%20and\%20publicatio ns/2015/Country\%20Analysis\%20Report\%20Lao\%20PDR\%202015.pdf

Visvanathan, L., Dugan, L., Nisonoff, \& Wiegersma, N. (Eds.). (1997). The women, gender \& development reader. London: Zed Books.

Williams, S., Seed, J., \& Mwau, A. (1994). The Oxfam gender training manual. Retrieved from the Oxfam website: https://oxfamilibrary.openrepository.com/bitstream/handle/10546/141359/bkgtm-8-gender-sensitive-appraisal-010194-en.pdf?sequence=8\&isAllowed=y

Willig, C. (2014). Interpretation and analysis. In U. Flick (Ed.), The sage handbook of qualitative data analysis (pp. 136-150). London: Sage Publications.

World Bank. (1996). Sustainable banking with the poor: A worldwide inventory of microfinance institutions. Retrieved from the World Bank website: http://documents.worldbank.org/curated/en/184061468739265775/pdf/multipage.pdf

World Bank. (2018). Lao PDR: Overview. Retrieved from the World Bank website: http://www.worldbank.org/en/country/lao/overview

Yin, R. K. (2016). Qualitative research from start to finish (2nd ed.). New York: Guilford Publications.

Zimmerman, M. A. (1990). Taking aim on empowerment research: On the distinction between individual and psychological conceptions. American Journal of Community Psychology, 18(1), 169-177. 


\section{Appendices}

\section{Appendix 1.1. Information sheet for interviews - Village Bank members}

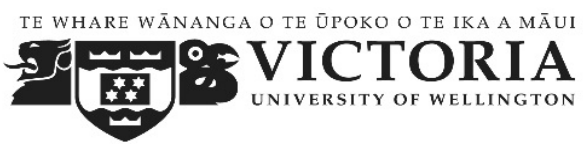

Research Understanding the influence of cultural factors on the perceptions of title: empowerment and the use of microfinance for empowerment of ethnic women in northern Laos.

Researcher: $\quad$ Souasong Tongnamavong

Supervisor: Prof. John Overton

Sabaidee and thank you for your interest in this research project. Please read the information below to gain a better understanding of the project. After reading this paper, please feel free to decide whether you would like to participate in this project or not. If you decide to participate, thank you so much for your time and contribution. If you decide not to participate, thank you for your consideration of my request and I wish you all the best with your studies or work.

\section{Who am I?}

My name is Souasong Tongnamavong, a Master's student of Development Studies at Victoria University of Wellington, New Zealand. As part of the university's requirements for the completion of my studies, I am doing a research project titled: "Understanding the influence of cultural factors on the perceptions of empowerment and the use of microfinance for empowerment of ethnic women in northern Laos".

I have chosen this topic because I am passionate about exploring how ethnic women perceive empowerment differently and how their cultural backgrounds have an influence on their use of microfinance to empower themselves. I hope that the findings of this research will not only serve my academic interest, but will also inform development theories and practices.

This research project has been reviewed and approved by the Human Ethics Committee of the Victoria University of Wellington, New Zealand.

\section{What is the aim of this project?}

This research aims to:

- explore the perceptions of different ethnic women about empowerment;

- explore cultural factors that have an influence on women's use of microfinance for empowerment;

- find out challenges and opportunities in the use of microfinance among different ethnic women, and the gap that current gender-based development approaches in rural Laos need to fill.

\section{How will I proceed?}

If you agree to participate, I will invite you for face-to-face interview. The interview will take between 30 to 90 minutes. You will be asked about: 
- Your ethnic background: ethnic group, gender relations in your culture, the roles of women and men in your family;

- Your perceptions towards empowerment;

- Your perspectives on how the microfinance scheme has changed your life and relations with your husband;

- Your views about factors from the microfinance scheme that contribute to your success;

- Your views about any cultural factors that have influence on the use of microfinance;

- Your opinion about any other challenges facing you in making use of the microfinance scheme;

- Your strategies to overcome those challenges.

The time and location for interview totally depend on your decision. You can feel free to choose when and where you feel most comfortable to talk. However, please note that a safe and uninterrupted place is highly desired given that confidentiality of the interview needs to be guaranteed. You can feel free to invite anyone who you think his or her accompany will make you feel relaxed and confident to talk in the interview. However, that person will also need to keep confidentiality of the interview. With your permission, I will make audio recordings and take notes of our discussions. You can stop the interview at any time or refuse to answer any question without giving a reason.

\section{What will happen after the interview?}

Your information will be confidential. All the records and notes from the discussions will be kept securely. I will transcribe and analyse the information. Only my supervisor and I will read the notes or transcripts of the interview. If data transcribers are required, they will be asked to sign a consent form which means that your information will not be disclosed to any other third parties.

Your name will not be mentioned in any reports, unless otherwise agreed. The interview transcripts, summaries, and recordings will be kept securely and destroyed after five years since the end of the research.

\section{What will the project produce?}

The final report (thesis) of this project will be published and held at Victoria University of Wellington library. I may also use the results of my research for conference presentations and academic reports. I will take care of your information and not identify you in any presentation or report.

\section{What if you would like to withdraw from taking part of the project?}

You can withdraw from the research up to four weeks after the interview. If you withdraw, the information you provided will be destroyed or returned to you.

\section{If you accept this invitation, what are your rights as a research participant?}

You do not have to accept this invitation if you don't want to. If you decide to participate, you have the right to:

- choose not to answer any question;

- ask for the recorder to be turned off at any time during the interview;

- withdraw from the study up to four weeks after your interview;

- ask any questions about the study at any time;

- receive a copy of your interview recording (if it is recorded); 
- read over and make comments on a written summary of your interview;

- agree on another name for me to use rather than your real name;

- be able to read any reports of this research by emailing the researcher to request a copy.

\section{If you have any questions or problems, who can you contact?}

If you have any questions, either now or in the future, please feel free to contact either:

Student:

Name: Souasong Tongnamavong

Course: Master of Development Role: Studies

School: School of Geography, Environment, and Earth Sciences

Phone: $\quad$ +856 2099263532 (Laos) +640221294723 (New Zealand)

Email: $\quad$ tongnasoua@myvuw.ac.nz

Facebook: Souasong Tongnamavong

\section{Your village head:}

In case you cannot contact me directly, your village head will help you contact me.

\section{Supervisor:}

Name: $\quad$ Prof. John Overton

Course coordinator and lecturer of Development Studies

School: School of Geography, Environment, and Earth Sciences

Phone: $\quad+64044635281$

Email:

\section{John.overton@,vuw.ac.nz}

\section{Human Ethics Committee information}

If you have any concerns about the ethical conduct of the research you may contact the Victoria University HEC Convenor: Dr. Judith Loveridge. Email: hec@vuw.ac.nz or phone: +64 44636028 . 


\title{
Appendix 1.2. Information sheet for interviews - GIZ Laos officers
}

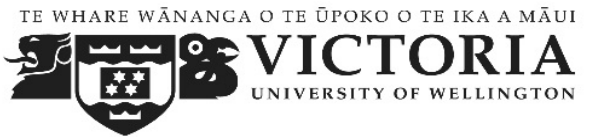

\author{
Research Understanding the influence of cultural factors on the perceptions of \\ title: empowerment and the use of microfinance for empowerment of \\ ethnic women in northern Laos. \\ Researcher: Souasong Tongnamavong \\ Supervisor: Prof. John Overton
}

Sabaidee and thank you for your interest in this research project. Please read the information below to gain a better understanding of the project. After reading this paper, please feel free to decide whether you would like to participate in this project or not. If you decide to participate, thank you so much for your time and contribution. If you decide not to participate, thank you for your consideration of my request and I wish you all the best with your studies or work.

\section{Who am I?}

My name is Souasong Tongnamavong, a Master's student of Development Studies at Victoria University of Wellington, New Zealand. As part of the university's requirements for the completion of my studies, I am doing a research project titled: "Understanding the influence of cultural factors on the perceptions of empowerment and the use of microfinance for empowerment of ethnic women in northern Laos".

I have chosen this topic because I am passionate about exploring how ethnic women perceive empowerment differently and how their cultural backgrounds have an influence on their use of microfinance to empower themselves. I hope that the findings of this research will not only serve my academic interest, but will also inform development theories and practices.

This research project has been reviewed and approved by the Human Ethics Committee of the Victoria University of Wellington, New Zealand.

\section{What is the aim of this project?}

This research aims to:

- explore the perceptions of different ethnic women about empowerment;

- explore cultural factors that have an influence on women's use of microfinance for empowerment;

- find out challenges and opportunities in the use of microfinance among different ethnic women, and the gap that current gender-based development approaches in rural Laos need to fill.

\section{How will I proceed?}

If you agree to participate, I will invite you for face-to-face interview. The interview will take between 30 to 90 minutes. You will be asked about:

- Your view towards ethnic women's perceptions of empowerment;

- Your role in influencing and creating conditions for the empowerment of ethnic women; 
- Your observations of women's participation in village banks;

- Your strategies to help ethnic women achieve their empowerment aspirations.

The time and location for interview totally depend on your decision. You can feel free to choose when and where you feel most comfortable to talk. With your permission, I will make audio recordings and take notes of our discussions. You can stop the interview at any time or refuse to answer any question without giving a reason.

\section{What will happen after the interview?}

Your information will be confidential. All the records and notes from the discussions will be kept securely. I will transcribe and analyse the information. Only my supervisor and I will read the notes or transcripts of the interview. If data transcribers are required, they will be asked to sign a consent form which means that your information will not be disclosed to any other third parties.

Your name will not be mentioned in any reports, unless otherwise agreed. The interview transcripts, summaries, and recordings will be kept securely and destroyed after five years since the end of the research.

\section{What will the project produce?}

The final report (thesis) of this project will be published and held at Victoria University of Wellington library. I may also use the results of my research for conference presentations and academic reports. I will take care of your information and not identify you in any presentation or report.

What if you would like to withdraw from taking part of the project?

You can withdraw from the research up to four weeks after the interview. If you withdraw, the information you provided will be destroyed or returned to you.

\section{If you accept this invitation, what are your rights as a research participant?}

You do not have to accept this invitation if you don't want to. If you decide to participate, you have the right to:

- choose not to answer any question;

- ask for the recorder to be turned off at any time during the interview;

- withdraw from the study up to four weeks after your interview;

- ask any questions about the study at any time;

- receive a copy of your interview recording (if it is recorded);

- read over and make comments on a written summary of your interview;

- agree on another name for me to use rather than your real name;

- be able to read any reports of this research by emailing the researcher to request a copy.

If you have any questions or problems, who can you contact?

If you have any questions, either now or in the future, please feel free to contact either:

\section{Student:}

Name:

Souasong Tongnamavong

Course:

Master of Development Role:
Studies

Supervisor:

Name:

Prof. John Overton

Course coordinator and lecturer of Development Studies 


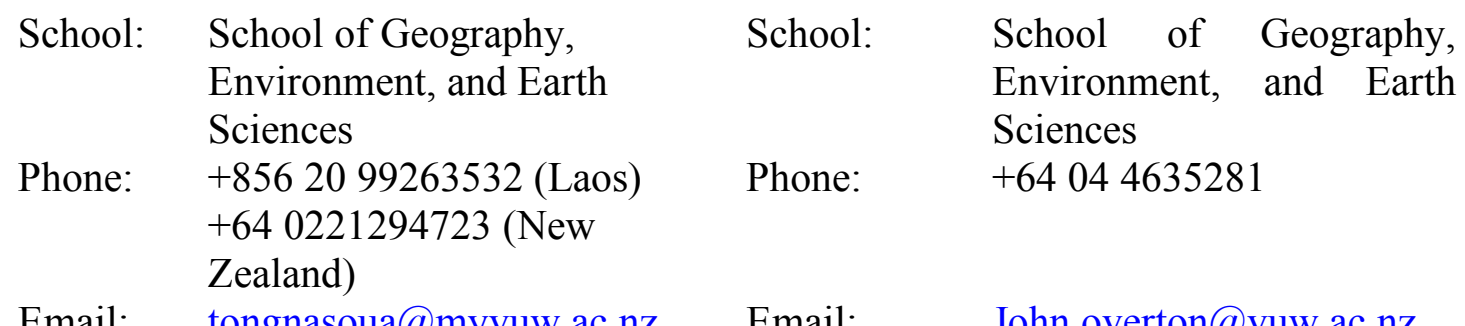

Email: tongnasoua@myvuw.ac.nz_Email: $\quad$ John.overton@,vuw.ac.nz

\section{Human Ethics Committee information}

If you have any concerns about the ethical conduct of the research you may contact the Victoria University HEC Convenor: Dr. Judith Loveridge. Email: hec@vuw.ac.nz or phone: +64 44636028 . 


\title{
Appendix 1.3. Information sheet for interviews - Officers of Bank of Laos
}

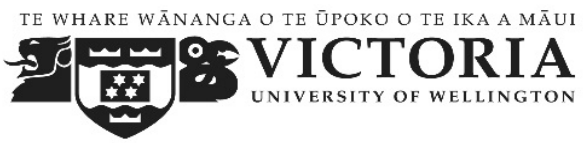

\begin{abstract}
Research Understanding the influence of cultural factors on the perceptions of title: empowerment and the use of microfinance for empowerment of ethnic women in northern Laos.

Researcher: $\quad$ Souasong Tongnamavong

Supervisor: Prof. John Overton
\end{abstract}

Sabaidee and thank you for your interest in this research project. Please read the information below to gain a better understanding of the project. After reading this paper, please feel free to decide whether you would like to participate in this project or not. If you decide to participate, thank you so much for your time and contribution. If you decide not to participate, thank you for your consideration of my request and I wish you all the best with your studies or work.

\section{Who am I?}

My name is Souasong Tongnamavong, a Master's student of Development Studies at Victoria University of Wellington, New Zealand. As part of the university's requirements for the completion of my studies, I am doing a research project titled: "Understanding the influence of cultural factors on the perceptions of empowerment and the use of microfinance for empowerment of ethnic women in northern Laos".

I have chosen this topic because I am passionate about exploring how ethnic women perceive empowerment differently and how their cultural backgrounds have an influence on their use of microfinance to empower themselves. I hope that the findings of this research will not only serve my academic interest, but will also inform development theories and practices.

This research project has been reviewed and approved by the Human Ethics Committee of the Victoria University of Wellington, New Zealand.

What is the aim of this project?

This research aims to:

- explore the perceptions of different ethnic women about empowerment;

- explore cultural factors that have an influence on women's use of microfinance for empowerment;

- find out challenges and opportunities in the use of microfinance among different ethnic women, and the gap that current gender-based development approaches in rural Laos need to fill.

\section{How will I proceed?}

If you agree to participate, I will invite you for face-to-face interview. The interview will take between 30 to 90 minutes. You will be asked about:

- Your view towards microfinance in Laos;

- Your view towards culture and its influence on the empowerment perceptions of ethnic women; 
- Your opinion about the influence of culture on women's use of microfinance for empowerment;

- Your strategies and interventions to help ethnic women achieve their empowerment aspirations.

- The lessons that you have learned from your experience working on women's empowerment.

The time and location for interview totally depend on your decision. You can feel free to choose when and where you feel most comfortable to talk. With your permission, I will make audio recordings and take notes of our discussions. You can stop the interview at any time or refuse to answer any question without giving a reason.

\section{What will happen after the interview?}

Your information will be confidential. All the records and notes from the discussions will be kept securely. I will transcribe and analyse the information. Only my supervisor and I will read the notes or transcripts of the interview. If data transcribers are required, they will be asked to sign a consent form which means that your information will not be disclosed to any other third parties.

Your name will not be mentioned in any reports, unless otherwise agreed. The interview transcripts, summaries, and recordings will be kept securely and destroyed after five years since the end of the research.

\section{What will the project produce?}

The final report (thesis) of this project will be published and held at Victoria University of Wellington library. I may also use the results of my research for conference presentations and academic reports. I will take care of your information and not identify you in any presentation or report.

What if you would like to withdraw from taking part of the project?

You can withdraw from the research up to four weeks after the interview. If you withdraw, the information you provided will be destroyed or returned to you.

\section{If you accept this invitation, what are your rights as a research participant?}

You do not have to accept this invitation if you don't want to. If you decide to participate, you have the right to:

- choose not to answer any question;

- ask for the recorder to be turned off at any time during the interview;

- withdraw from the study up to four weeks after your interview;

- ask any questions about the study at any time;

- receive a copy of your interview recording (if it is recorded);

- read over and make comments on a written summary of your interview;

- agree on another name for me to use rather than your real name;

- be able to read any reports of this research by emailing the researcher to request a copy.

\section{If you have any questions or problems, who can you contact?}

If you have any questions, either now or in the future, please feel free to contact either:

Student:

Name:
Souasong Tongnamavong

\section{Supervisor:}

Name: $\quad$ Prof. John Overton 


\begin{tabular}{|c|c|c|c|}
\hline Course: & of Development & Role: & $\begin{array}{l}\text { Course coordinator and } \\
\text { lecturer of Development } \\
\text { Studies }\end{array}$ \\
\hline School: & $\begin{array}{l}\text { School of Geography, } \\
\text { Environment, and Earth } \\
\text { Sciences }\end{array}$ & School: & $\begin{array}{l}\text { School of Geography, } \\
\text { Environment, and Earth } \\
\text { Sciences }\end{array}$ \\
\hline Phone: & $\begin{array}{l}+8562099263532(\mathrm{Laos}) \\
+640221294723(\mathrm{New} \\
\text { Zealand) }\end{array}$ & Phone: & +64044635281 \\
\hline mail: & tongnasoua@myvuw.ac.nz & Email: & John.overton@vuw.ac.nz \\
\hline
\end{tabular}

\section{Human Ethics Committee information}

If you have any concerns about the ethical conduct of the research you may contact the Victoria University HEC Convenor: Dr. Judith Loveridge. Email: hec@vuw.ac.nz or phone: +6444636028. 


\title{
Appendix 1.4. Information sheet for focus groups - Village Bank members
}

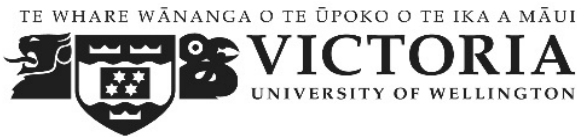

\author{
Research Understanding the influence of cultural factors on the perceptions of \\ title: empowerment and the use of microfinance for empowerment of \\ ethnic women in northern Laos. \\ Researcher: $\quad$ Souasong Tongnamavong \\ Supervisor: Prof. John Overton
}

Sabaidee and thank you for your interest in this research project. Please read the information below to gain a better understanding of the project. After reading this paper, please feel free to decide whether you would like to participate in this project or not. If you decide to participate, thank you so much for your time and contribution. If you decide not to participate, thank you for your consideration of my request and I wish you all the best with your studies or work.

\section{Who am I?}

My name is Souasong Tongnamavong, a Master's student of Development Studies at Victoria University of Wellington, New Zealand. As part of the university's requirements for the completion of my studies, I am doing a research project titled: "Understanding the influence of cultural factors on the perceptions of empowerment and the use of microfinance for empowerment of ethnic women in northern Laos".

I have chosen this topic because I am passionate about exploring how ethnic women perceive empowerment differently and how their cultural backgrounds have an influence on their use of microfinance to empower themselves. I hope that the findings of this research will not only serve my academic interest, but will also inform development theories and practices.

This research project has been reviewed and approved by the Human Ethics Committee of the Victoria University of Wellington, New Zealand.

\section{What is the aim of this project?}

This research aims to:

- explore the perceptions of different ethnic women about empowerment;

- explore cultural factors that have an influence on women's use of microfinance for empowerment;

- find out challenges and opportunities in the use of microfinance among different ethnic women, and the gap that current gender-based development approaches in rural Laos need to fill.

\section{How will I proceed?}

If you agree to participate, you will be invited to take part in focus group discussions consisting of around 6 people, including yourself. The group discussions will consist of only people from your ethnic background and who are village bank members.

The discussions will take between 1 to 2 hours and you will be asked about: 
- Your ethnic background: ethnic group, gender relations in your culture, the roles of women and men in your family;

- Your perceptions towards empowerment;

- Your perspectives on how the microfinance scheme has changed your life and relations with your husband;

- Your views about the factors of the microfinance scheme that contribute to your success;

- Your views about any cultural factors that have influence on the use of microfinance;

- Your opinion about other challenges facing you in making use of the financial scheme;

- Your strategies to overcome those challenges.

You will be asked to answer the questions in the group as a whole as well as in individual. You will be invited to engage in some activities that will help you enjoy the discussions and have fun.

The time for discussions totally depend on your decision and the place for conducting the activity will be decided so it suits all participants involved. Please note that a safe and uninterrupted place is highly desired given that confidentiality of the focus group needs to be guaranteed. Thus, only invited participants will attend the focus group meetings. With your permission, I will make audio recording and take notes of our discussions. You can leave the discussions at any time or refuse to answer any question without giving a reason.

Please also note that, if you choose to participate in the focus group, you need to adhere to a confidentiality clause, meaning that you will not share what was said in the group with others.

\section{What will happen after focus group discussions?}

Your information will be confidential. All the records, discussions and notes from the focus group will be kept securely. I will transcribe and analyse the information. Only my supervisor and I will read the notes or transcripts of the discussions. If data transcribers are required, they will be asked to sign a consent form which means that your information will not be disclosed to any other third parties.

Your name will not be mentioned in any reports, unless otherwise agreed. The discussion transcripts, summaries, and recordings will be kept securely and destroyed after five years since the end of the research.

\section{What will the project produce?}

The final report (thesis) of this project will be published and held at Victoria University of Wellington library. I may also use the results of my research for conference presentations and academic reports. I will take care of your information and not identify you in any presentation or report.

\section{What if you would like to withdraw from taking part in the project?}

You can withdraw from the research up to four weeks after the interview. If you withdraw, the information you provided will be destroyed or returned to you.

If you accept this invitation, what are your rights as a research participant? 
You do not have to accept this invitation if you don't want to. If you decide to participate, you have the right to:

- choose not to answer any question;

- ask for the recorder to be turned off at any time during the discussions;

- withdraw from the study up to four weeks after the discussions;

- ask any questions about the study at any time;

- receive a copy of your discussion recording (if it is recorded);

- read over and make comments on a written summary of our discussion;

- agree on another name for me to use rather than your real name;

- be able to read any reports of this research by emailing the researcher to request a copy.

If you have any questions or problems, who can you contact?

If you have any questions, either now or in the future, please feel free to contact either:

\section{Student:}

Name:

Souasong Tongnamavong

Course:

Master

Supervisor:

Prof. John Overton
Studies
Facebook: Souasong Tongnamavong

\section{Your village head:}
In case you cannot contact me directly, your village head can help you contact me.

Course coordinator and lecturer of Development Studies
School: School of Geography, School: School of Geography, Environment, and Earth Environment, and Earth Sciences +640221294723 (New Zealand)

Email: $\quad$ tongnasoua@myvuw.ac.nz Email: $\quad \underline{\text { John.overton@vuw.ac.nz }}$

\section{Human Ethics Committee information}

If you have any concerns about the ethical conduct of the research you may contact the Victoria University HEC Convenor: Dr. Judith Loveridge. Email: hec@,vuw.ac.nz or phone: +64 44636028 . 
Research title: Understanding the influence of cultural factors on the perceptions of empowerment and the use of microfinance for empowerment of ethnic women in northern Laos.

Researcher: Souasong Tongnamavong

Supervisor: Prof. John Overton

\begin{tabular}{|c|c|}
\hline Categories/themes & Questions \\
\hline (i). Self-confidence & $\begin{array}{l}\text { 1. Are you doing what you believe in? Or are you } \\
\text { doing what other people expected you to do? } \\
\text { 2. How often do you do it? } \\
\text { 3. How would you rank yourself in doing what you } \\
\text { believe in on a scale of } 1-10 \text { ? } \\
\text { 4. How do you respond to/deal with criticism? } \\
\text { 5. How would you rank yourself for your ability in } \\
\text { responding to/dealing with criticism on a scale of } \\
1-10 \text { ? } \\
\text { 6. How do you deal with fear/challenges? } \\
\text { 7. How would you rank yourself in dealing with } \\
\text { fear/challenges on a scale of } 1-10 \text { ? } \\
\text { 8. Does your confidence increase after joining the } \\
\text { program? Why or why not? }\end{array}$ \\
\hline (ii). Self-esteem & $\begin{array}{l}\text { 9. Go through Rosenberg's (1965) self-esteem } \\
\text { scale test. } \\
\text { 10. Does the program help increase your self- } \\
\text { appreciation? Why or why not? }\end{array}$ \\
\hline (iii). Sense of agency & $\begin{array}{l}\text { 11. Do you have a say in family? } \\
\text { 12. Do your family members know what you want? } \\
\text { 13. Do your family and community members know } \\
\text { what you can do best? } \\
\text { 14. Is there anything changed in your household } \\
\text { and community because of your action? } \\
\text { 15. Do you think you can create your own ideas } \\
\text { and strategies to improve your own and others' } \\
\text { situation, and cope with difficulties? } \\
\text { 16. Do you believe that you can learn new things } \\
\text { and be successful despite difficulties? } \\
\text { 17. Do you think you can help others, and others } \\
\text { can help you to be successful? } \\
\text { 18. Do you know who to ask for help with your } \\
\text { work? } \\
\text { 19. Does the program increase your sense of } \\
\text { agency? Why or why not? }\end{array}$ \\
\hline
\end{tabular}




\begin{tabular}{|c|c|}
\hline $\begin{array}{l}\text { (iv). Sense of 'self' in wider } \\
\text { context }\end{array}$ & $\begin{array}{l}\text { 20. Can you think about any things that come to } \\
\text { your mind when you think about yourself? } \\
21 \text {. Can you remember any information related to } \\
\text { yourself? } \\
\text { 22. Does the program make you change the } \\
\text { information about yourself? Why or why not? }\end{array}$ \\
\hline (v). Dignity & $\begin{array}{l}\text { 23. Do other people listen to you? } \\
\text { 24. Are you engaged in conversations? } \\
\text { 25. Do you think other people trust you for your } \\
\text { wisdom? } \\
\text { 26. Do you think people are not judgemental about } \\
\text { you, and respect you for who you are? } \\
27 \text {. Does the program have an effect on how people } \\
\text { behave toward you? Why or why not? }\end{array}$ \\
\hline $\begin{array}{l}\text { (vi). Views of cultural factors } \\
\text { in women's use of } \\
\text { microfinance for empowerment }\end{array}$ & $\begin{array}{l}\text { 28. What ethnic group or culture are you from? } \\
29 . \text { What are the basic gender relations in your } \\
\text { culture? } \\
\text { 30. What are women normally expected to do in } \\
\text { your family? } \\
\text { 31. What are men's roles in your family? } \\
\text { 32. Do you think most of the women from your } \\
\text { culture perceive gender relations in a similar way? } \\
\text { 33. Do you think that gender roles in your culture } \\
\text { are fair for both sides, men and women? Why or } \\
\text { why not? } \\
\text { 34. Do you think your culture has a significant } \\
\text { influence on your making use of your participation } \\
\text { in the financial scheme for empowering yourself? } \\
\text { If so, how? }\end{array}$ \\
\hline $\begin{array}{l}\text { (vii). Challenges facing women } \\
\text { in achieving empowerment }\end{array}$ & $\begin{array}{l}\text { 35. What are the challenges that you are facing in } \\
\text { making use of the financial scheme for achieving } \\
\text { your goals? } \\
\text { 36. What do you think are the biggest challenges } \\
\text { preventing you from achieving your goals? Why? }\end{array}$ \\
\hline $\begin{array}{l}\text { (viii). Strategies to overcome } \\
\text { challenges }\end{array}$ & $\begin{array}{l}\text { 37. What is your strategy to overcome the } \\
\text { challenges? } \\
\text { 38. What you hope your family, community, } \\
\text { development project, authority as well as the } \\
\text { government will do to help you achieve your goals? }\end{array}$ \\
\hline
\end{tabular}




\section{Appendix 2.2. Morris Rosenberg's Self-Esteem Scale}

Instructions:

Below is a list of statements dealing with your general feelings about yourself. Please indicate how strongly you agree or disagree with each statement.

1. On the whole, I am satisfied with myself.
Strongly Agree
Agree
Disagree
Strongly disagree

2. At times, I think I am not good at all.

$\begin{array}{llll}\text { Strongly Agree } & \text { Agree } & \text { Disagree } & \text { Strongly disagree }\end{array}$

3. I feel that I have a number of good qualities.

Strongly Agree Agree Disagree $\quad$ Strongly disagree

4. I am able to do things as well as most other people.
Strongly Agree
Agree
Disagree
Strongly disagree

5. I feel I do not have much to be proud of.
Strongly Agree
Agree
Disagree
Strongly disagree

6. I certainly feel useless at times.

$\begin{array}{llll}\text { Strongly Agree } & \text { Agree } & \text { Disagree } & \text { Strongly disagree }\end{array}$

7. I feel that I am a person of worth, at least on an equal plane with others.
Strongly Agree
Agree
Disagree
Strongly disagree

8. I wish I could have more respect for myself.
Strongly Agree
Agree
Disagree
Strongly disagree

9. All in all, I am inclined to feel that I am a failure.

$\begin{array}{llll}\text { Strongly Agree } & \text { Agree } & \text { Disagree } & \text { Strongly disagree }\end{array}$

10. I take a positive attitude toward myself.
Strongly Agree
Agree
Disagree
Strongly disagree 


\begin{tabular}{|c|c|}
\hline Categories/themes & Questions \\
\hline $\begin{array}{l}\text { (i). The influence of culture on } \\
\text { women's perceptions of } \\
\text { empowerment and aspirations } \\
\text { for empowerment }\end{array}$ & $\begin{array}{l}\text { 1. What do you know about ethnic women's } \\
\text { perceptions of empowerment and aspirations for } \\
\text { empowerment? } \\
\text { 2. What do you think are the influential factors on } \\
\text { their empowerment perceptions and aspirations? } \\
\text { 3. Do you think the culture they belong to } \\
\text { influences their perceptions of empowerment and } \\
\text { aspirations for empowerment? } \\
\text { 4. What do you consider as factors that help them } \\
\text { benefit the most from your project? } \\
\text { 5. Do you think their perceptions of empowerment } \\
\text { and aspirations for empowerment have changed } \\
\text { over time? If so, how? } \\
\text { 6. What could potentially be the factors influencing } \\
\text { these changes? }\end{array}$ \\
\hline $\begin{array}{l}\text { (ii). Role of GIZ in influencing } \\
\text { and creating conditions for the } \\
\text { empowerment of women }\end{array}$ & $\begin{array}{l}\text { 7. How do you help ethnic women to achieve } \\
\text { empowerment? } \\
\text { 8. What does your project provide or do to } \\
\text { encourage women's participation in the project, and } \\
\text { increase confidence, capacity and the ability to } \\
\text { control their lives? }\end{array}$ \\
\hline $\begin{array}{l}\text { (iii). Women's networking, } \\
\text { socialising, participation and } \\
\text { engagement experiences, and } \\
\text { motivations in the project }\end{array}$ & $\begin{array}{l}\text { 9. What is the working and supporting environment } \\
\text { like in the village bank? } \\
10 \text {. What do village bank members like to do the } \\
\text { most when they come to use the services? } \\
\text { 11. Is there any observable different interests } \\
\text { among ethnic women in participating in the } \\
\text { project? } \\
12 \text {. What factors influence these women's } \\
\text { motivation, participation, and engagement in the } \\
\text { project? } \\
13 \text {. What difficulties do you think ethnic women } \\
\text { experience in the program? } \\
14 \text {. What difficulties do ethnic women experience } \\
\text { in their daily lives? } \\
\text { 15. As a development agency targeting rural } \\
\text { women, what is your role in helping them } \\
\text { overcome these difficulties? }\end{array}$ \\
\hline $\begin{array}{l}\text { (iv). Aspirations for } \\
\text { empowerment }\end{array}$ & $\begin{array}{l}\text { 16. What do you think would be an effective way to } \\
\text { help ethnic women achieve empowerment, } \\
\text { reduce women's poverty and improve gender } \\
\text { inequality? } \\
\text { 17. Is there anything else you would like to } \\
\text { comment on? }\end{array}$ \\
\hline
\end{tabular}


Appendix 2.4. Interview questions for officers of Bank of Laos

\begin{tabular}{|l|l|}
\hline Categories/themes & Questions \\
\hline (i). Perception towards & $\begin{array}{l}\text { 1. What is your view towards the role of } \\
\text { microfinance in poverty reduction in Laos? } \\
\text { 2. Do you think microfinance can help reduce } \\
\text { gender inequality in Laos? If so, how? }\end{array}$ \\
\hline $\begin{array}{l}\text { (ii). The influence of culture on } \\
\text { empowerment perceptions }\end{array}$ & $\begin{array}{l}\text { 3. Do you think culture has an influence on the } \\
\text { outcome of using microfinance among women in } \\
\text { Laos? } \\
\text { 4. Do you think the culture one woman belongs to } \\
\text { has an influence on her perception of } \\
\text { empowerment? }\end{array}$ \\
$\begin{array}{l}\text { 5. What do you think could be another factor } \\
\text { influencing women's perceptions of } \\
\text { empowerment? }\end{array}$ \\
\hline $\begin{array}{l}\text { (iii). The influence of culture } \\
\text { on the use of microfinance for } \\
\text { empowerment }\end{array}$ & $\begin{array}{l}\text { of microfinance to empower themselves? If so, } \\
\text { how? } \\
\text { 7. What do you think are other burdens and barriers } \\
\text { preventing ethnic women from being able to utilise } \\
\text { the financial scheme to empower themselves? }\end{array}$ \\
\hline $\begin{array}{l}\text { (iv). The institutional strategies } \\
\text { options for empowering } \\
\text { and interventions }\end{array}$ & $\begin{array}{l}\text { institution using to shape the perceptions of the } \\
\text { women and ensure they can benefit from the } \\
\text { financial scheme? } \\
\text { 9. What are the strengths and challenges in using } \\
\text { these strategies and interventions? } \\
\text { 10. How have these strategies and interventions } \\
\text { helped ethnic women to achieve empowerment? }\end{array}$ \\
$\begin{array}{l}\text { 11. What are your lessons learned in helping ethnic } \\
\text { women achieve self-empowerment? } \\
\text { 12. What are the opportunities or options to } \\
\text { effectively help ethnic women achieve } \\
\text { empowerment? } \\
\text { 13. Is there anything else you would like to add or } \\
\text { comment on? }\end{array}$ \\
\hline
\end{tabular}




\section{Activities}

"Conducting empowerment perceptions and aspirations exercise - the Stepping Stones diagram approach" - 30 minutes

- The participants will be in one group of six people from the same ethnic background.

- In front of them will be a large piece of paper with a drawing of a river as a metaphor for the women's path and struggle to achieve empowerment.

- The participants will be asked to think about what power they have now (e.g. decision-making, self-autonomy, and control over their assets) and what kinds of power they want to achieve in the future. Then they will be asked to brainstorm what is needed to help them achieve their empowerment aspirations.

- Participants' ideas of power, aspirations and enablers will be written down on small notes then these notes will be laid on their proper positions on the metaphoric river.

- Then the group will turn to a group discussion which will be facilitated by the researcher. Note that the researcher will encourage the participants to lead the discussion themselves rather than being led by the researcher.

\section{Discussions and sample questions}

\subsection{Cultural background}

2.1.1. Do men and women have different roles and do different work in your family? 2.1.2. If so, what do you think could be the reasons for the division of roles between men and women?

2.1.3. What is your image of yourself in your family?

2.1.4. How do you feel about your image?

\subsection{Empowerment perceptions and aspirations}

2.2.1. What do you think empowerment would mean to you?

2.2.2. What are your expectations from participating in the financial scheme?

2.2.3. Do you feel like you have some power? If so, what are they and how have you got that power? If not, why not?

2.2.4. What benefits do you have from participating in the village bank?

2.2.5. Has the program changed your view of personal empowerment? If so, how?

2.2.6. Do those benefits help you empower yourself? If so, how? If not, why not?

2.2.7. What do you see yourself as on the other side of the river?

2.2.8. What means do you need to help you get there?

2.2.9. What are the barriers preventing you from reaching the other side of the river?

2.2.10. Do you think culture has a significant influence on your making use of the program? If so, how?

2.2.11. Do you think that such barriers can be removed or overcome? If so, by what means?

2.2.12. Do you have anything else to add or comment on? 
Appendix 2.6. Stepping stones diagram

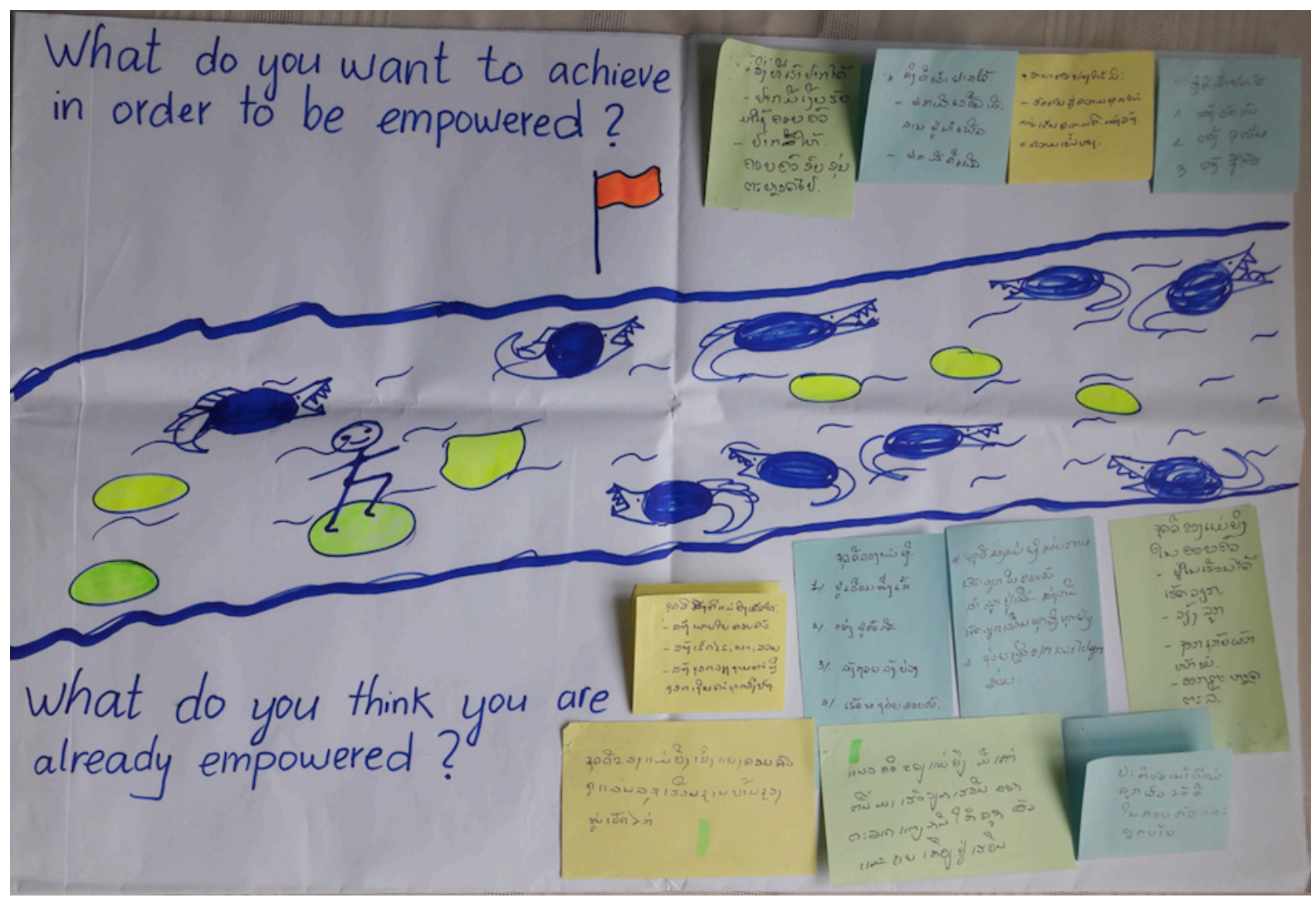




\section{Appendix 3.1. Participant consent form - Interviews with Village Bank members}

TE WHARE WĀNANGA O TE ŪPOKO O TE IKA A MĀUI

59

* $\because$ university of wellington

Research Understanding the influence of cultural factors on the perceptions of title: $\quad$ empowerment and the use of microfinance for empowerment of ethnic women in northern Laos.

Researcher: Souasong Tongnamavong

Supervisor: Prof. John Overton

I have read the 'Participant Consent Form' or the form has been explained to me. The researcher has also explained to me about the process of interview. I understand that I can stop the interview at any time without giving any reasons.

By signing below, I acknowledge that:

- My identity will be kept confidential so my real name will not be mentioned in any reports, unless otherwise agreed;

- I may withdraw from this study up to four weeks after the interview, and any information that I have provided will be returned to me or destroyed;

- The information I have provided will be destroyed five years after the research ends;

- I permit my input and any information I provide to be interpreted, analysed, and published in student reports and in a thesis, as well as to be presented at conferences and to be available in other forms of publication such as journal articles and magazine or newspaper articles;

- The accompanying person, if any, including my husband, will keep confidentiality of the interview.

Please check where is applied:

I permit the researcher to make audio recordings.

I permit the researcher to use my quotes in the research.

I request a summary of my interview.

I request to receive a summary of the report via my address below

I, , agree and consent to the above statements.

Signature:

Date:

Position:

Institution:

Mobile:

E-mail/Facebook:

Name of accompanying person 1 : , Signature:

Name of accompanying person 2: Signature: 


\section{Appendix 3.2. Participant consent form - Interviews with institutional officers}

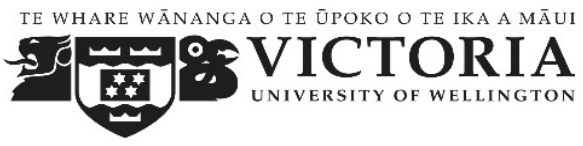

Research Understanding the influence of cultural factors on the perceptions of title: empowerment and the use of microfinance for empowerment of ethnic women in northern Laos.

Researcher: $\quad$ Souasong Tongnamavong

Supervisor: Prof. John Overton

I have read the 'Participant Consent Form' or the form has been explained to me. The researcher has also explained to me about the process of interview. I understand that I can stop the interview at any time without giving any reasons.

By signing below, I acknowledge that:

- My identity will be kept confidential so my real name will not be mentioned in any reports, unless otherwise agreed;

- I may withdraw from this study up to four weeks after the interview, and any information that I have provided will be returned to me or destroyed;

- The information I have provided will be destroyed five years after the research ends;

- I permit my input and any information I provide to be interpreted, analysed, and published in student reports and in a thesis, as well as to be presented at conferences and to be available in other forms of publication such as journal articles and magazine or newspaper articles;

Please check where is applied:

I permit the researcher to make audio recordings.

I permit the researcher to use my quotes in the research.

I request a summary of my interview.

I request to receive a summary of the report via my address below

$\mathrm{I}$, , agree and consent to the above statements.

Signature:

Position:

Mobile:
Date:

Institution:

E-mail/Facebook: 


\section{Appendix 3.3. Participant consent form - Focus groups with Village Bank members}

TE WHARE WĀNANGA O TE ŪPOKO O TE IKA A MĀUI

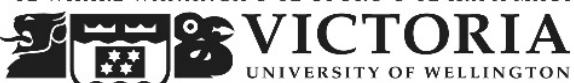

$\begin{array}{ll}\begin{array}{l}\text { Research } \\ \text { title: }\end{array} & \begin{array}{l}\text { Understanding the influence of cultural factors on the perceptions of } \\ \text { empowerment and the use of microfinance for empowerment of } \\ \text { ethnic women in northern Laos. }\end{array} \\ \text { Researcher: } & \text { Souasong Tongnamavong } \\ \text { Supervisor: } & \text { Prof. John Overton }\end{array}$

I have read the 'Participant Consent Form' or the form has been explained to me. The researcher has also explained to me about the process of conducting focus groups. I understand that I can stop getting involved in the discussions at any time without giving any reasons.

By signing below, I acknowledge that:

- My identity will be kept confidential so my real name will not be mentioned in any reports, unless otherwise agreed;

- All the information shared during the focus group should remain confidential. I therefore agree not to share or comment with anyone else what participants said in the discussions;

- I may withdraw from this study up to four weeks after the group discussions, and any information that I have provided will be returned to me or destroyed;

- In case of withdrawal during the discussion, the participant's comments cannot be retracted;

- The information I have provided will be destroyed five years after the research ends;

- I permit my input and any information I provide to be interpreted, analysed, and published in student reports and in a thesis, as well as to be presented at conferences and to be available in other forms of publication such as journal articles and magazine or newspaper articles.

Please check where is applied:

I agree the focus groups being audio recorded.

I agree to some of statements being quoted in the research.

I acknowledge the confidentiality of our focus group discussions and that what we discuss will be kept among our group members.

I request to receive a summary of the report via my address below

$\mathrm{I}$, , agree and consent to the above statements.

Signature:

Date:

Position:

Institution:

Mobile:

E-mail/Facebook: 
Appendix 4. Human ethics approval

TE WHARE WĀNANGa o TE ŪPOKO O TE IKa A MĀUI

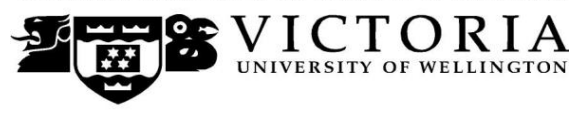

MEMORANDUM

\begin{tabular}{l|l}
\hline TO & Song Tongnamavong \\
\hline FROM & Dr Judith Loveridge, Convenor, Human Ethics Committee \\
\hline DATE & 20 May 2018 \\
\hline PAGES & 1 \\
\hline SUBJECT & $\begin{array}{l}\text { Ethics Approval: } 25990 \\
\text { Understanding the influence of cultural factors on the perceptions } \\
\text { of empowerment and the use of microfinance for empowerment of } \\
\text { women in northern Laos }\end{array}$ \\
\hline
\end{tabular}

Thank you for your application for ethical approval, which has now been considered by the Human Ethics Committee.

Your application has been approved from the above date and this approval is valid for three years. If your data collection is not completed by this date you should apply to the Human Ethics Committee for an extension to this approval.

Best wishes with the research.

Kind regards

Judith Loveridge

Convenor, Victoria University Human Ethics Committee 


\section{Appendix 5. Support letter from supervisor}

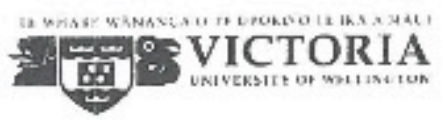

School of Geopraphy, Environment and Earth Sciences Victoria University of Wellington,

Wellington 6012 New Zealand

Email: johnoverton@vuw,ac.nz

Phone: $+64 \$ 4635281$

22 May 2018

\section{To Whom It May Concern}

I am writing this letter as the supervisor of Souasong Tongnamavong to support him in collecting data for his master's thesis in Development Studies.

OSouasong was awarded New Zealand ASEAN Scholarship to study a Masters of Development Studies at School of Geography, Environment and Earth Sciences, Victoria University of Wellington, New Zealand. In order to complete his degree, he needs to conduct research and write a thesis based on the fieldwork in his home country during 2018.

Souasong proposes to conduct research on "understanding the influence of cultural factors on the perceptions of empowerment and the use of microfinance for empowerment of ethnic women in northern Laos". His field research will be conducted in Luangnamtha, Laos, from 01 June to 20 August 2018.

During his fieldwork, 1 would be most grateful if you could assist him in providing relevant information and any other support to complete his research.

If you require any further information please contact me on +6444635281 or by email at johnoverton@vuw.ac.nz

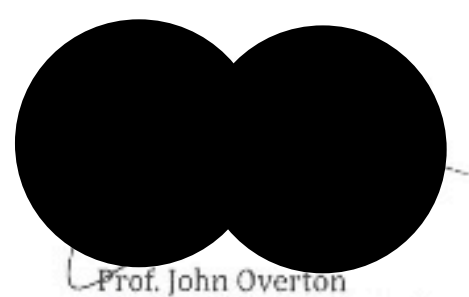

Director, Postgraduate Programme in Development Studies

School of Geography, Environment, and Earth Sciences

Victoria University of Wellington, New Zealand 


\section{Appendix 6.1. Request letter to Bank of Laos}

Request Letter to Bank of the Lao PDR - Data Collection with Access to Finance for the Poor (AFP) project

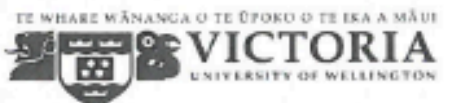

Dear: Director General of Financial Institution Supervision Department

About: Data collection with Access to Finance for the Poor (AFP) project for the completion of a master's thesis

I am Souasong Tongnamavong, a Lao student studying a Master of Development Studies degree at School of Geography, Environment and Earth Sciences at Victoria University of Wellington, New Zealand (VUW) under New Zealand Scholarship. As part of the University's requirements for the completion of my study, I am doing a research project titled: "Understanding the influence of cultural factors on the perceptions of empowerment and the use of microfinance for empowerment of ethnic women in northern Laos", by taking Access to Finance for the Poor (AFP) project as a case study. The data collection process, which has been approved by the University, will be conducted during June and August 2018. Then, the collected data will be used to write a master's thesis, which is expected to finish by February 2019. Details of the research are attached to this request letter.

The data collection will take place in some villages in Luangnamtha province where the AFP project is operating. There will be face-to-face interviews and focus group discussions conducted with different ethnic women participating in AFP project. Their identities, participation and information will be kept confidential.

Therefore, with this letter I hope that your permission will be given as it is very necessary for the completion of my data collection process for the research.

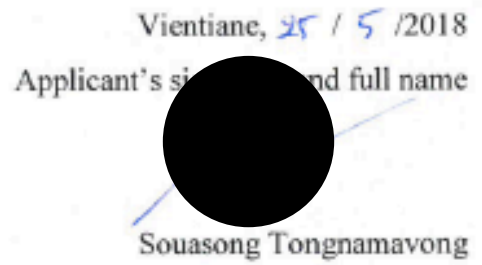

Attached documents:

Support letter from Victoria University of Wellington, New Zealand (Lao and English versions)

A summary of research proposal (Lao version)

Full research proposal (English version) 


\title{
Appendix 6.2. Request letter to GIZ Laos
}

\author{
Request Letter to (rIZ - Data Collection with Access to Finance for the Poor (AFP) \\ project

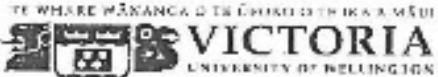 \\ Dear: Chairman of GIZ Laos \\ About: Data coliection with Access to Finance for the Poor (AFP) project \\ for the cumpletion of a master's thesis
}

I am Souasong Tongnamavong, a Lao stutent studying a Master of Dovclopment Studies degree at School of Geography, Environment and Earth Sciences at Victoria University of Wellington, New Zealand (VUW) under New Zealand Scholarship. As part of the University's requirements for the completim of my study, 1 am doing a research project titled: "Understanding the influence of cultural foctors on the perceptions of empowerment and the nse of microfinance for emprowerment of ethic women in northern Laos", by taking Access to Finance for the Poor (AFP) project as a case study. The data collection process, which has been approved by the University, will be conducted during June and August 2018. Then the collected data will be used to write a master's thesis, which is expecied $t o$ be finished by February 2019. Details of the research are attached to this request letter.

The data collection will take place in some villages in Namtha district, Luangnamtha province where the AFP project is operating. There will be face-to-face interviews and locus group discussions conducted with different ethric women participating in AFP project. Their identities, participation and information will be kept confidential. Upon your approval of this request lelter, the participation of the villagers and any of your staff membersin this studywill not lead to any losses or disadvantages of their rights and cmployment in any way.

For the purpose of sample selcction, I would like to have your cooperation as follows

1). Directing me to a particular village that consists of thee ethnic groups: Lao, Khmu, and Hmong in which women are village bank members; or if there is no such village, please direct me to thee different villages in which each one has an ethnic group:

2). Introducing me to village heads;

3). Providing me with a list of village bank members in order to facilitate my sumplc selection process. 
Therefore, with this letter I hope that your permission will be given, and your cooperation will be provided as they are very necessary for the completion of my data collection process for the research

Vientiane, $3 / 6 / 2018$

Name and signature of applicant:

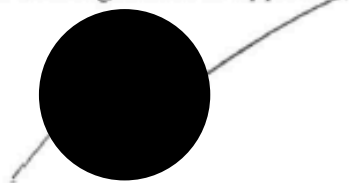

Souasong Tongnamavong

Attached documents:

- Support letter from Vietoria University of Wellington, New Zealand (Lao and English versions)

A summary of research proposal (Lao version)

Full research proposal (English version)

- Research permission letter from Bank of the Lao PDR 


\section{Appendix 6.3. Approval letter from Bank of Laos - Financial Institutions Supervision Department (FISD-BoL)}

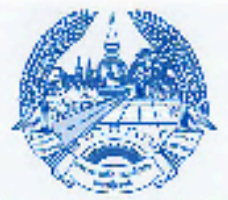

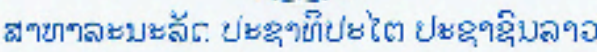

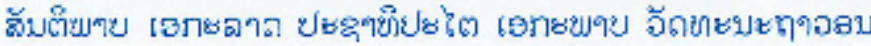

ภะบาถาบเม่าต สปป วขอ

กิมถุ๊บคองตะฤาขับภาบเวิม
เลกง์ 71 /ภถม

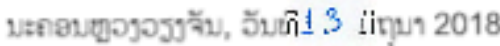

$$
\text { ขับริเสจ้วายบ }
$$

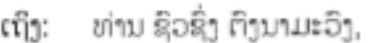

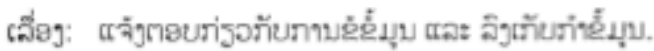

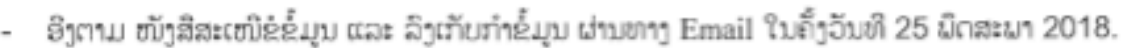

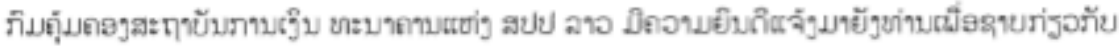

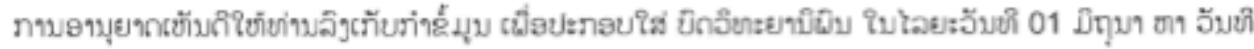

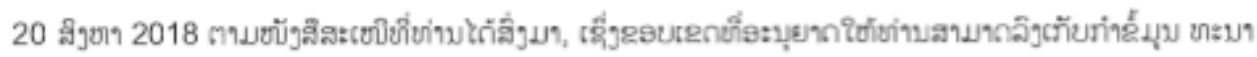

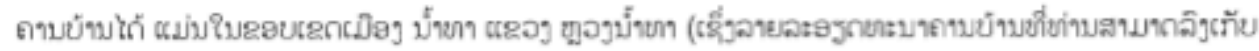

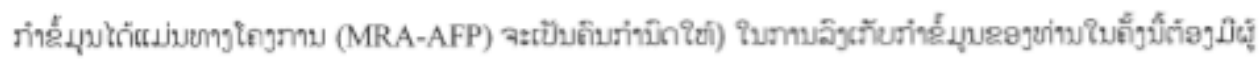

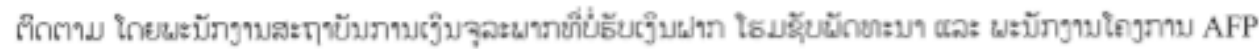

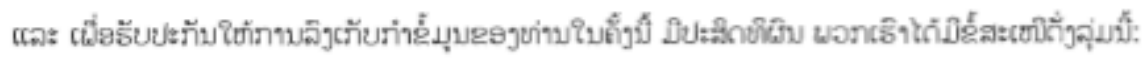

* घี่สะเขึ:

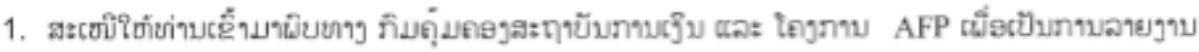

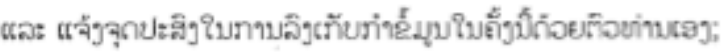

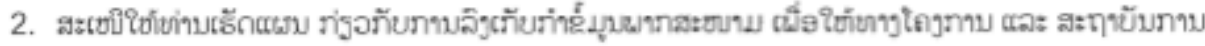

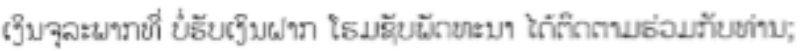

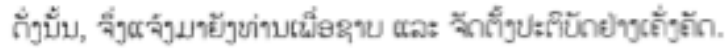

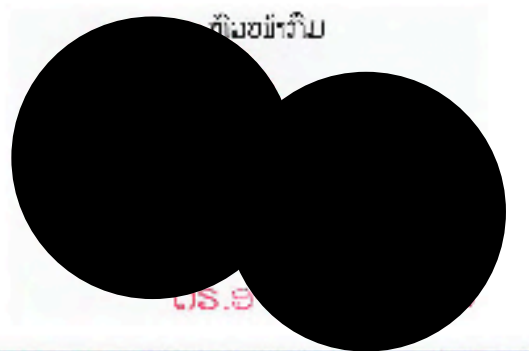

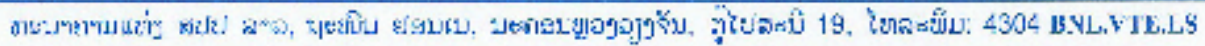

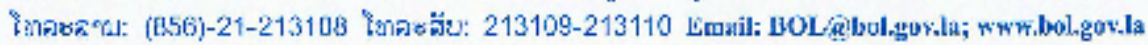


Appendix 6.4. Request letter from BoL to GIZ

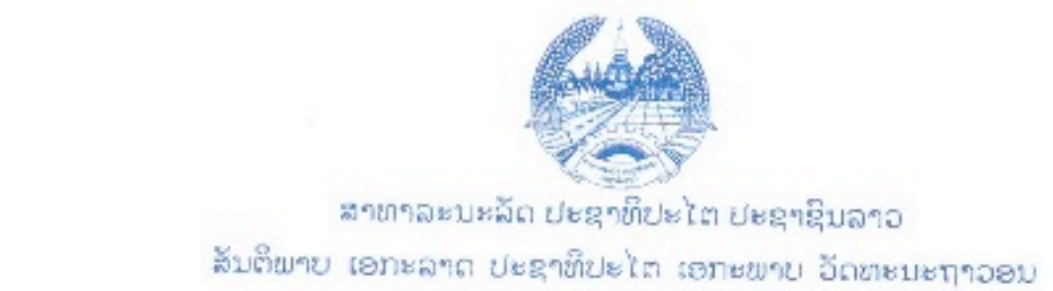

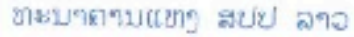

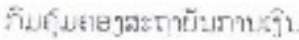

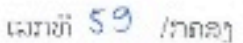

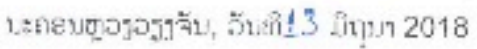

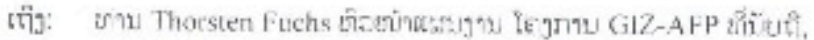

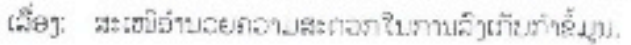

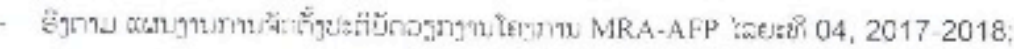

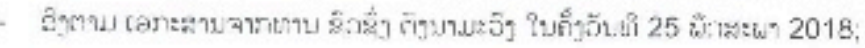

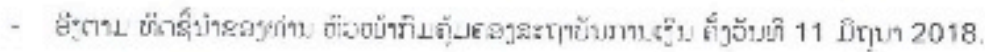

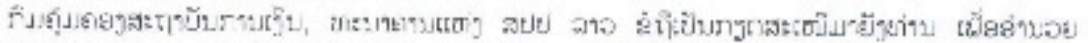

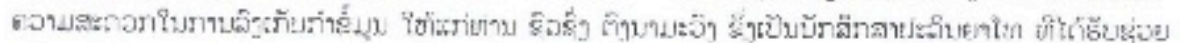

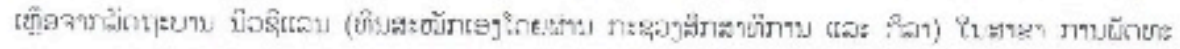

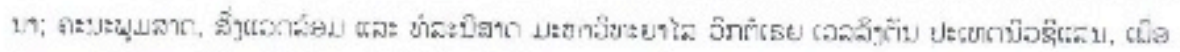

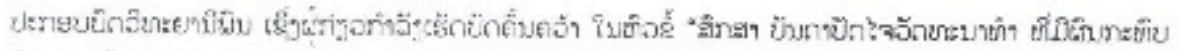

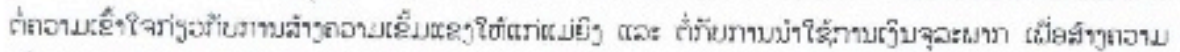

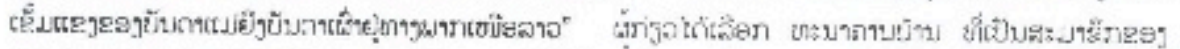

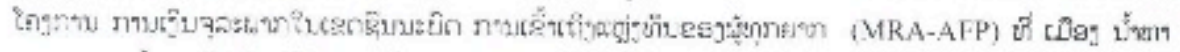

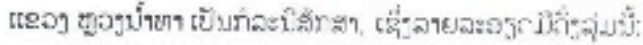

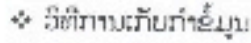

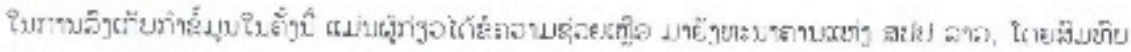

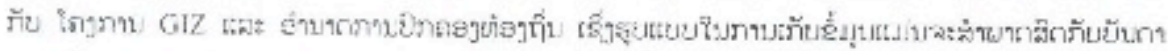

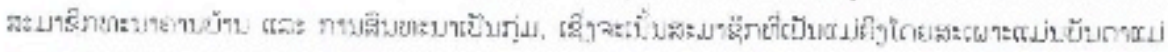

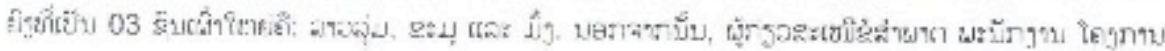

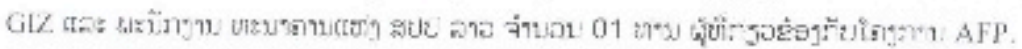

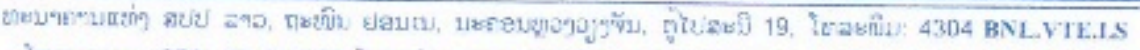

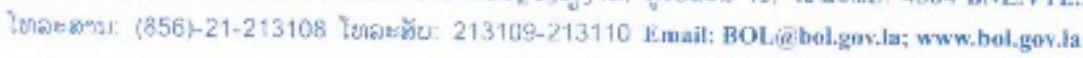




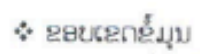

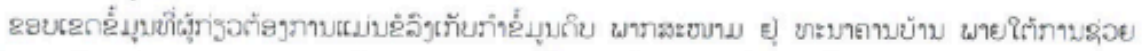

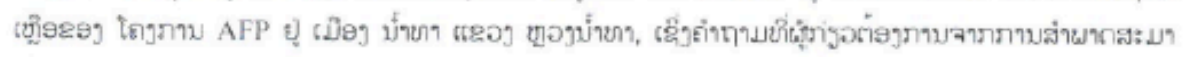

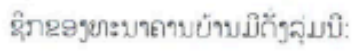

ถำทาบธูตรา:

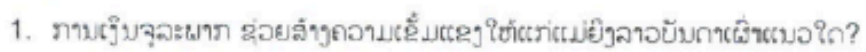
ถำฤนย่อย:

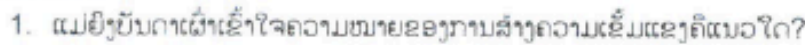

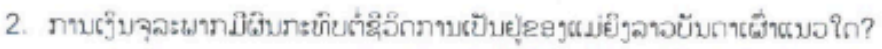

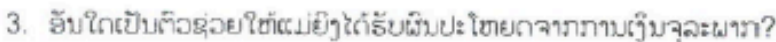

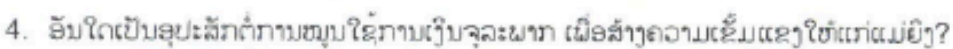

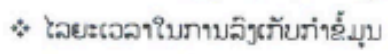

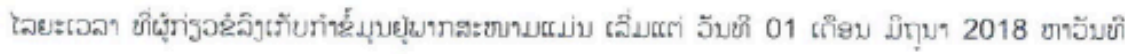
20 ลิวตา 2018.

* อั้สะเขึ:

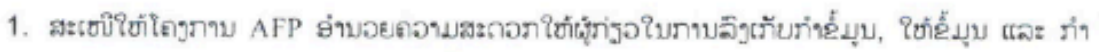

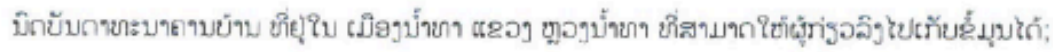

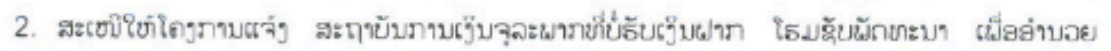
ถอามสะกองใช้เสู่รับอก้อย.

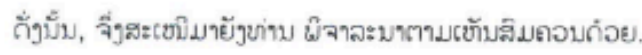

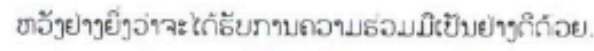

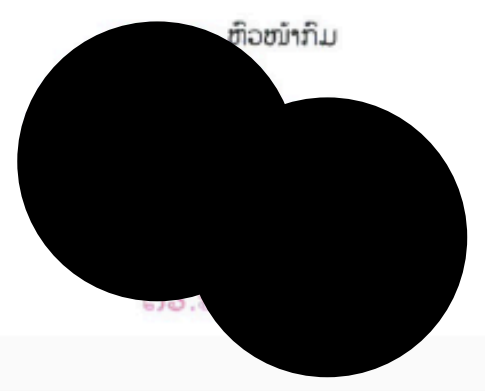




\section{Appendix 6.5. Support letter from GIZ Laos - The AFP project}

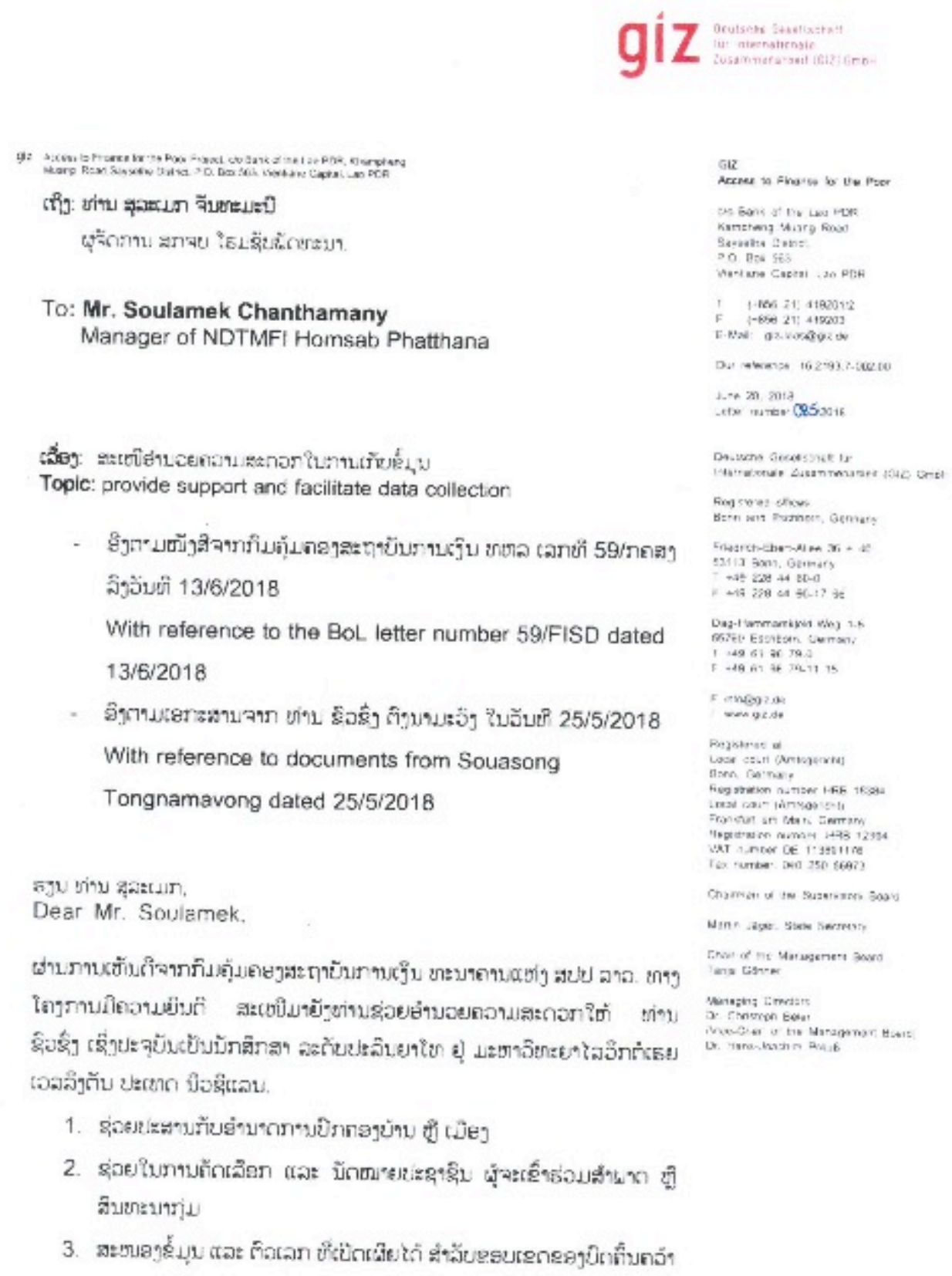

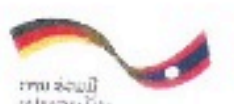

tsispation 


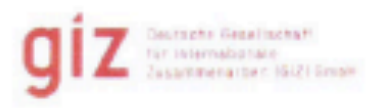

$\sin$

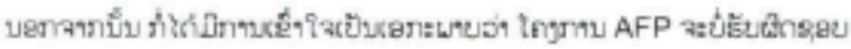

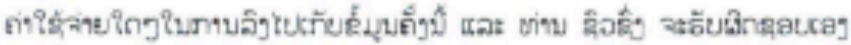
ย่างเนี้มจ่อบ

With no objection from the Financial Supervision Department, Bank of the Lao PDR. We would like to kindly ask you to support Mr. Souasong, a Lao student who is currently undertaking master degree at Victoria University of Wellington. New Zealand.

1. Support in coordinating with village/district authorities

2. Support in selecting and making appointment with participants for interview and group discussion

3. Provide accessible information and data for the scope of research

It was mutually understood that AFP will not be heid responsible for any costs incurred in this visit and Souasong shall take full responsibility for himself.

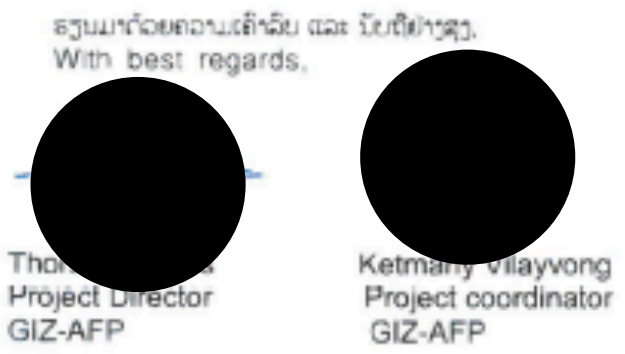

